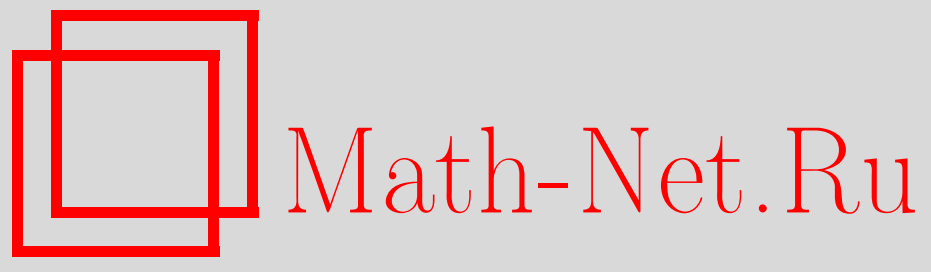

В. Б. Шехтман, Квадраты модальных логик с дополнительными связками, УМH, 2012, том 67, выпуск 4, 129186

DOI: https://doi.org/10.4213/rm9442

Использование Общероссийского математического портала Math-Net.Ru подразумевает, что вы прочитали и согласны с пользовательским соглашением http://www .mathnet.ru/rus/agreement

Параметры загрузки:

IP: 54.92 .164 .108

26 апреля 2023 г., 09:51:19

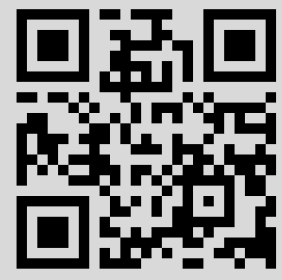


УДК 510.6

\section{Квадраты модальных логик \\ с дополнительными связками}

\section{В. Б. Шехтман}

В работе исследуются двумерные модальные логики специального вида - "квадраты Сегерберга". Они определяются как обычные квадраты модальных логик с дополнительными связками, которые соответствуют симметрии относительно диагонали и двум проекциям на нее. Для этих логик во многих случаях строится конечная аксиоматика, доказывается полнота и финитная аппроксимируемость. Строится перевод квадратов Сегерберга в классическую логику предикатов.

Библиография: 21 название.

Ключевые слова: модальная логика, произведение модальных логик, квадрат Сегерберга, полнота, финитная аппроксимируемость, метод фильтраций, классическая логика предикатов, проблема разрешения.

\section{СОДЕРЖАНИЕ}

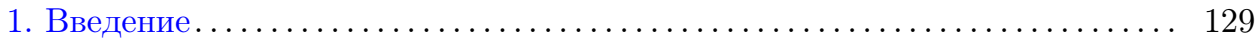

2. Модальные логики и их произведения .......................... 131

3. Квадраты Сегерберга ................................. 140

4. Аксиоматизация квадратов Сегерберга ......................... 144

5. О квадратах деревьев ................................. 163

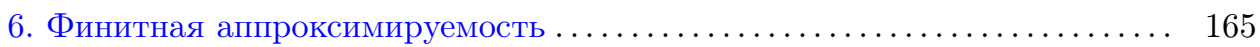

7. Финитная аппроксимируемость квадратами ................... 173

8. Погружение в классическую логику предикатов ................. 183

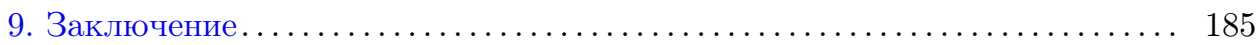

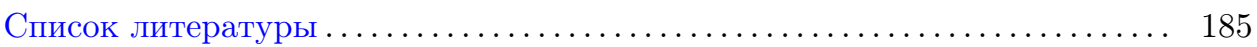

\section{1. Введение}

Модальные логики можно условно разделить на два вида: мономодальные и полимодальные. Хотя оба вида логик исследуются похожими методами, в полученных до сих пор результатах имеется отличие. А именно, в мономодальном

Работа выполнена при поддержке РФФИ (гранты № 11-01-00281_а, № 11-01-00958_a, № 11-01-93107-НЦНИЛ_а, № 11-01-92471-МНТИ_а) и программы "Ведущие научные школы" (грант НШ-845.2008.1).

(C) В. Б. Шехтман, 2012 
случае доказан ряд положительных свойств для больших семейств, устойчивых относительно расширений (как например, теорема Файна о полноте всех транзитивных логик конечной ширины [1]). В полимодальном же случае исследование произвольных расширений конкретной логики часто оказывается слишком трудной задачей. Поэтому больше изучались полимодальные логики, построенные с помощью естественных операций - синтаксических или семантических. Одна из таких операций - декартово произведение модальных логик была определена в [2] и [3] и затем исследовалась рядом авторов - в том числе в книге [4] и работах [5]-[8]; см. также обзор [9].

По сравнению с известными "одномерными" модальными логиками (такими как $\mathbf{K}, \mathbf{S 4}, \mathbf{S 5})$, произведения обладают бо́льшим разнообразием свойств как положительных, так и отрицательных. Например, трехмерные произведения, как правило, неразрешимы и конечно неаксиоматизируемы [4]. Для двумерных произведений во многих случаях доказана разрешимость, финитная аппроксимируемость и конечная аксиоматизируемость. В то же время среди двумерных произведений имеются конечно аксиоматизируемые неразрешимые логики довольно простого вида [10] (для сравнения отметим, что все известные примеры одномерных логик с такими свойствами нетривиальны - см., например, [11], [12]).

Квадраты модальных логик - важный частный случай произведений. С одной стороны, квадраты полезны для изучения классической логики, поскольку они погружаются в классические теории первого порядка и эквациональные теории алгебр отношений. С другой стороны, произведение любых двух модальных логик погружается в квадрат их соединения, т. е. все произведения по существу сводятся к квадратам [6].

По определению, квадрат $n$-модальной логики является $2 n$-модальной логикой, модальности которой соответствуют $n$ "горизонтальным" и $n$ "вертикальным" отношениям достижимости на "квадратных" шкалах Крипке. Но на квадратных шкалах имеются и другие естественные отношения, которые приводят к новым модальностям. Эти модальности рассматривались еще в ранних работах о двумерных модальных логиках [13], [14], позднее - в [15]; совсем недавно были получены интересные результаты о квадратах модальных логик с выделенной диагональю [16]. Однако, в отличие от произведений, для “обогащенных квадратов" пока еще нет сколько-нибудь систематической теории.

Настоящая работа частично закрывает этот пробел. В ней изучаются квадраты модальных логик со связками Сегерберга [13], которые соответствуют симметрии относительно главной диагонали и двум проекциям на нее. Мы показываем, что во многих случаях свойства таких логик аналогичны свойствам обычных квадратов.

Основные результаты - теоремы о полноте и финитной аппроксимируемости - содержатся в разделах 4, 6 и 7. В разделе 4 доказано, что квадраты Сегерберга полных модальных логик хорновского типа получаются добавлением стандартных аксиом к обычным квадратам (которые в этом случае также аксиоматизируются стандартным образом - это было известно из предыдущих работ). Доказательство полноты проводится игровым методом, который развивает аналогичные методы для обычных квадратов и релятивизованных 
квадратов [4], [8]. Следует отметить, что, по недосмотру автора, доказательство из [8] не годится для обычных квадратов, но предложенная в настоящей работе модификация исправляет его.

В разделе 6 доказывается финитная аппроксимируемость (из которой следует разрешимость) для квадратов Сегерберга минимальных модальных логик. Доказательство основано на методе конечной глубины из работы [5], с некоторыми усовершенствованиями. В разделе 7 этот результат усиливается: доказана полнота тех же логик относительно квадратов Сегерберга конечных деревьев. Для построения контрмоделей здесь снова используется игровой метод в другом варианте, поскольку бесконечная игра из раздела 4 в данном случае неприменима. Идейно доказательство теоремы 7.2 близко к доказательствам аналогичных результатов для обычных произведений в [6] и [7], но использование игры делает его, на наш взгляд, более прозрачным. Отметим, что до сих пор игры в доказательствах подобных результатов (о финитной аппроксимируемости произведениями) не применялись.

В разделе 8 описывается связь квадратов Сегерберга с классической логикой предикатов и дается интерпретация полученных в работе результатов в терминах классической логики.

\section{2. Модальные логики и их произведения}

Напомним сначала некоторые известные определения и факты.

Мы рассматриваем $n$-модалъные формулы в пропозициональном языке $\mathscr{L}_{n}$ со счетным множеством пропозициональных переменных $\mathrm{PV}:=\left\{p_{1}, p_{2}, \ldots\right\}$, классическими связками $(\supset, \perp)$ и одноместными модальными связками $\square_{1}, \ldots, \square_{n}$; остальные связки $\left(\wedge, \vee, \neg, \top, \equiv, \diamond_{i}\right)$ - производные. Модальная формула, не содержащая переменных, называется замкнутой.

Для последовательности индексов $\alpha=\left(i_{1}, \ldots, i_{r}\right) \in\{1, \ldots, n\}^{r}$ обозначим

$$
\diamond_{\alpha}:=\diamond_{i_{1}} \ldots \diamond_{i_{r}}, \quad \square_{\alpha}:=\square_{i_{1}} \ldots \square_{i_{r}} .
$$

Если $\alpha-$ пустая последовательность, то $\diamond_{\alpha}, \square_{\alpha}-$ пустые слова.

$\mathscr{L}_{n}\lceil m$ обозначает множество всех $n$-модальных формул, построенных из конечного множества переменных $\mathrm{PV}\left\lceil m:=\left\{p_{1}, p_{2}, \ldots, p_{m}\right\}\right.$.

Длиной модальной формулы $A$ называется число вхождений в нее логических связок; обозначение: $|A|$.

(Нормальная) n-модальная логика - это множество $n$-модальных формул, содержащее все классические тавтологии, формулы

$$
\square_{i}\left(p_{1} \supset p_{2}\right) \supset\left(\square_{i} p_{1} \supset \square_{i} p_{2}\right),
$$

где $1 \leqslant i \leqslant n$, и замкнутое относительно правил подстановки, modus ponens и $\square$-введения $\left(A / \square_{i} A\right), 1 \leqslant i \leqslant n$.

Минимальная $n$-модальная логика обозначается через $\mathbf{K}_{n}(\mathbf{K}-$ при $n=1)$. Для $n$-модальной логики $\boldsymbol{\Lambda}$ и множества $n$-модальных формул $\Gamma$ наименьшая модальная логика, содержащая $\boldsymbol{\Lambda} \cup \Gamma$, обозначается через $\boldsymbol{\Lambda}+\Gamma(\boldsymbol{\Lambda}+A$ при $\Gamma=\{A\})$. Логики вида $\mathbf{K}_{n}+A$, где $A-n$-модальная формула, называются конечно аксиоматизируемыми. 
Множества вида $\boldsymbol{\Lambda}\left\lceil m:=\boldsymbol{\Lambda} \cap \mathscr{L}_{n}\lceil m\right.$, где $\boldsymbol{\Lambda}-n$-модальная логика, называются ограниченными п-модальными логиками.

ОПРЕДЕЛЕНИЕ 2.1. Соединением (англ. - fusion) $n$-модальной логики $\mathbf{L}_{1}$ и $m$-модальной логики $\mathbf{L}_{2}$ называется $(n+m)$-модальная логика

$$
\mathbf{L}_{1} * \mathbf{L}_{2}:=\mathbf{K}_{n+m}+\mathbf{L}_{1}+\mathbf{L}_{2}^{+n}
$$

где $\mathbf{L}_{2}^{+n}$ получается из $\mathbf{L}_{2}$ переименованием всех модальностей $\square_{j}$ в $\square_{j+n}$.

Для построения семантики $n$-модальных формул используются $n$-шкаль Крипке, т. е. непустые множества ("множества миров") с $n$ бинарными отношениями: $\left(W, R_{1}, \ldots, R_{n}\right)$. Тройки $(x, y, i)$, где $x R_{i} y$, называются стрелками в $F$. (Для удобства мы будем также говорить, что $x R_{i} y$ - стрелка.) Задав на шкале $F=\left(W, R_{1}, \ldots, R_{n}\right)$ оценку пропозициональных переменных $\theta: \mathrm{PV} \longrightarrow \mathscr{P}(W)$, получаем модель Крипке $(F, \theta)$. Истинность модальной формулы в точке $w$ модели Крипке $M$ (обозначение: $M, w \vDash A$ или $w \vDash A$ ) определяется индукцией по длине стандартным образом:

$$
\begin{gathered}
w \nvdash \models \perp ; \quad w \vDash p_{i} \Longleftrightarrow w \in \theta\left(p_{i}\right) ; \quad w \vDash A \supset B \Longleftrightarrow(w \not \models A \text { или } w \vDash B) ; \\
w \vDash \square_{i} A \Longleftrightarrow \forall u\left(w R_{i} u \Rightarrow u \vDash A\right) .
\end{gathered}
$$

Формула $A$ истинна в модели Крипке $M$ (обозначение: $M \vDash A$ ), если она истинна во всех ее точках. Формула $A$ общезначима в шкале $F$ (обозначение: $F \vDash A$ ), если она истинна в любой модели Крипке на $F$. Формула $A$ общезначима в точке $x$ шкалы $F$ (обозначение: $F, x \vDash A$ ), если $A$ истинна в $x$ в любой модели Крипке на $F$. Множество формул Г общезначимо в $F$ (обозначение: $F \vDash \Gamma)$, если общезначимы все формулы из $Г$. Множество всех общезначимых в $F$ формул образует $n$-модальную логику $\mathbf{L}(F)$ (логику шкаль $F$ ). Логика класса $n$-шкал $\mathscr{C}$, или логика, задаваемая классом $\mathscr{C}$ (обозначение: $\mathbf{L}(\mathscr{C})$ ), определяется как пересечение всех логик шкал, входящих в $\mathscr{C}$.

Непосредственно из определений получаем следующее утверждение.

Лемма 2.2. $\mathbf{L}\left(\bigcup_{i \in I} \mathscr{C}_{i}\right)=\bigcap_{i \in I} \mathbf{L}\left(\mathscr{C}_{i}\right)$.

Обобщенная п-шкала Крипке - это $\left(W, R_{1}, \ldots, R_{n}, \mathscr{W}\right)$, где $\left(W, R_{1}, \ldots, R_{n}\right)$ шкала Крипке, $\mathscr{W} \subseteq \mathscr{P}(W)$ - множество ее подмножеств, замкнутое относительно булевых операций ("поле") и взятия прообразов $\left(R_{i}^{-1}\right)$. Оценка в обобщенной шкале $\Phi=\left(W, R_{1}, \ldots, R_{n}, \mathscr{W}\right)$ - это отображение $\theta: \mathrm{PV} \longrightarrow \mathscr{W}$. Формула $A$ общезначима в $\Phi$ (обозначение: $\Phi \vDash A$ ), если она истинна во всех моделях Крипке $\left(W, R_{1}, \ldots, R_{n}, \theta\right)$, где $\theta$ - оценка в $\Phi$. Таким образом, общезначимость в $F$ равносильна общезначимости в обобщенной шкале $(F, \mathscr{P}(W))$.

Модальная логика полна (по Крипке), если она представима в виде $\mathbf{L}(\mathscr{C})$ для некоторого класса (обычных) шкал Крипке $\mathscr{C}$. Логика какого-либо класса конечных (соответственно не более чем счетных) шкал называется финитно аппроксимируемой (соответственно счетно аппроксимируемой).

Хорошо известно, что всякая конечно аксиоматизируемая финитно аппроксимируемая модальная логика разрешима (теорема Харропа).

ЛЕмма 2.3. Если модальные логики $\boldsymbol{\Lambda}_{i}(i \in I)$ финитно аппроксимируемы, то $\bigcap_{i \in I} \boldsymbol{\Lambda}_{i}$ бинитно аппроксимируема. 
ДокаЗАТЕЛЬСтво. Пусть $\boldsymbol{\Lambda}_{i}=\mathbf{L}\left(\mathscr{C}_{i}\right)$ и $\mathscr{C}_{i}$ состоит из конечных шкал. По лемме $2.2, \bigcap_{i \in I} \boldsymbol{\Lambda}_{i}=\mathbf{L}\left(\bigcup_{i \in I} \mathscr{C}_{i}\right)$. При этом $\bigcup_{i \in I} \mathscr{C}_{i}-$ класс конечных шкал. Лемма доказана.

Обратно, для каждой $n$-модальной логики $\boldsymbol{\Lambda}$ можно рассмотреть класс $\mathbf{V}(\boldsymbol{\Lambda})$ всех $n$-шкал, на которых она общезначима; он называется многообразием шкал логики $\boldsymbol{\Lambda}$, а его элементы - $\boldsymbol{\Lambda}$-шкалами. Если $\boldsymbol{\Lambda}$ полна, то $\boldsymbol{\Lambda}=\mathbf{L}(\mathbf{V}(\boldsymbol{\Lambda}))$.

Для произвольного множества $n$-модальных формул $\Gamma$ также определяется многообразие шкал

$$
\mathbf{V}(\Gamma):=\{F \mid F-n \text {-шкала, } F \vDash \Gamma\} ;
$$

при этом $\mathbf{V}(\Gamma)=\mathbf{V}\left(\mathbf{K}_{n}+\Gamma\right)$ (теорема корректности). Обозначение $\mathbf{V}(\{A\})$ сокращается до $\mathbf{V}(A)$.

$n$-модальная формула $A$ называется элементарной, если класс $\mathbf{V}(A)$ элементарен, т. е. является классом $\operatorname{Mod}(\alpha)$ всех моделей некоторой классической формулы 1-го порядка $\alpha$ в сигнатуре $\mathscr{L} 1_{n}$ с $n$ двуместными предикатами и равенством. В этом случае говорят, что $\alpha$ coответствует A. Аналогично, модальная логика называется элементарной, если ее многообразие шкал элементарно. Модальная логика $\boldsymbol{\Lambda}$ называется $\Delta$-элементарной, если ее многообразие $\mathbf{V}(\boldsymbol{\Lambda}) \Delta$-элементарно, т. е. является классом всех моделей некоторой теории 1-го порядка соответствующей сигнатуры (или, эквивалентно, если $\mathbf{V}(\boldsymbol{\Lambda})$ есть пересечение элементарных классов).

Из теоремы Лёвенгейма-Сколема легко получается следующий факт [5; предложение 5.4].

Лемма 2.4. Всякая полная $\Delta$-элементарная модальная логика счетно аппроксимируема.

Напомним определение канонических шкал и моделей. Для $n$-модальной логики $\boldsymbol{\Lambda}$ каноническая шкала имеет вид $F_{\boldsymbol{\Lambda}}:=\left(W_{\boldsymbol{\Lambda}}, R_{1}, \ldots, R_{n}\right)$, где $W_{\boldsymbol{\Lambda}}-$ множество всех $\boldsymbol{\Lambda}$-полных теорий (т. е. максимальных непротиворечивых в $\boldsymbol{\Lambda}$ множеств формул), с отношениями

$$
x R_{i} y \Longleftrightarrow \forall A\left(\square_{i} A \in x \Rightarrow A \in y\right) .
$$

Каноническая модель Крипке определяется как $M_{\boldsymbol{\Lambda}}:=\left(F_{\boldsymbol{\Lambda}}, \theta_{\boldsymbol{\Lambda}}\right)$, где $\theta_{\boldsymbol{\Lambda}}\left(p_{k}\right):=$ $\left\{x \in W_{\boldsymbol{\Lambda}} \mid p_{k} \in x\right\}$. Обобщенная каноническая шкала - это $\Phi_{\boldsymbol{\Lambda}}:=\left(F_{\boldsymbol{\Lambda}}, \mathscr{W}_{\boldsymbol{\Lambda}}\right)$, где $\mathscr{W}_{\boldsymbol{\Lambda}}$ состоит из всех определимых подмножеств канонической модели:

$$
\mathscr{W}_{\Lambda}:=\left\{\theta_{\Lambda}(A) \mid A \in \mathscr{L}_{n}\right\},
$$

причем $\theta_{\boldsymbol{\Lambda}}(A):=\left\{x \mid M_{\boldsymbol{\Lambda}}, x \vDash A\right\}$.

Хорошо известна следующая теорема.

ТЕОРема 2.5 (теорема о канонической модели).

(i) $M_{\Lambda}, x \vDash A \Leftrightarrow A \in x$.

(ii) $M_{\boldsymbol{\Lambda}} \vDash A \Leftrightarrow \Phi_{\boldsymbol{\Lambda}} \vDash A \Leftrightarrow A \in \boldsymbol{\Lambda}$.

Для ограниченных логик также определяются канонические шкалы и моде-

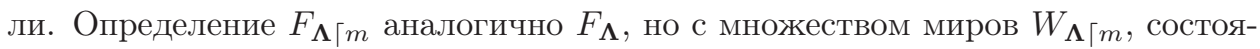
щим из всех $\boldsymbol{\Lambda}\lceil m$-полных теорий. 
Затем полагаем $M_{\boldsymbol{\Lambda}\lceil m}:=\left(F_{\boldsymbol{\Lambda}\lceil m}, \theta_{\boldsymbol{\Lambda}\lceil m}\right)$, где

$$
\theta_{\boldsymbol{\Lambda}\lceil m}\left(p_{k}\right):= \begin{cases}\left\{x \in W_{\boldsymbol{\Lambda}\lceil m} \mid p_{k} \in x\right\} & \text { при } k \leqslant m, \\ \varnothing & \text { при } k>m,\end{cases}
$$

и $\Phi_{\boldsymbol{\Lambda}\lceil m}:=\left(F_{\boldsymbol{\Lambda}\lceil m}, \mathscr{W}_{\boldsymbol{\Lambda}\lceil m}\right)$, где $\mathscr{W}_{\boldsymbol{\Lambda}\lceil m}:=\left\{\theta_{\boldsymbol{\Lambda}\lceil m}(A) \mid A \in \mathscr{L}_{n}\lceil m\}\right.$.

ТЕорема 2.6 (теорема об ограниченной канонической модели). Для $A \in$ $\mathscr{L}_{n}\lceil m$ имеем

(i) $M_{\Lambda\lceil m}, x \vDash A \Longleftrightarrow A \in x$;

(ii) $M_{\Lambda\lceil m} \vDash A \Longleftrightarrow \Phi_{\Lambda\lceil m} \vDash A \Longleftrightarrow A \in \Lambda\lceil m$;

(iii) $\Phi_{\Lambda\lceil m} \vDash \Lambda$.

Остановимся кратко на доказательстве утверждения (iii). Пусть $\psi$ - оценка в $\Phi_{\boldsymbol{\Lambda}\lceil m}$, и пусть

$$
\psi\left(p_{i}\right)=\theta_{\boldsymbol{\Lambda}\lceil m}\left(B_{i}\right), \quad B_{i} \in \mathscr{L}_{n}\lceil m .
$$

Тогда индукцией по построению $A\left(p_{1}, \ldots, p_{k}\right)$ (где $k$ - произвольное) получается, что

$$
\psi\left(A\left(p_{1}, \ldots, p_{k}\right)\right)=\theta_{\boldsymbol{\Lambda}\lceil m}\left(A\left(B_{1}, \ldots, B_{k}\right)\right) .
$$

Поэтому, если $A \in \boldsymbol{\Lambda}$, то $A\left(B_{1}, \ldots, B_{k}\right) \in \boldsymbol{\Lambda}\lceil m$ и, следовательно,

$$
\psi\left(A\left(p_{1}, \ldots, p_{k}\right)\right)=\theta_{\boldsymbol{\Lambda}\lceil m}\left(A\left(B_{1}, \ldots, B_{k}\right)\right)=W_{\boldsymbol{\Lambda}\lceil m},
$$

ввиду утверждения (ii).

Напомним, что модальная логика $\boldsymbol{\Lambda}$ называется канонической, если $F_{\boldsymbol{\Lambda}} \vDash \boldsymbol{\Lambda}$. Ввиду теоремы о канонической модели, это равносильно $\boldsymbol{\Lambda}=\mathbf{L}\left(F_{\boldsymbol{\Lambda}}\right)$, и, следовательно, всякая каноническая логика полна.

Для обобщенной шкалы $\left(W, R_{1}, \ldots, R_{n}, \mathscr{W}\right)$ обозначим

$$
\mathscr{W}_{x}:=\{V \in \mathscr{W} \mid x \in V\} .
$$

ОПРеДЕЛЕНиЕ 2.7. Обобщенная шкала $\left(W, R_{1}, \ldots, R_{n}, \mathscr{W}\right)$ называется дескриптивной, если выполняются следующие условия:

- $($ различимость $) \forall x, y\left(x \neq y \Rightarrow \mathscr{W}_{x} \neq \mathscr{W}_{y}\right)$,

- (тугость) $\forall i, x, y\left(x \in \bigcap\left\{R_{i}^{-1}(V) \mid V \in \mathscr{W}_{y}\right\} \Rightarrow x R_{i} y\right)$,

- (компактность) если $\mathscr{V} \subseteq \mathscr{W}$ центрировано (т. е. пересечение всякого конечного подмножества $\mathscr{V}$ непусто), то $\bigcap \mathscr{V} \neq \varnothing$.

ОПРЕДЕЛЕНИЕ 2.8. Модальная логика $\boldsymbol{\Lambda}$ называется d-yпорной, если для любой дескриптивной обобщенной шкалы $\Phi=(F, \mathscr{W})$ из $\Phi \vDash \boldsymbol{\Lambda}$ следует $F \vDash \boldsymbol{\Lambda}$.

Поскольку $\Phi_{\boldsymbol{\Lambda}} \vDash \boldsymbol{\Lambda}$, из d-упорства логики следует ее каноничность.

Теорема Салквиста (в ее современной формулировке) дает достаточное условие d-упорства. Формуль Салквиста - это модальные формулы специального вида; в частности, любая формула вида $\square_{\alpha}\left(\diamond_{\beta} \square_{\gamma} p \supset B\right)$, где $B$ - позитивная, является формулой Салквиста [17].

Теорема 2.9 (теорема Салквиста в форме Самбина-Ваккаро). Всякая модальная логика, аксиоматизируемая формулами Салквиста, $\Delta$-элементарна и d-упорна. 
Следующая теорема (см. [18], [17]) дает более общее достаточное условие d-упорства.

Теорема 2.10 (теорема Файна-Ван Бентема). Всякая полная $\Delta$-элементарная модальная логика d-упорна.

Еще одно простое замечание будет полезно в дальнейшем.

Лемма 2.11. 1) Если $L_{1}, L_{2}$ суть d-упорные $n$-модальные логики, то и логика $L_{1}+L_{2}$ является d-упорной.

2) Если $L_{1}, L_{2}$ суть d-упорные модальные логики, то и логика $L_{1} * L_{2}$ является d-упорной.

ДоказАтельство. Утверждения достаточно очевидны. Утверждение 1) следует из того, что общезначимость $L_{1}+L_{2}$ в обобщенной шкале Крипке равносильна общезначимости обеих логик $L_{1}$ и $L_{2}$. Для доказательства 2) достаточно заметить, что (для $n$-модальной $L_{1}$ и $m$-модальной $L_{2}$ )

$$
\begin{aligned}
& \left(W, R_{1}, \ldots, R_{n}, R_{n+1}, \ldots, R_{n+m}, \mathscr{W}\right) \vDash L_{1} * L_{2} \\
& \quad \Longleftrightarrow\left(W, R_{1}, \ldots, R_{n}, \mathscr{W}\right) \vDash L_{1} \&\left(W, R_{n+1}, \ldots, R_{n+m}, \mathscr{W}\right) \vDash L_{2} .
\end{aligned}
$$

Лемма доказана.

Напомним, что подикала шкалы $F=\left(W, R_{1}, \ldots, R_{n}\right)$ имеет вид $F\lceil V:=$ $\left(V, R_{1} \uparrow V, \ldots, R_{n} \uparrow V\right)$ для некоторого $V \subseteq W$. Если $M=(F, \theta)$ - модель Крипке, то определяем ее подмодель $M \uparrow V:=\left(F \nmid V, \theta\lceil V)\right.$, где $\left(\theta\lceil V)\left(p_{i}\right):=\theta\left(p_{i}\right) \cap V\right.$.

Если $R_{i}(V) \subseteq V$ для всех $i$, то $V$ называется коническим подмножеством, а $F \nmid V$ - конической подшкалой. Конус в $F$ с корнем $u$ - это подшкала вида $F \nmid R^{*}(u)$, где $R^{*}$ - рефлексивное транзитивное замыкание $R_{1} \cup \cdots \cup R_{n}$; она обозначается также $F \uparrow u$.

Очевидно, что $R^{*}(u)$ - наименьшее коническое подмножество, содержащее $u$.

Также очевидно, что $u R^{*} y$, если и только если в $F$ имеется nymъ из $x$ в $y$, т. е. последовательность точек $x=x_{0}, \ldots, x_{k}=y$ такая, что для всех $j<k$ $x_{j}\left(R_{1} \cup \cdots \cup R_{n}\right) x_{j+1}$ или же $k=0$ (и тогда $x=x_{0}=y$ ). Отсюда получается следующее утверждение.

Лемма 2.12. В шкале $\left(W, R_{1}, \ldots, R_{n}\right)$

$$
R^{*}(u)=\{u\} \cup \bigcup\left\{R^{*}(x) \mid x \in R_{1}(u) \cup \cdots \cup R_{n}(u)\right\} .
$$

Если $F=F \uparrow u$ для некоторого $u$, то сама $F$ называется конусом ( Аналогичные термины и обозначения используются для моделей Крипке.

ЛЕмма 2.13 (лемма о конусах). (i) Если $F\lceil V$ - коническал подикала $F$, то $\mathbf{L}(F) \subseteq \mathbf{L}(F\lceil V)$; более того, если $x \in V$ и $F, x \vDash A$, то $F\lceil V, x \vDash A$.

(ii) $\mathbf{L}(F)=\bigcap_{u \in W} \mathbf{L}(F \uparrow u)$.

(iii) Если $M \uparrow V$ - коническая подмодель $M$, то для любой модалъной формульь $A$

$$
M, x \vDash A \Longleftrightarrow M\lceil V, x \vDash A .
$$

Таким образом, всякая полная логика задается некоторым классом конусов. Напомним также понятие бисимуляции. 
ОПРЕДЕЛЕНИЕ 2.14. (Частичная) бисимуляиия между моделями Крипке $M=\left(W, R_{1}, \ldots, R_{n}, \theta\right)$ и $M^{\prime}=\left(W^{\prime}, R_{1}^{\prime}, \ldots, R_{n}^{\prime}, \theta^{\prime}\right)$ - это отношение $E \subseteq W \times W^{\prime}$ со следующими свойствами (для всех $i \in\{1, \ldots, n\}, q \in \mathrm{PV}$ ):

$(\rightarrow) E^{-1} \circ R_{i} \subseteq R_{i}^{\prime} \circ E_{i}^{-1}$

$(\leftarrow) E \circ R_{i}^{\prime} \subseteq R_{i} \circ E$;

(pv) если $x E x^{\prime}$, то $M, x \vDash q \Longleftrightarrow M^{\prime}, x^{\prime} \vDash q$.

Запись $E: M, x \asymp M^{\prime}, x^{\prime}$ означает, что $E$ - бисимуляция между $M$ и $M^{\prime}$ такая, что $x E x^{\prime}$.

Условия $(\rightarrow)$ и $(\leftarrow)$ проиллюстрированы на рис. 1.

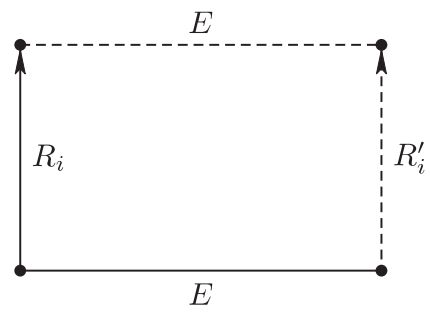

$(\rightarrow)$

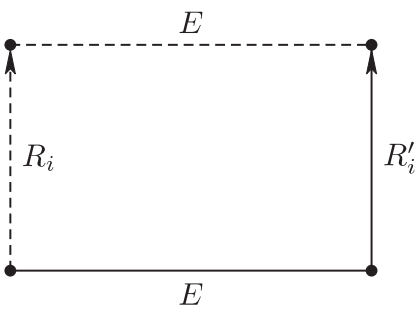

$(\leftarrow)$

Рис. 1

Если бисимуляция $E$ является сюръективной функцией $W \longrightarrow W^{\prime}$, то она называется р-морбизмом $M$ на $N$ (и соответствующих шкал). В этом случае условие $(\rightarrow)$ превращается в монотонность:

$$
x R_{i} y \Longrightarrow E(x) R_{i}^{\prime} E(y),
$$

а условие $(\leftarrow)$ - в свойство поднятия:

$$
E(x) R_{i}^{\prime} z \Longrightarrow \exists y\left(x R_{i} y \& E(y)=z\right) .
$$

Запись $E: M \rightarrow M^{\prime}$ означает, что $E-$ р-морфизм модели $M$ на $M^{\prime}$; аналогичное обозначение используется для р-морфизмов шкал.

Частным случаем р-морфизма является изоморфизм; тогда $E$ - биекция, а конъюнкция условий $(\leftarrow),(\rightarrow)$ принимает вид

$$
\forall x \forall y\left(x R_{i} y \Leftrightarrow E(x) R_{i}^{\prime} E(y)\right) .
$$

Заметим еще, что если $R_{i}=f, R_{i}^{\prime}=f^{\prime}-$ функции, то конъюнкцию $(\leftarrow) \&(\rightarrow)$ можно записать так:

$$
\forall x \forall x^{\prime}\left(x E x^{\prime} \Rightarrow f(x) E f^{\prime}\left(x^{\prime}\right)\right) .
$$

ЛЕмма 2.15 (лемма о бисимуляции). Если $E: M, x \asymp N, y$ для $n$-модальных моделей Крипке $M, N$, mо $M, x \vDash A \Leftrightarrow N, y \vDash A$ для любой $n$-модальной формуль $A$.

Этот факт хорошо известен (см., например, [19; теорема 2.20]), так же как и следующая лемма. 
ЛЕмма 2.16 (лемма о р-морфизме). Если $F, G$ - $n$-шкаль $u f: F \rightarrow G, m o$

(i) $\mathbf{L}(F) \subseteq \mathbf{L}(G)$; более того, если $F, x \vDash A$, то $G, f(x) \vDash A$;

(ii) $F \vDash A \Leftrightarrow G \vDash A$ для любой замкнутой $n$-модальной формуль $A$.

ОПРЕДЕЛЕНиЕ 2.17. Модальная логика называется локально табличной, если все ее ограниченные канонические модели конечны.

По теореме об ограниченной канонической модели, это равносильно тому, что для любого $m$ в данной логике имеется лишь конечное число неэквивалентных формул с переменными $p_{1}, \ldots, p_{m}$ : действительно, такие формулы $A, B$ эквивалентны в $\boldsymbol{\Lambda}$, если и только если $\theta_{\boldsymbol{\Lambda}\lceil m}(A)=\theta_{\boldsymbol{\Lambda}\lceil m}(B)$.

ЛЕмма 2.18. Всякая локально табличная модальная логика финитно аппроксимируема.

ДоказАтЕльство. Если $\Phi_{\boldsymbol{\Lambda}\lceil m}$ конечна, то, в силу ее различимости, $\mathscr{W}_{\boldsymbol{\Lambda}}\lceil m$ состоит из всех подмножеств. Поэтому в $\Phi_{\boldsymbol{\Lambda}}\left\lceil m\right.$ и $F_{\boldsymbol{\Lambda}}\lceil m$ общезначимы одни и те же формулы. Тогда по теореме 2.6 (iii) $F_{\boldsymbol{\Lambda}\lceil m} \vDash \boldsymbol{\Lambda}$. Это означает, что

$$
\boldsymbol{\Lambda} \subseteq \mathbf{L}\left(\left\{F_{\boldsymbol{\Lambda}\lceil m} \mid m \geqslant 0\right\}\right) .
$$

Но обратное включение верно для любой логики, в силу теоремы 2.6 (ii). Поэтому

$$
\boldsymbol{\Lambda}=\mathbf{L}\left(\left\{F_{\boldsymbol{\Lambda}\lceil m} \mid m \geqslant 0\right\}\right),
$$

и, значит, $\boldsymbol{\Lambda}$ - логика класса конечных шкал. Лемма доказана.

ОПРедЕЛЕНИЕ 2.19. Произведением шкал Крипке $F=\left(W, R_{1}, \ldots, R_{n}\right)$ и $G=\left(V, S_{1}, \ldots, S_{m}\right)$ называется шкала

$$
F \times G=\left(W \times V, R_{11}, \ldots, R_{n 1}, S_{12}, \ldots, S_{m 2}\right),
$$

где

$$
\begin{aligned}
& (x, y) R_{i 1}\left(x^{\prime}, y^{\prime}\right) \Longleftrightarrow x R_{i} x^{\prime} \& y=y^{\prime}, \\
& (x, y) S_{j 2}\left(x^{\prime}, y^{\prime}\right) \Longleftrightarrow x=x^{\prime} \& y S_{j} y^{\prime} .
\end{aligned}
$$

ОПРЕДЕЛЕНИЕ 2.20. Пусть $\mathscr{C}_{1}, \mathscr{C}_{2}$ - классы соответственно $n$-шкал и $m$-шкал Крипке. Их произведением называется класс $(n+m)$-шкал

$$
\mathscr{C}_{1} \times \mathscr{C}_{2}:=\left\{F_{1} \times F_{2} \mid F_{1} \in \mathscr{C}_{1}, F_{2} \in \mathscr{C}_{2}\right\} .
$$

ОПРЕДЕЛЕНИЕ 2.21. Пусть $\boldsymbol{\Lambda}_{1}, \boldsymbol{\Lambda}_{2}-n$-модальная и $m$-модальная логика соответственно, причем $\mathbf{V}\left(\boldsymbol{\Lambda}_{1}\right), \mathbf{V}\left(\boldsymbol{\Lambda}_{2}\right) \neq \varnothing$. Их произведение - это $(n+m)$-модальная логика

$$
\boldsymbol{\Lambda}_{1} \times \boldsymbol{\Lambda}_{2}:=\mathbf{L}\left(\mathbf{V}\left(\boldsymbol{\Lambda}_{1}\right) \times \mathbf{V}\left(\boldsymbol{\Lambda}_{2}\right)\right) .
$$

ОПРЕДЕЛЕНИЕ 2.22. Коммутативное соединение ("коммутант") n-модальной логики $\boldsymbol{\Lambda}_{1}$ и $m$-модальной логики $\boldsymbol{\Lambda}_{2}$ определяется следующим образом:

$$
\begin{aligned}
{\left[\boldsymbol{\Lambda}_{1}, \boldsymbol{\Lambda}_{2}\right]:=\boldsymbol{\Lambda}_{1} * \boldsymbol{\Lambda}_{2} } & +\left\{\mathrm{Com}_{i j} \mid 1 \leqslant i \leqslant n, 1 \leqslant j \leqslant m\right\} \\
& +\left\{\mathrm{CR}_{i j} \mid 1 \leqslant i \leqslant n, 1 \leqslant j \leqslant m\right\},
\end{aligned}
$$


где

$$
\begin{aligned}
& \operatorname{Com}_{i j}:=\left(\square_{i} \square_{n+j} p \equiv \square_{n+j} \square_{i} p\right) \text { (аксиомы коммутирования), } \\
& \mathrm{CR}_{i j}:=\left(\diamond_{i} \square_{n+j} p \supset \square_{n+j} \diamond_{i} p\right) \quad \text { (аксиомы конвергенции, или Чёрча-Россера). }
\end{aligned}
$$

Логики $\boldsymbol{\Lambda}_{1}, \boldsymbol{\Lambda}_{2}$ называются хорошо перемножсаемыми (англ. - product-matching), если $\boldsymbol{\Lambda}_{1} \times \boldsymbol{\Lambda}_{2}=\left[\boldsymbol{\Lambda}_{1}, \boldsymbol{\Lambda}_{2}\right]$.

Как легко видеть, аксиомы коммутирования и конвергенции элементарны; их многообразия задаются следующими условиями:

$$
\begin{aligned}
\left(W, R_{1}, \ldots, R_{n}\right) \vDash \mathrm{Com}_{i j} & \Longleftrightarrow R_{i} \circ R_{n+j}=R_{n+j} \circ R_{i}, \\
\left(W, R_{1}, \ldots, R_{n}\right) \vDash \mathrm{CR}_{i j} & \Longleftrightarrow R_{i}^{-1} \circ R_{n+j} \subseteq R_{n+j} \circ R_{i}^{-1} .
\end{aligned}
$$

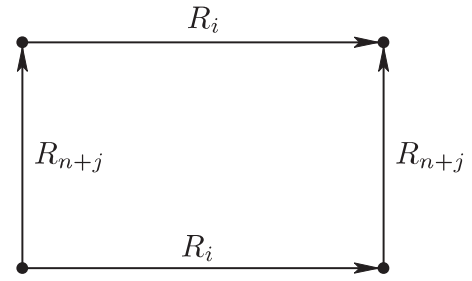

$\left(\mathrm{Com}_{i j}\right)$

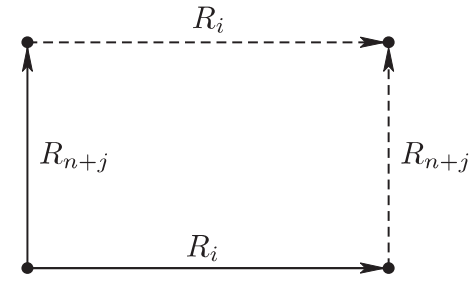

$\left(\mathrm{CR}_{i j}\right)$

Рис. 2

Напомним определение логики "завтра" (введенной К. Сегербергом [20]):

$$
\mathbf{S L}:=\mathbf{K}+\square p \equiv \diamond p .
$$

Как известно, $\mathbf{S L - ш к а л ы ~ и м е ю т ~ в и д ~}(W, R)$, где $R$ - график функции $W \longrightarrow W$ (в дальнейшем такая функция отождествляется с ее графиком). Также известна следующая теорема полноты [20].

ПрЕДЛОЖЕНИЕ 2.23. $\mathbf{S L}=\mathbf{L}(\omega, S)$, где $S$ - бункция следования $(S(x)=$ $x+1)$.

$\mathbf{S L}_{n}$ обозначает $n$-кратное соединение $\mathbf{S L} * \cdots * \mathbf{S L}$.

Теперь напомним определение модальных логик хорновского типа [4].

ОПРЕДЕЛЕНИЕ 2.24. (Строго) хорновское предложение - это $\mathscr{L} 1_{n}$-предложение вида $\bar{\forall}\left(\varphi \supset P_{i}(x, y)\right)$, где $\bar{\forall}$ - универсальное замыкание, $\varphi$ - бескванторная позитивная формула (т. е. построенная из атомарных формул с помощью конъюнкции и дизъюнкции), $P_{i}$ - предикатный символ, отличный от равенства, $x, y$ - различные переменные. Множество хорновских предложений называется хорновской теорией.

ОПРЕДЕЛЕНИЕ 2.25. Пусть $F_{1}=\left(W_{1}, S_{1}, \ldots, S_{n}\right), F_{2}=\left(W_{2}, S_{1}^{\prime}, \ldots, S_{n}^{\prime}\right)-$ $n$-модальные шкалы. Мы называем $F_{2}$ слабым расширением $F_{1}$ (обозначение: $\left.F_{1} \subseteq F_{2}\right)$, если $W_{1}=W_{2}$ и $S_{i} \subseteq S_{i}^{\prime}$ для всех $i$.

Следующая лемма позволяет строить "хорновское замыкание" - наименьшее слабое расширение шкалы, удовлетворяющее данной хорновской теории; доказательство леммы см., например, в [5]. 
Лемма 2.26. Пусть $F=\left(W, \rho_{1}, \ldots, \rho_{n}\right)-$ шкала Крипке $u \Gamma=\left\{\psi_{k} \mid k \in I\right\}-$ хорновская теория,

$$
\psi_{k}=\bar{\forall}\left(\varphi_{k} \supset R_{i_{k}}(x, y)\right) .
$$

Тогда существует шкала $F_{\Gamma}^{+}$такая, что

1) $F \subseteq F_{\Gamma}^{+}$;

2) $F_{\Gamma}^{+} \vDash \Gamma$;

3) если $G \vDash \Gamma$ и $f: F \longrightarrow G$ - монотонное отображение, то оно же $u$ монотонное отображение $F_{\Gamma}^{+} \longrightarrow G$.

ОПредЕлЕниЕ 2.27. Модальная формула называется хорновской, если она соответствует хорновскому предложению. Модальная логика хорновского типа (англ. - Horn axiomatizable) получается из минимальной добавлением аксиом, которые являются замкнутыми или хорновскими формулами.

Типичные примеры хорновских формул - псевдотранзитивные формулы из работы [5].

ОПРЕДЕЛЕНИЕ 2.28. Модальная формула называется псевдотранзитивной, если она имеет вид $\diamond_{\alpha} \square_{k} p \supset \square_{\beta} p$, где $p \in \mathrm{PV}, \alpha, \beta$ - последовательности индексов. РТC-логика (от "pseudo-transitive or closed") - это модальная логика, задаваемая множеством аксиом, которые псевдотранзитивны или замкнуты.

ПредложениЕ 2.29. 1) Псевдотранзитивная формула $A=\diamond_{\alpha} \square_{k} p \supset \square_{\beta} p$ соответствует хорновскому предложению

$$
\varphi=\forall x \forall v\left(\exists u\left(u R_{\alpha} x \wedge u R_{\beta} v\right) \supset R_{k}(x, v)\right),
$$

¿де

$$
R_{i_{1} \ldots i_{r}}:=R_{i_{1}} \circ \cdots \circ R_{i_{r}} .
$$

2) Всякая РТС-логика - каноническая.

Здесь $u R_{\alpha} x, u R_{\beta} v$ следует понимать как сокращенные записи некоторых экзистенциальных формул; таким образом, в предваренной форме $\varphi$ становится хорновским предложением. Если $\alpha$ пуста, то $R_{\alpha}$ понимается как равенство.

Среди РТС-логик оказываются хорошо известные, например, $\mathbf{D}=\mathbf{K}+\diamond \top$, $\mathbf{T}=\mathbf{K}+\square p \supset p, \mathbf{K} 4, \mathbf{S} 4, \mathbf{K} . \mathbf{t}, \mathbf{S} 5$ и др.

Однако не все хорновские модальные формулы псевдотранзитивны. Примерами могут служить

$$
\square\left(\square p \supset \square^{2} p\right),
$$

которой соответствует

$$
\bar{\forall}(R(x, y) \wedge R(y, z) \wedge R(z, t) \supset R(y, t)),
$$

и

$$
\diamond^{2}(p \wedge \diamond \top) \supset \diamond p
$$

которой соответствует

$$
\bar{\forall}(R(x, y) \wedge R(y, z) \wedge R(z, t) \supset R(x, z)) .
$$

С другой стороны, недавно было установлено, что всякое хорновское предложение соответствует некоторой модальной формуле [21]. 
Теорема 2.30. Любъе две полные модалъные логики хорновского типа хорошо перемножаемы.

Доказательство см. в [4]; по существу оно повторяется в настоящей работе в процессе доказательства теоремы 4.2.

\section{3. Квадраты Сегерберга}

ОПРедЕЛЕНиЕ 3.1. Квадратом Сегерберга шкалы Крипке $F=\left(W, R_{1}, \ldots, R_{n}\right)$ называется $(2 n+3)$-шкала

$$
F_{\mathrm{Sg}}^{2}:=\left(F^{2}, \sigma_{0}, \sigma_{1}, \sigma_{2}\right),
$$

которая получается из $F^{2}=F \times F$ добавлением функций

$$
\begin{aligned}
& \sigma_{0}:(x, y) \mapsto(y, x) \text { (симметрия относительно диагонали), } \\
& \sigma_{1}:(x, y) \mapsto(y, y) \text { (горизонтальная проекция на диагональ), } \\
& \sigma_{2}:(x, y) \mapsto(x, x) \text { (вертикальная проекция на диагональ). }
\end{aligned}
$$

ОПРЕДЕЛЕНИЕ 3.2. Квадратом Сегерберга $n$-модальной логики $\boldsymbol{\Lambda}$ называется логика

$$
\boldsymbol{\Lambda}_{\mathrm{Sg}}^{2}:=\mathbf{L}\left(\left\{F_{\mathrm{Sg}}^{2} \mid F \vDash \boldsymbol{\Lambda}\right\}\right) .
$$

Таким образом, $\boldsymbol{\Lambda}_{\mathrm{Sg}}^{2}$ есть $(2 n+3)$-модальная логика. Ее связки обозначаются $\boxminus_{1}, \ldots, \boxminus_{n}, \square_{1}, \ldots, \square_{n}, \bigcirc, \oplus, \ominus$. Если $F=\left(W, R_{1}, \ldots, R_{n}\right)$ - шкала Крипке, то соответствующие модальные формулы интерпретируются в модели Крипке на квадрате Сегерберга $F_{\mathrm{Sg}}^{2}$ следующим образом:

$$
\begin{aligned}
& (x, y) \vDash \boxminus_{i} A \Longleftrightarrow \forall z\left(x R_{i} z \Rightarrow(z, y) \vDash A\right), \\
& (x, y) \vDash \square_{i} A \Longleftrightarrow \forall z\left(y R_{i} z \Rightarrow(x, z) \vDash A\right), \\
& (x, y) \vDash \bigcirc A \Longleftrightarrow \sigma_{0}(x, y) \vDash A, \\
& (x, y) \vDash \bigcirc A \Longleftrightarrow \sigma_{1}(x, y) \vDash A, \\
& (x, y) \vDash \ominus A \Longleftrightarrow \sigma_{2}(x, y) \vDash A .
\end{aligned}
$$

Отметим, что в работе Сегерберга [13] рассматривается только случай, когда $n=1$, а отношение $R_{1}$ в исходной шкале $F$ - универсальное. Связка $\bigcirc$ там обозначается $\bigotimes$, а индексы 1 у $\boxminus$ и $\square$ опускаются.

Далее в этой работе мы используем обозначение $\bigcirc_{1}$ для $\bigcirc$ и $\bigcirc_{2}-$ для $\ominus$.

Выпишем теперь модальные формулы, которые понадобятся для аксиоматизации квадратов Сегерберга. Поскольку $\sigma_{1}, \sigma_{2}, \sigma_{0}-$ функции, для связок $\bigcirc_{1}, \bigcirc_{2}, \bigcirc$ выполняются аксиомы SL, т. е. квадрат Сегерберга является $\mathbf{K}_{2 n} * \mathbf{S L}_{3}$-шкалой. Имеются также следующие дополнительные аксиомы: 


$$
\begin{aligned}
& \text { (Sg1) } \bigcirc^{2} p \equiv p, \\
& (\text { Sg2 }) \bigcirc_{1}^{2} p \equiv \bigcirc_{1} p, \\
& \text { (Sg3) } \bigcirc_{2} p \equiv \bigcirc_{1} p, \\
& \text { (Sg4) } \bigcirc_{1} \bigcirc p \equiv \bigcirc_{1} p, \\
& \text { (Sg5) } \square_{i} p \equiv \boxminus_{i} \bigcirc p, \\
& \text { (Sg6) } \bigcirc_{1} \boxminus_{i}\left(\square_{i} p \supset \bigcirc_{2} p\right), \\
& \text { (Sg7) } \bigcirc_{1} p \supset \boxminus_{i} \bigcirc_{1} p, \\
& \left(\text { Sg8) } \square_{i} \bigcirc_{1} p \equiv \bigcirc_{1} \varpi_{i} \bigcirc_{1} p .\right.
\end{aligned}
$$

Все эти аксиомы являются формулами Салквиста и, следовательно, элементарны. Приведем описание соответствующих классических формул.

Лемма 3.3. 1) Пусть $G=\left(V, X_{1}, \ldots, X_{n}, Y_{1}, \ldots, Y_{n}, f_{0}, f_{1}, f_{2}\right) \vDash \mathbf{K}_{2 n} * \mathbf{S L}_{3}$. Тогда

$$
\begin{aligned}
G \vDash(\operatorname{Sg} 1) \Longleftrightarrow & f_{0} f_{0}=1_{V}, \\
G \vDash(\operatorname{Sg} 2) \Longleftrightarrow & f_{1} f_{1}=f_{1}, \\
G \vDash(\operatorname{Sg} 3) \Longleftrightarrow & f_{1} f_{0}=f_{2}, \\
G \vDash(\operatorname{Sg} 4) \Longleftrightarrow & f_{0} f_{1}=f_{1}, \\
G \vDash(\operatorname{Sg} 5) \Longleftrightarrow & \forall a, b \in V\left(a X_{i} b \Leftrightarrow f_{0}(a) Y_{i} f_{0}(b)\right) \\
& (\text { при условии } G \vDash(\operatorname{Sg} 1)), \\
G \vDash(\operatorname{Sg} 6) \Longleftrightarrow & \forall a, b \in V\left(f_{1}(a) X_{i} b \Rightarrow b Y_{i} f_{2}(b)\right), \\
G \vDash(\operatorname{Sg} 7) \Longleftrightarrow & \forall a, b \in V\left(a X_{i} b \Rightarrow f_{1}(a)=f_{1}(b)\right), \\
G \vDash(\operatorname{Sg} 8) \Longleftrightarrow & \forall a \in V f_{1}\left[Y_{i}(a)\right]=f_{1}\left[Y_{i}\left(f_{1}(a)\right)\right] .
\end{aligned}
$$

2) Аксиомы (Sg1)-(Sg8) общезначимы в квадратах Сегерберга.

ПРИМЕЧАНИЕ. В отличие от композиции отношений, мы обозначаем композицию функций $f: U \longrightarrow V$ и $g: V \longrightarrow W$ через $g f$. Тождественная функция $V \longrightarrow V$ обозначается через $1_{V}$. Образ множества $Z$ при отображении $f$ обозначается через $f[Z]$.

Доказательство леммы стандартно, и мы его опускаем. Однако обсудим некоторые простые ее следствия.

Аксиома (Sg1) означает, что $f_{0}$ - инволюция на $V$; в квадратах Сегерберга это симметрия относительно диагонали. Неподвижные точки $f_{0}$ назовем слабо диагональными; в квадратах Сегерберга они составляют диагональ.

Аксиома (Sg2) означает, что $f_{1}$ - идемпотентное отображение $V$ в себя.

Аксиома $(\mathrm{Sg} 3)$ позволяет выразить $\bigcirc_{2}$ через $\bigcirc_{1}$ и $\bigcirc$ и, аналогично, $f_{2}$ чеpeз $f_{1}, f_{0}$.

Аксиома (Sg4) означает, что образ $f_{1}$ состоит из слабо диагональных точек, а из $(\mathrm{Sg} 3)$ следует, что он совпадает с образом $f_{2}$. Точки вида $f_{1}(a)$ назовем диагональными. В квадратах Сегерберга все слабо диагональные точки диагональны, но в общем случае это не обязательно.

Из общезначимости аксиом (Sg3) и (Sg4) получаем:

$$
f_{0} f_{1} f_{0}=f_{2},
$$

т. е. $f_{1}$ и $f_{2}$ сопряжены посредством инволюции $f_{0}$. 
В любом множестве вида $W^{2}$ диагональная симметрия, две проекции на диагональ и тождественное отображение образуют моноид порядка 4. Он порождается двумя образующими $\sigma_{0}, \sigma_{1}$ и соотношениями

$$
\sigma_{0}^{2}=1, \quad \sigma_{1}^{2}=\sigma_{1}, \quad \sigma_{0} \sigma_{1}=\sigma_{1},
$$

которые в точности соответствуют аксиомам (Sg1), (Sg2), (Sg3).

Из аксиом (Sg5) и (Sg1) получаем, что $f_{0}$ - изоморфизм между отношениями $X_{i}$ и $Y_{i}$. Это позволяет выразить вертикальные модальности через горизонтальные и связку $\bigcirc$; таким образом, квадрат Сегерберга по существу является не $(2 n+3)$-, а $(n+2)$-модальной логикой.

Аксиома (Sg5) также означает, что отношения $X_{i}$ и $Y_{i}$ сопряжены посредством $f_{0}$ в следующем смысле:

$$
f_{0} \circ X_{i} \circ f_{0}=Y_{i}
$$

(здесь о- композиция отношений). В случае, когда $X_{i}$ и $Y_{i}$ являются функциями, это - сопряженность в моноиде отображений $V \longrightarrow V$.

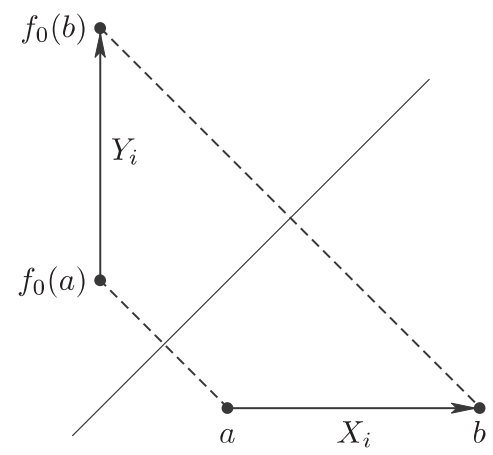

Рис. 3. Условие (Sg5)

Используя (Sg3), (Sg5), (Sg7), (Sg2), условие общезначимости для (Sg6) можно переписать в следующем виде:

$$
\forall a \in V\left(f_{1}(a) X_{i} a \Rightarrow f_{0}(a) X_{i} f_{1} f_{0}(a)\right) .
$$

В частности, в квадратах Сегерберга, если точка $(x, y)$ горизонтально достижима из диагональной (т. е. $y R_{i} x$ для некоторого $i$ ), то, наоборот, из симметричной точки $(y, x)$ горизонтально достижима диагональная.

Аксиома (Sg7) в случае квадратов Сегерберга означает, что точки, связанные горизонтальной достижимостью, находятся на одной горизонтали, т. е. их горизонтальные проекции на диагональ совпадают.

Аксиома (Sg8) для квадратов Сегерберга означает, что для любой точки $(x, y)$ горизонталь любой вертикально достижимой точки (т. е. точки $(x, z)$, где $\left.y R_{i} z\right)$ обязательно содержит точку, которая вертикально достижима из диагональной $(y, y)$, и наоборот, горизонталь любой точки, вертикально достижимой из $(y, y)$, содержит точку, вертикально достижимую из $(x, y)$. 


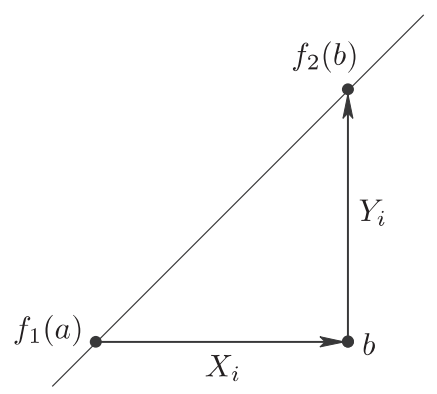

Рис. 4. Условие (Sg6)

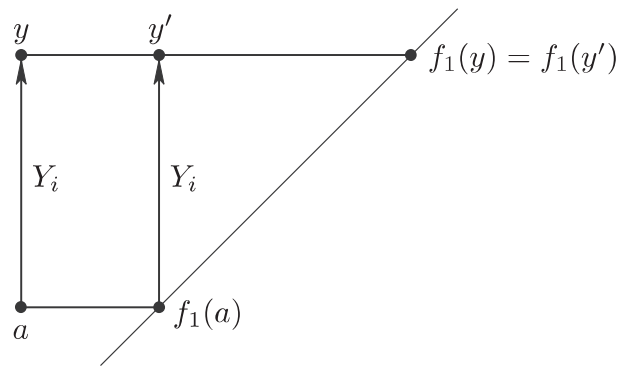

Рис. 5. Условие (Sg8)

В языке логики $\mathbf{K}_{2 n} * \mathbf{S L}_{3}$ с горизонтальными и вертикальными модальностями для каждой формулы $A$ имеется сопряженная формула $A^{\circ}$; она получается заменой вертикальных модальностей на горизонтальные и наоборот. Формальное определение - по индукции.

ОПреДЕЛЕниЕ 3.4. $A^{\circ}:=A$, если $A$ - атомарная формула,

$$
\begin{aligned}
& (A \supset B)^{\circ}:=A^{\circ} \supset B^{\circ}, \quad\left(\boxminus_{i} A\right)^{\circ}:=\square_{i} A^{\circ}, \quad\left(\square_{i} A\right)^{\circ}:=\boxminus_{i} A^{\circ}, \\
& \left(\bigcirc_{1} A\right)^{\circ}:=\bigcirc_{2} A^{\circ}, \quad\left(\bigcirc_{2} A\right)^{\circ}:=\bigcirc_{1} A^{\circ}, \quad(\bigcirc A)^{\circ}:=\bigcirc A^{\circ} \text {. }
\end{aligned}
$$

ЛЕмма 3.5 (принцип сопряженности). Пусть $L$ есть $(2 n+3)$-модальная логика со связками $\boxminus_{i}, \square_{i}, \bigcirc_{1}, \bigcirc_{2}, \bigcirc$, содержащая $\mathbf{K}_{2 n} * \mathbf{S L}_{3}+\{(\mathrm{Sg} 1),(\mathrm{Sg} 3)$, $(\mathrm{Sg} 4),(\mathrm{Sg} 5)\}$. Тогда

1) для любой формуль $A\left(p_{1}, \ldots, p_{n}\right)$

$$
L \vdash A^{\circ} \equiv \bigcirc A\left(\bigcirc p_{1}, \ldots, \bigcirc p_{n}\right),
$$

2) правило $A / A^{\circ}$ допустимо в $L$.

ДокАЗАТЕльство. 1) Индукция по длине $A$.

Если $A=p_{i}$, то $A^{\circ}=A, \bigcirc A\left(\bigcirc p_{1}, \ldots, \bigcirc p_{n}\right)=\bigcirc^{2} p_{i}$ и можно применить (Sg1). Если $A=\bigcirc_{2} B$ и утверждение верно для $B$, то $A^{\circ}=\bigcirc_{1} B^{\circ}$, и тогда получаем

$$
L \vdash A^{\circ} \equiv \bigcirc_{1} \bigcirc B\left(\bigcirc p_{1}, \ldots, \bigcirc p_{n}\right) .
$$


Последняя формула эквивалентна $\bigcirc_{1} B\left(\bigcirc p_{1}, \ldots, \bigcirc p_{n}\right)$ ввиду $(\mathrm{Sg} 4)$, и далее $\bigcirc \bigcirc_{2} B\left(\bigcirc p_{1}, \ldots, \bigcirc p_{n}\right)=\bigcirc A\left(\bigcirc p_{1}, \ldots, \bigcirc p_{n}\right)\left(\right.$ так как $L \vdash \bigcirc_{1} p \equiv \bigcirc_{2} p$ по (Sg3) и $(\mathrm{Sg} 1))$.

Аналогично, если $A=\bigcirc_{1} B$ и утверждение верно для $B$, то $A^{\circ}=\bigcirc_{2} B^{\circ}$ и имеем

$$
L \vdash A^{\circ} \equiv \bigcirc_{2} \bigcirc B\left(\bigcirc p_{1}, \ldots, \bigcirc p_{n}\right) .
$$

Ввиду (Sg3), последняя формула эквивалентна $\bigcirc \bigcirc_{1} \bigcirc B\left(\bigcirc p_{1}, \ldots, \bigcirc p_{n}\right)$, и далее $\bigcirc \bigcirc_{1} B\left(\bigcirc p_{1}, \ldots, \bigcirc p_{n}\right)=\bigcirc A\left(\bigcirc p_{1}, \ldots, \bigcirc p_{n}\right)$ - по $(\mathrm{Sg} 4)$.

Если $A=\boxminus_{i} B$ и утверждение верно для $B$, то $A^{\circ}=\varpi_{i} B^{\circ}$, и эта формула эквивалентна $\square_{i} \bigcirc B\left(\bigcirc p_{1}, \ldots, \bigcirc p_{n}\right)$, и затем, по ( $\left.\operatorname{Sg} 5\right)$ и $(\operatorname{Sg} 1)$,

$$
\bigcirc \boxminus_{i} B\left(\bigcirc p_{1}, \ldots, \bigcirc p_{n}\right)=\bigcirc A\left(\bigcirc p_{1}, \ldots, \bigcirc p_{n}\right) .
$$

Аналогично рассуждаем в случае $A=\square_{i} B$, используя эквивалентность $\boxminus_{i} \bigcirc p \equiv \bigcirc \square_{i} p$, которая также следует из $(\mathrm{Sg} 5)$ и $(\mathrm{Sg} 1)$.

Остальные случаи $(A=\bigcirc B, A=\perp, A=B \supset C)$ тривиальны.

2) следует из 1).

Лемма доказана.

В частности, если логика $L$ содержит аксиомы (Sg2)-(Sg8), то выводятся и сопряженные их версии $(\mathrm{Sg} 2)^{\circ}-(\mathrm{Sg} 8)^{\circ}$.

\section{4. Аксиоматизация квадратов Сегерберга}

ОПРЕДЕЛЕНИЕ 4.1. Для $n$-модальной логики $\boldsymbol{\Lambda}$ положим

$$
[\boldsymbol{\Lambda}, \boldsymbol{\Lambda}]^{\circledast}:=[\boldsymbol{\Lambda}, \boldsymbol{\Lambda}] * \mathbf{S L}_{3}+\{(\operatorname{Sg} 1), \ldots,(\operatorname{Sg} 8)\} .
$$

Модальные связки этой логики обозначаются так же, как в квадратах Сегерберга.

Из принципа сопряженности следует, что эту аксиоматику можно слегка упростить. Именно, вместо $\mathrm{Com}_{i j}$ (определение 2.22) достаточно добавить только односторонние аксиомы коммутативности

$$
\boxminus_{i} \varpi_{j} p \supset \square_{j} \boxminus_{i} p,
$$

поскольку обратные импликации сопряжены к ним.

Наша ближайшая цель - доказательство теоремы полноты.

Теорема 4.2. Если $\boldsymbol{\Lambda}$ - полная модальная логика хорновского типа, то $\boldsymbol{\Lambda}_{\mathrm{Sg}}^{2}=[\boldsymbol{\Lambda}, \boldsymbol{\Lambda}]^{\circledast}$.

Общий план доказательства - тот же, что и для обычных произведений, и по существу восходит к работе [2]. Однако удобнее излагать доказательство основной леммы (см. ниже) в теоретико-игровых терминах, подобно [4].

ЛЕмма 4.3. Если $\boldsymbol{\Lambda}$ - полная модалъная логика хорновского типа, то логика $[\boldsymbol{\Lambda}, \boldsymbol{\Lambda}]^{\circledast}$ счетно аппроксимируема. 
ДокАЗАТЕльство. Получается из леммы 2.4 , если доказать полноту и $\Delta$-элементарность.

Для доказательства $\Delta$-элементарности заметим, что все аксиомы логики $L:=[\boldsymbol{\Lambda}, \boldsymbol{\Lambda}]^{\circledast}$ элементарны. Действительно, для хорновских формул это следует из определения, а для аксиом $\left[\mathbf{K}_{n}, \mathbf{K}_{n}\right]^{\circledast}-$ как отмечалось, из теоремы Салквиста (это можно проверить и непосредственно; см. лемму 3.3). Для замкнутых формул это получается из определения общезначимости.

Полнота $L$ следует из ее d-упорства. d-упорство получается по лемме 2.11 , если заметить, что $L=\left[\mathbf{K}_{n}, \mathbf{K}_{n}\right]^{\circledast}+\boldsymbol{\Lambda} * \boldsymbol{\Lambda} * \mathbf{K}_{3}$, причем $\left[\mathbf{K}_{n}, \mathbf{K}_{n}\right]^{\circledast}$ d-упорна по теореме Салквиста, $\boldsymbol{\Lambda}$ - по теореме Файна-Ван Бентема как полная $\Delta$-элементарная логика, а $\mathbf{K}_{3}$ - тривиально по определению. Лемма доказана.

Лемма 4.4 (основная лемма). Пусть логика $\boldsymbol{\Lambda}$ - хорновского типа, $\boldsymbol{G} \vDash$ $[\boldsymbol{\Lambda}, \boldsymbol{\Lambda}]^{\circledast}$, причем $G-($ не более чем $)$ счетный конус. Тогда для некоторой шкалы $F \vDash \Lambda$ существует р-морфизм $F_{\mathrm{Sg}}^{2} \rightarrow G$.

ДокАЗАТЕЛЬство будем проводить игровым методом, который излагается вначале для частного случая $\boldsymbol{\Lambda}=\mathbf{K}$. Для построения р-морфизма используем специальную (вообще говоря, бесконечную) игру. В процессе игры отображение строится постепенно на расширяющихся подмножествах нужного квадрата. Мы доказываем, что, при соблюдении некоторых условий, в результате расширений получится квадрат (лемма 4.6), затем - что правильная игра обеспечивает р-морфность (лемма 4.7), и наконец - что выигрышная стратегия существует (лемма 4.8).

Итак, пусть $G=\left(V, X, Y, f_{0}, f_{1}, f_{2}\right) \vDash[\mathbf{K}, \mathbf{K}]^{\circledast}-$ конус с корнем $u_{0}$. Пусть $T_{\omega}=\left(\omega^{*}, \sqsubset\right)$ - стандартное бесконечное интранзитивное иррефлексивное дерево; здесь $\omega^{*}-$ множество всех конечных кортежей натуральных чисел,

$$
\alpha \sqsubset \beta \Longleftrightarrow \exists m \beta=\alpha m .
$$

Далее $\prec$ (соответственно $\preceq$ ) обозначает транзитивное (соответственно рефлексивное транзитивное) замыкание $\sqsubset$.

Для непустого кортежа $\alpha$ мы обозначаем через $|\alpha|$ его длину, через $\alpha_{0}$ его первый член и через $\alpha^{-}$его предшественника (т. е. $\left.\alpha^{-} \sqsubset \alpha\right)$.

Опишем выпрямляющую игру (англ. - rectification game) $\mathrm{RG}(G)$, которая позволит построить требуемый р-морфизм $F_{\mathrm{Sg}}^{2} \rightarrow G$. При этом миры $F$ будут строиться как точки из несвязной суммы двух экземпляров $T_{\omega}$. Для простоты обозначений мы определяем эту сумму следующим образом.

ОПРЕДЕЛЕНИЕ 4.5. $T_{\omega} \sqcup T_{\omega}:=T_{\omega} \backslash U$, где $U-$ множество всех кортежей из $\omega^{*}$, начинающихся с 8 или с 9 .

Легко видеть, что шкала $T_{\omega} \sqcup T_{\omega}$ разбивается на два конуса с корнями 8 и 9 . Соответственно $\left(T_{\omega} \sqcup T_{\omega}\right)^{2}$ разбивается на 4 конуса с корнями $(8,9),(9,8),(8,8)$, $(9,9)$. В шкале $\left(T_{\omega} \sqcup T_{\omega}\right)^{2}$ имеются отношения горизонтальной и вертикальной достижимости; они обозначаются соответственно $\sqsubset_{1}$ и $\sqsubset_{2}$. Обратные к ним отношения горизонталъного и вертикалъного предшествования $\left(\sqsupset_{1}\right.$ и $\left.\sqsupset_{2}\right)$.

Точки вида $(8, \beta)$ и $(9, \beta)$ в шкале $\left(T_{\omega} \sqcup T_{\omega}\right)^{2}$ образуют пару двойников.

В игре $\mathrm{RG}(G)$ участвуют два игрока - $\mathbf{A}$ и $\mathbf{E}$ (для игроков в теоретико-модельных играх в литературе встречаются разнообразные названия: Абеляр 
и Элоиза, Новатор и Консерватор и др.). Позиции в игре $\mathrm{RG}(G)$ перед очередным ходом игрока А называются $(G$ - $)$ сетями. Каждая такая сеть есть отображение $h: H \longrightarrow V$, где $H \subseteq U^{2}$ - конечное множество, причем выполнены следующие условия:

(i) (симметричность) $H$ замкнуто относительно функций $\sigma_{1}, \sigma_{2}, \sigma_{0}$;

(ii) (устойчивостъ) $H$ замкнуто относительно предшествования:

$$
u \in H \&\left(v \sqsubset_{1} u \vee v \sqsubset_{2} u\right) \Rightarrow v \in H ;
$$

(iii) (монотонность) $h$ монотонно как отображение шкал $\left(T_{\omega} \sqcup T_{\omega}\right)_{\mathrm{Sg}}^{2}\lceil H \rightarrow G$.

Начальной позицией в игре является сеть $h_{0}: H_{0} \longrightarrow V$, где $H_{0}=\{(8,9)$, $(9,8),(8,8),(9,9)\}$, а $h_{0}$ переводит эти точки соответственно в $u_{0}, f_{0}\left(u_{0}\right), f_{2}\left(u_{0}\right)$, $f_{1}\left(u_{0}\right)$. Игрок $\mathbf{A}$ начинает игру; затем ходы делаются по очереди. Ходы $\mathbf{A}$ называются запросами. Предполагая, что $k$-й ход сделан $\mathbf{E}$ (или $k=0$ ) и возникла позиция $h_{k}: H_{k} \longrightarrow V$, укажем разрешенные варианты для $(k+1)$-го хода $\mathbf{A}$ и разрешенные ответные ходы $\mathbf{E}$.

1. Поднятие горизонтальной стрелки. Такой ход есть тройка вида $(a, x, y) \in$ $H_{k} \times V^{2}$, где $x=h_{k}(a)$ и $(x, y) \in X$. Ответом должна быть сеть $h_{k+1}: H_{k+1} \longrightarrow$ $V$ такая, что $h_{k} \subseteq h_{k+1}$, в которой

$$
\exists b \in H_{k+1}\left(a \sqsubset{ }_{1} b \& h_{k+1}(b)=y\right)
$$

(т. е. $h_{k+1}$ поднимает стрелку $x X y$ до $a \sqsubset_{1} b$, см. рис. 6 ).

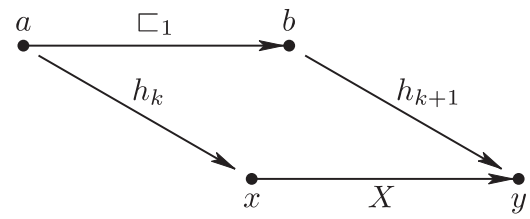

Рис. 6

2. Конвергениия. Тройка $(a, b, c) \in H_{k}^{3}$ такая, что $a \sqsubset_{1} b, a \sqsubset_{2} c$. Ответом должна быть сеть $h_{k+1}: H_{k+1} \longrightarrow V$, где $h_{k} \subseteq h_{k+1}$ и

$$
\exists d \in H_{k+1}\left(b \sqsubset_{2} d \& c \sqsubset_{1} d\right)
$$

(т. е. свойство Чёрча-Россера $\sqsupset_{1} \circ \sqsubset_{2} \subseteq \sqsubset_{2} \circ \sqsupset_{1}$ выполняется для пары $(b, c)$, см. рис. 7).

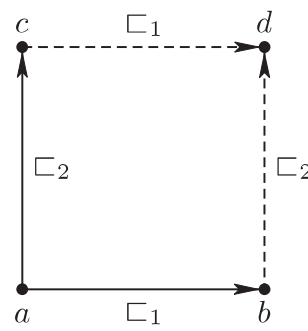

Рис. 7

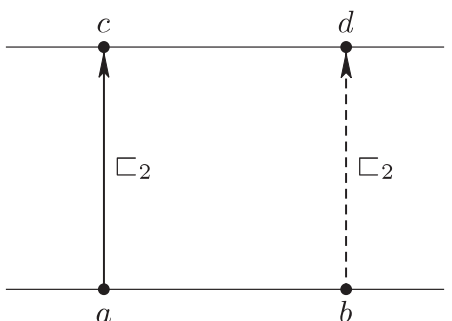

Рис. 8 
3. Выход на горизонталь из двойника. Тройка $(a, b, c) \in H_{k}^{3}$ такая, что $a, b-$ двойники и $a \sqsubset_{2} c$. Ответом должна быть сеть $h_{k+1}: H_{k+1} \longrightarrow V$, где $h_{k} \subseteq h_{k+1}$ и есть выход из $b$ на горизонталь $c$ :

$$
\exists d \in H_{k+1}\left(b \sqsubset_{2} d \& \sigma_{1}(d)=\sigma_{1}(c)\right),
$$

см. рис. 8 .

Имеется тривиальный случай, когда в начальной позиции $\mathbf{A}$ не может сделать ни одного хода: если $X=Y=\varnothing$. В этом случае лемма справедлива, так как $h_{0}$ является нужным р-морфизмом (и $\left.F=(\{8,9\}, \varnothing)\right)$.

В остальных случаях всегда возможен запрос поднятия горизонтальной стрелки. Действительно, рассмотрим множество

$$
h_{0}\left[H_{0}\right]=\left\{u_{0}, f_{0}\left(u_{0}\right), f_{2}\left(u_{0}\right), f_{1}\left(u_{0}\right)\right\} .
$$

Используя (Sg1)-(Sg4), легко проверить, что оно замкнуто относительно $f_{0}$, $f_{1}, f_{2}$. Поэтому одна из точек $h_{0}\left[H_{0}\right]$ имеет потомка по $X$ или $Y$ - иначе бы эти точки составляли конус с $X=Y=\varnothing$. Следовательно, одна из этих точек имеет потомка по $X$, так как

$$
a Y b \Rightarrow f_{0}(a) X f_{0}(b)
$$

по (Sg5) (см. лемму 3.3), а $h_{0}\left[H_{0}\right]$ замкнуто относительно $f_{0}$.

Таким образом, завершенная партия в игре определяется как последовательность разрешенных ходов, которая либо бесконечна, либо конечна и оканчивается ходом $\mathbf{A}$, на который $\mathbf{E}$ не может ответить. В первом случае выигрывает $\mathbf{E}$, во втором - $\mathbf{A}$.

Выигрышная стратегия для $\mathbf{E}$ - это функция, переводящая каждую возможную позицию в игре (которая является сетью) и разрешенный в ней ход $\mathbf{A}$ в ответный ход $\mathbf{E}$, так что любая завершенная партия, использующая эту стратегию, выигрывается $\mathbf{E}$, т. е. бесконечна.

Лемма 4.6. Предположим, что $H \subseteq U^{2}$ обладает следующими свойствами:

(i) (начальные условия) $H_{0} \subseteq H$,

(ii) (устойчивость) Н замкнуто относительно предшествования,

(iii) (симметричность) $Н$ замкнуто относительно функиий $\sigma_{1}, \sigma_{2}, \sigma_{0}$,

(iv) (конвергениия) если $\left(\alpha^{-}, \beta\right) \in H u\left(\alpha, \beta^{-}\right) \in H$, mo $(\alpha, \beta) \in H$,

(v) (единство двойников) если $a, b-$ двойники, то $a \in H \Leftrightarrow b \in H$.

Тогда $H=\left(\operatorname{pr}_{1}[H]\right)^{2}$ u, следователъно, $\left(T_{\omega} \sqcup T_{\omega}\right)_{\mathrm{Sg}}^{2}\left\lceil H=F_{\mathrm{Sg}}^{2}\right.$, где $F=\left(T_{\omega} \sqcup\right.$ $\left.T_{\omega}\right)\left\lceil\operatorname{pr}_{1}[H]\right.$.

Заметим, что свойство (iv) равносильно общезначимости аксиомы CR в шкале $\left(T_{\omega} \sqcup T_{\omega}\right)_{\mathrm{Sg}}^{2}\lceil H$.

ДокАЗАТЕЛЬСтво. Из симметричности $H$ получаем, что $\operatorname{pr}_{1}[H]=\operatorname{pr}_{2}[H]$.

Из устойчивости $H$ получается (по индукции), что $H$ замкнуто относительно "внутренних проекций”:

$$
(\alpha, \beta) \in H \Longrightarrow\left(\alpha_{0}, \beta\right) \in H \&\left(\alpha, \beta_{0}\right) \in H .
$$


С учетом единства двойников, получаем отсюда:

$$
\beta \in \operatorname{pr}_{2}[H] \Longrightarrow(8, \beta) \in H \&(9, \beta) \in H .
$$

Кроме того, $\operatorname{pr}_{1}[H]$ замкнуто относительно предшественников:

$$
\alpha \in \operatorname{pr}_{1}[H]-\{8,9\} \Longrightarrow \alpha^{-} \in \operatorname{pr}_{1}[H] .
$$

В самом деле, если $\alpha \in \operatorname{pr}_{1}[H]-\{8,9\}$, то из $(*)$ следует, что $(\alpha, 8) \in H$ или $(\alpha, 9) \in H$, а тогда $\left(\alpha^{-}, 8\right) \in H$ или $\left(\alpha^{-}, 9\right) \in H$, в силу устойчивости.

Теперь можно проверить, что $\operatorname{pr}_{1}[H] \times \operatorname{pr}_{1}[H] \subseteq H$. Для этого докажем индукцией по $|\alpha|+|\beta|$, что

$$
(\alpha, \beta) \in \operatorname{pr}_{1}[H] \times \operatorname{pr}_{1}[H] \Longrightarrow(\alpha, \beta) \in H .
$$

Если оба кортежа $\alpha, \beta$ - длины 1 (т. е. 8 или 9), то $(\alpha, \beta) \in H_{0} \subseteq H$.

Пусть $(\alpha, \beta) \in \operatorname{pr}_{1}[H] \times \operatorname{pr}_{1}[H]$ и утверждение $(\sharp)$ доказано для всех пар с меньшей суммой длин. Если при этом $\alpha, \beta \notin\{8,9\}$, то по (***) имеем $\alpha^{-}, \beta^{-} \in \operatorname{pr}_{1}[H]$. Тогда по предположению индукции и устойчивости получаем, что $\left(\alpha^{-}, \beta^{-}\right),\left(\alpha^{-}, \beta\right),\left(\alpha, \beta^{-}\right) \in H$. Отсюда $(\alpha, \beta) \in H-$ по свойству конвергенции (iv).

Пусть теперь ровно один из кортежей $\alpha, \beta$ лежит в $\{8,9\}$. Поскольку $H$ симметрично, то, не ограничивая общности, можем считать, что $\beta \notin\{8,9\}$, $\alpha \in\{8,9\}$. Так как $\beta \in \operatorname{pr}_{2}[H]$, то из $(* *)$ следует, что $(8, \beta) \in H \&(9, \beta) \in H$. Поэтому $(\alpha, \beta) \in H$. Лемма доказана.

Лемма 4.7. Если в выпрямляющей игре $\operatorname{RG}(G)$ имеется выигрышная стратегия для $\mathbf{E}$, то для некоторой шкаль $F \subseteq T_{\omega} \sqcup T_{\omega}$ существует р-морфизм $F_{\mathrm{Sg}}^{2} \rightarrow G$.

ДоказАтельство. Расположим все элементы множества $\left(U \times V^{2}\right) \cup U^{3}$ в счетную последовательность: $\mu_{1}, \mu_{2}, \ldots$. Очевидно, что она содержит все потенциальные ходы игрока $\mathbf{A}$ (и много лишних троек).

Как уже отмечалось, в начальной позиции имеется хотя бы один разрешенный ход для $\mathbf{A}$ (поднятие горизонтальной стрелки), который годится для любой позиции. Зафиксируем этот ход как запасной.

Пусть теперь $\mathbf{A}$ делает ходы по следующему правилу: в последовательности $\mu_{1}, \mu_{2}, \ldots$ выбирается первая тройка, которая является разрешенным ходом в данной позиции и которая не использовалась ранее. Если же такой тройки не существует, то А делает запасной ход.

Используя в этой партии выигрышную стратегию, $\mathbf{E}$ строит возрастающую последовательность сетей $h_{n}: H_{n} \longrightarrow V$. Тогда получаем множество $H:=$ $\bigcup_{n} H_{n}$ и отображение $h:=\bigcup_{n} h_{n}$.

Так как все $H_{n}$ замкнуты относительно $\sigma_{0}, \sigma_{1}, \sigma_{2}$, то же верно и для $H$. Устойчивость всех $H_{n}$ также распространяется на $H$.

Рассмотрим подшкалу $\Theta:=\left(T_{\omega} \sqcup T_{\omega}\right)_{\mathrm{Sg}}^{2}\lceil H$. Докажем, что $\Theta-$ квадрат Сегерберга и $h: \Theta \rightarrow G$. Ввиду леммы 4.6 и определения сети, для доказательства "квадратности" $\Theta$ нам достаточно проверить свойство конвергенции и единство двойников. Как мы увидим далее, оба эти свойства получаются из игры.

Монотонность $h$ следует из монотонности всех $h_{n}$. 
Свойство поднятия для функций $f_{0}, f_{1}, f_{2}$ следует из монотонности $h$; для функций монотонность превращается в условие гомоморфности:

$$
h\left(\sigma_{i}(x)\right)=f_{i}(h(x)) .
$$

Свойство поднятия для горизонтальных отношений обеспечивается игрой. Действительно, пусть $a \in H_{n}, h(a)=h_{n}(a)=u, u X v$. Тогда запрос $(a, u, v)$ является допустимым ходом игрока $\mathbf{A}$ в любой позиции с номером $\geqslant n$. Этот запрос есть некоторое $\mu_{k}$. В соответствии с выбором ходов $\mathbf{A}$, он окажется ходом с каким-то номером $l \leqslant n+k$. В самом деле, если этот ход не был сделан ранее, чем на шаге $n$, то он будет сделан не позже, чем на шаге $n+k$, поскольку все допустимые ходы из списка $\mu_{1}, \ldots, \mu_{k-1}$ исчерпаются до шага $n+$ $k$, а запасной ход не делается, пока не сделан допустимый ход $\mu_{k}$. После ответа игрока $\mathbf{E}$ на данный запрос, возникает сеть $h_{l+1}: H_{l+1} \longrightarrow V$, для которой

$$
\exists b \in H_{l+1}\left(a \sqsubset_{1} b \& h_{k+1}(b)=v\right) .
$$

Тогда $h(b)=v, b \in H$, что и требовалось.

Для вертикальных отношений свойство поднятия выполнится само собой. Действительно, пусть $h(a)=u, u Y v$; тогда $h\left(\sigma_{0}(a)\right)=f_{0}(u), f_{0}(u) X f_{0}(v)$ в силу гомоморфности $h$ и $(\mathrm{Sg} 5)$. По тем же причинам (и $(\mathrm{Sg} 1))$

$$
a \sqsubset_{2} \sigma_{0}(b) \& h\left(\sigma_{0}(b)\right)=v \Longleftrightarrow \sigma_{0}(a) \sqsubset_{1} b \& h(b)=f_{0}(v),
$$

и, значит, если горизонтальная стрелка $\left(f_{0}(u), f_{0}(v)\right)$ поднимается до $\left(\sigma_{0}(a), b\right)$, то вертикальная стрелка $(u, v)$ поднимается до $\left(a, \sigma_{0}(b)\right)$.

Сюръективность $h$ следует из свойства поднятия, поскольку $G$ - конус с корнем $u_{0}$, который попадает в образ $h$.

Свойство конвергенции для $\Phi$

$$
\sqsupset_{1} \circ \sqsubset_{2} \subseteq \sqsubset_{2} \circ \sqsupset_{1}
$$

выполнится за счет игры. Действительно, предположим, что $(a, b, c) \in H^{3}, a \sqsubset_{1}$ $b, a \sqsubset_{2} c$. Тогда $(a, b, c) \in H_{n}^{3}$ для некоторого $n$, а потому запрос конвергенции $(a, b, c)$ допустим во всех позициях с номерами $\geqslant n$. Если $(a, b, c)=\mu_{k}$, то этот запрос будет сделан не позже, чем на шаге $n+k,-$ это доказывается так же, как в случае запроса поднятия. После ответа игрока $\mathbf{E}$ на данный запрос возникает сеть $h_{l+1}: H_{l+1} \longrightarrow V$, для которой

$$
\exists d \in H_{l+1}\left(b \sqsubset_{2} d \& c \sqsubset_{1} d\right),
$$

и, значит, свойство конвергенции выполняется в $\Phi$ для пары $(b, c)$.

Попутно отметим, что свойство конвергенции распространяется на пару отношений $\prec_{1}, \sqsubset_{2}\left(\prec_{1}-\right.$ транзитивное замыкание $\left.\sqsubset_{1}\right)$ : по индукции получаем, что

отсюда

$$
\left(\sqsupset_{1}\right)^{n} \circ \sqsubset_{2} \subseteq \sqsubset_{2} \circ\left(\sqsupset_{1}\right)^{n} ;
$$

$$
\succ_{1} \circ \sqsubset_{2} \subseteq \sqsubset_{2} \circ \succ_{1}
$$

Похожее рассуждение применяется и для доказательства единства двойников. Эквивалентность

$$
(8, \beta) \in H \Leftrightarrow(9, \beta) \in H
$$

устанавливаем индукцией по $|\beta|$.

В силу начальных условий, утверждение верно для $|\beta|=1$. 
Пусть (๒) верно для $\beta^{-}$и предположим, не ограничивая общности, что $(8, \beta) \in H$. В силу устойчивости $H$, тогда $\left(8, \beta^{-}\right) \in H$; отсюда $\left(9, \beta^{-}\right) \in H-$ по предположению индукции. Если $a=\left(8, \beta^{-}\right), b=\left(9, \beta^{-}\right), c=(8, \beta)$ лежат в $H_{n}$, то запрос выхода $(a, b, c)$ - допустимый ход $\mathbf{A}$ в любой позиции с номером $\geqslant n$. Пусть $(a, b, c)=\mu_{k}$. Тогда на каком-то шаге $l \leqslant n+k$ этот ход будет сделан. В результате ответа игрока Е получим $(9, \beta) \in H_{l+1}$ (см. рис. 9). Лемма доказана.

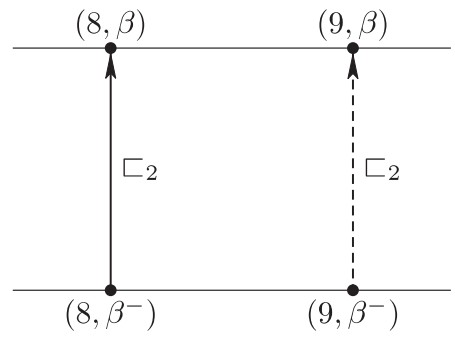

Рис. 9

Лемма 4.8. В игре $\operatorname{RG}(G)$ имеется выигрышная стратегия для $\mathbf{E}$.

ДокАЗАТЕЛьСтво. В позиции $h_{k}$ рассмотрим всевозможные типы ходов (I, II, III) игрока $\mathbf{A}$ и укажем ответные ходы игрока $\mathbf{E}$.

I. ЗАПРОС ПОДНЯТИЯ ГОРИЗОНТАЛЬНОЙ СТРЕЛКИ, т. е. $(a, x, y) \in H_{k} \times V^{2}$, где $x=h_{k}(a) X y$.

Здесь есть тривиальный случай - когда поднятие уже имеется в $H_{k}$ :

$$
\exists b \in H_{k}\left(a \sqsubset{ }_{1} b \& h_{k}(b)=y\right) .
$$

Тогда ответом служит та же сеть, т. е. $h_{k+1}:=h_{k}$.

В нетривиальном случае пусть $a=(\alpha, \beta)$. Так как $H_{k}$ конечно, можно выбрать $\gamma \sqsupset \alpha$ такое, что $\gamma \notin \operatorname{pr}_{1}\left[H_{k}\right]$. Тогда в качестве $b$ берем $(\gamma, \beta)$. Для этого полагаем

$$
H_{k+1}:=H_{k} \cup\{(\varepsilon, \gamma) \mid \varepsilon \preceq \beta \vee \varepsilon \preceq \gamma\} \cup\{(\gamma, \varepsilon) \mid \varepsilon \preceq \beta \vee \varepsilon \preceq \gamma\} .
$$

Так как $\gamma \notin \operatorname{pr}_{1}\left[H_{k}\right]=\operatorname{pr}_{2}\left[H_{k}\right]$, все указанные точки - новые.

Из построения ясно, что $H_{k+1}$ устойчиво: предшественники новой точки $(\varepsilon, \gamma)$ - это новая точка $\left(\varepsilon^{-}, \gamma\right)$ и точка $(\varepsilon, \alpha)$, которая лежит в $H_{k}$ при $\varepsilon \preceq \beta$ или $\varepsilon \preceq \alpha$ (так как $H_{k}$ устойчиво и содержит $(\beta, \alpha)$; кроме того, $(\alpha, \alpha)=\sigma_{2}(\alpha, \beta) \in$ $\left.H_{k}\right)$; предшественники $(\gamma, \gamma)$ - это новые точки $(\alpha, \gamma),(\gamma, \alpha)$, см. рис. 10.

Докажем, что $H_{k+1}$ симметрично. Для этого достаточно проверить действие $\sigma_{0}, \sigma_{1}$ на новых точках: $\sigma_{0}(\varepsilon, \gamma)=(\gamma, \varepsilon) ; \sigma_{1}(\varepsilon, \gamma)=(\gamma, \gamma) \in H_{k+1}$; $\sigma_{1}(\gamma, \varepsilon)=(\varepsilon, \varepsilon)=\sigma_{1}(\alpha, \varepsilon) \in H_{k}$ при $\varepsilon \preceq \beta$ или $\varepsilon \preceq \alpha$, так как $H_{k}$ устойчиво и содержит $(\alpha, \beta)$ и $(\alpha, \alpha)=\sigma_{2}(\alpha, \beta)$; наконец, $\sigma_{1}(\gamma, \gamma)=\sigma_{2}(\gamma, \gamma)=(\gamma, \gamma)$.

Доопределим функцию $h_{k+1}$ в новых точках следующим образом. Полагаем сначала

$$
h_{k+1}(\gamma, \beta):=y, \quad h_{k+1}(\beta, \gamma):=f_{0}(y), \quad h_{k+1}(\gamma, \gamma):=f_{2}(y) .
$$




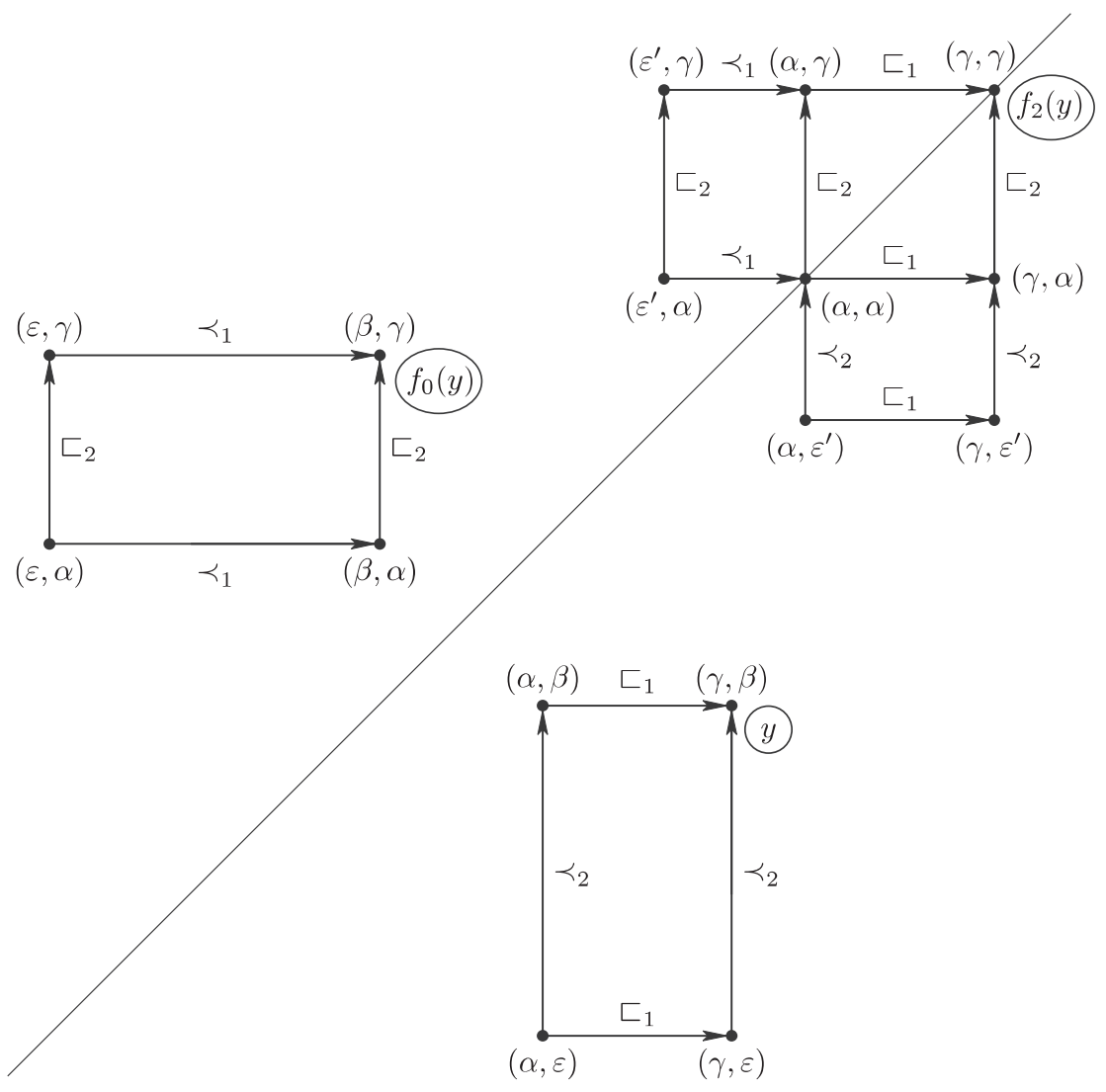

Рис. 10

Чтобы определить $h_{k+1}(\gamma, \varepsilon)$ для всех $\varepsilon \preceq \beta$, действуем по убывающей индукции. Если $|\varepsilon|>1$ и $h_{k+1}(\gamma, \varepsilon)$ уже определено, причем

$$
h_{k}(\alpha, \varepsilon) X h_{k+1}(\gamma, \varepsilon),
$$

то определим $h_{k+1}\left(\gamma, \varepsilon^{-}\right)$. Так как $\varepsilon^{-} \prec \varepsilon \preceq \beta$ и $(\alpha, \beta) \in H_{k}$, то из устойчивости $H_{k}$ получаем, что $(\alpha, \varepsilon),\left(\alpha, \varepsilon^{-}\right) \in H_{k}$ (см. рис. 11). По монотонности $h_{k}$,

$$
h_{k}\left(\alpha, \varepsilon^{-}\right) Y h_{k}(\alpha, \varepsilon) \text {. }
$$

Из (1), (2) и коммутативности $G$ получим $z \in V$ такое, что

$$
h_{k}\left(\alpha, \varepsilon^{-}\right) X z Y h_{k+1}(\gamma, \varepsilon) .
$$

Тогда полагаем $h_{k+1}\left(\gamma, \varepsilon^{-}\right):=z$; таким образом, (1) будет выполнено для $\varepsilon^{-}$:

$$
h_{k}\left(\alpha, \varepsilon^{-}\right) X h_{k+1}\left(\gamma, \varepsilon^{-}\right) .
$$

Кроме того, по определению

$$
h_{k+1}\left(\gamma, \varepsilon^{-}\right) Y h_{k+1}(\gamma, \varepsilon) .
$$




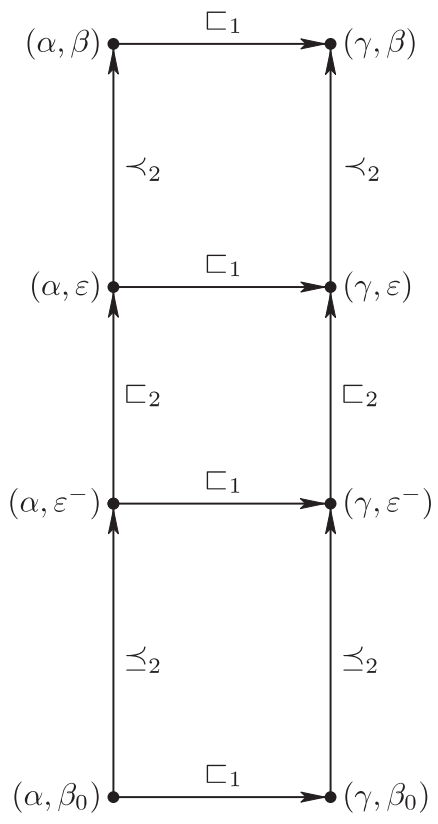

Рис. 11

Если определено $h_{k+1}(\gamma, \varepsilon)$, то определим

$$
h_{k+1}(\varepsilon, \gamma):=f_{0}\left(h_{k+1}(\gamma, \varepsilon)\right) .
$$

Чтобы определить $h_{k+1}(\varepsilon, \gamma), h_{k+1}(\gamma, \varepsilon)$ для всех $\varepsilon \preceq \alpha$, рассмотрим два случая.

Случай 1: $\alpha_{0}=\beta_{0}$, т. е. $\alpha$ и $\beta$ лежат в одном конусе. Пусть $\eta-$ наибольший их общий предок, т.е. $\eta$ - кортеж наибольшей длины в $\{\varepsilon \mid \varepsilon \preceq \alpha \& \varepsilon \preceq \beta\}$. Тогда, если $\eta \preceq \varepsilon \preceq \alpha$, то определяем $h_{k+1}(\gamma, \varepsilon)$ возрастающей индукцией по $|\varepsilon|$ с соблюдением условия (1). Значение $h_{k+1}(\gamma, \eta)$ уже определено, и базис индукции обеспечен.

Если $|\varepsilon|>1$ и $h_{k+1}\left(\gamma, \varepsilon^{-}\right)$определено, то определим $h_{k+1}(\gamma, \varepsilon)$ следующим образом. Из условия (1) для $\varepsilon^{-}$и монотонности $h_{k}$ получаем

$$
h_{k}\left(\alpha, \varepsilon^{-}\right) X h_{k+1}\left(\gamma, \varepsilon^{-}\right), \quad h_{k}\left(\alpha, \varepsilon^{-}\right) Y h_{k}(\alpha, \varepsilon) .
$$

Применив свойство Чёрча-Россера для $G$, найдем $z \in V$ такое, что

$$
h_{k}(\alpha, \varepsilon) X z, \quad h_{k}\left(\gamma, \varepsilon^{-}\right) Y z .
$$

Теперь положим $h_{k+1}(\gamma, \varepsilon):=z$; тогда условия $(1),(3)$ выполнятся для $\varepsilon$.

Затем для всех $\varepsilon \preceq \alpha$ полагаем

$$
h_{k+1}(\varepsilon, \gamma):=f_{0}\left(h_{k+1}(\gamma, \varepsilon)\right) .
$$

Случай 2: $\alpha_{0} \neq \beta_{0}$. Напомним, что по построению

$$
a=(\alpha, \beta) \sqsubset_{1} b=(\gamma, \beta), \quad x=h_{k}(a) X y=h_{k+1}(b), \quad f_{0}(y)=h_{k+1}\left(\sigma_{0}(b)\right) .
$$


Тогда из гомоморфности $h_{k}$ и $(\mathrm{Sg} 5)$ для $G$ имеем

$$
h_{k}\left(\sigma_{0}(a)\right)=f_{0}(x) Y f_{0}(y) .
$$

По построению и $(\mathrm{Sg} 3)$ для $G$ также

$$
h_{k+1}(\gamma, \gamma)=f_{2}(y)=f_{1}\left(f_{0}(y)\right) .
$$

Теперь применим $(\mathrm{Sg} 8)$ для выхода на горизонталь $f_{0}(y)$ из диагональной точки $f_{1}\left(f_{0}(x)\right)=f_{2}(x)$ : найдем $z \in V$ такое, что

$$
f_{2}(x) Y z, \quad f_{1}(z)=f_{2}(y)
$$

Полагаем тогда $h_{k+1}(\alpha, \gamma):=z$. По гомоморфности $h_{k}$ также имеем

$$
f_{2}(x)=h_{k}\left(\sigma_{2}(a)\right)=h_{k}(\alpha, \alpha)
$$

таким образом,

$$
h_{k}(\alpha, \alpha) Y h_{k+1}(\alpha, \gamma)
$$

Кроме того, поскольку $f_{2}(x) Y z$, то по $(\operatorname{Sg} 5)^{\circ}$ для $G$ получаем $z X f_{1}(z)=f_{2}(y)$; следовательно,

$$
h_{k+1}(\alpha, \gamma) X h_{k+1}(\gamma, \gamma)
$$

Теперь можно определить

$$
h_{k+1}(\gamma, \alpha):=f_{0}\left(h_{k+1}(\alpha, \gamma)\right)
$$

при этом из (Sg5) получаем, в частности,

$$
h_{k}(\alpha, \alpha) X h_{k+1}(\gamma, \alpha)
$$

Затем индукцией спуска определяем $h_{k+1}(\gamma, \varepsilon)$ для всех $\varepsilon \preceq \alpha$. Это построение уже приводилось выше; определяем последовательно значения $h_{k+1}$ так, чтобы выполнялись условия монотонности

$$
\begin{array}{r}
h_{k}(\alpha, \varepsilon) X h_{k+1}(\gamma, \varepsilon), \\
h_{k+1}\left(\alpha, \varepsilon^{-}\right) Y h_{k+1}(\gamma, \varepsilon),
\end{array}
$$

см. рис. 12 .

Наконец, полагаем для $\varepsilon \prec \alpha$

$$
h_{k+1}(\varepsilon, \gamma):=f_{0}\left(h_{k+1}(\gamma, \varepsilon)\right)
$$

Гомоморфность $h_{k+1}$ по $\sigma_{0}, \sigma_{1}$ (напомним, что $\sigma_{2}$ через них выражается) надо проверять на всех новых точках $(\gamma, \varepsilon),(\varepsilon, \gamma)$. 


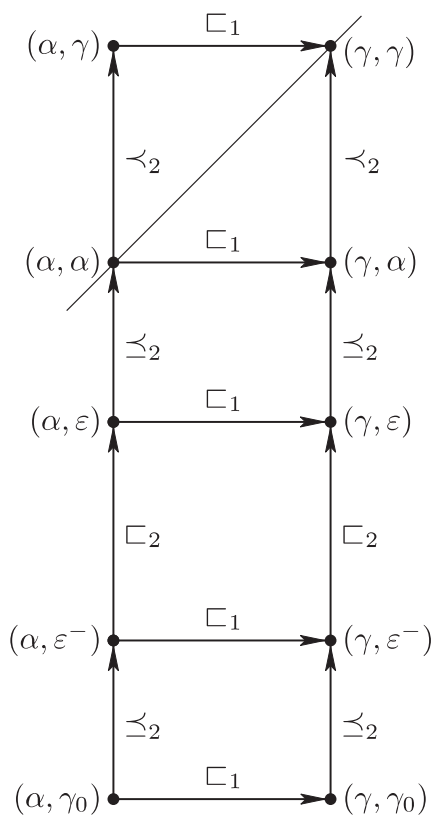

Рис. 12

Для $\sigma_{0}$ все очевидно: при $\varepsilon \preceq \alpha$ или $\varepsilon \preceq \beta$ имеем $h_{k+1}(\varepsilon, \gamma)=f_{0}\left(h_{k+1}(\gamma, \varepsilon)\right)$, по определению и так как $f_{0}$ - инволюция; кроме того, $h_{k+1}(\gamma, \gamma)=f_{2}(y)-$ диагональная точка.

$h_{k+1}\left(\sigma_{1}(\gamma, \gamma)\right)=f_{1}\left(h_{k+1}(\gamma, \gamma)\right)$, так как $f_{2}(y)=f_{1}\left(f_{2}(y)\right)-$ по $(\operatorname{Sg} 3)$ и $(\mathrm{Sg} 2)$.

Рассмотрим $(\gamma, \varepsilon)$ для остальных $\varepsilon$. Так как по (1)

$$
h_{k}(\alpha, \varepsilon) X h_{k+1}(\gamma, \varepsilon) \text {, }
$$

получаем

$$
f_{1}\left(h_{k+1}(\gamma, \varepsilon)\right)=f_{1}\left(h_{k}(\alpha, \varepsilon)\right)
$$

- по (Sg7). Но по гомоморфности $h_{k}$

$$
f_{1}\left(h_{k}(\alpha, \varepsilon)\right)=h_{k}\left(\sigma_{1}(\alpha, \varepsilon)\right)=h_{k}(\varepsilon, \varepsilon)=h_{k+1}(\varepsilon, \varepsilon)=h_{k+1}\left(\sigma_{1}(\gamma, \varepsilon)\right) .
$$

Рассмотрим теперь $(\varepsilon, \gamma)$. Так как

$$
\sigma_{1}(\varepsilon, \gamma)=(\gamma, \gamma), \quad f_{1}\left(h_{k+1}(\varepsilon, \gamma)\right)=f_{1}\left(f_{0}\left(h_{k+1}(\gamma, \varepsilon)\right)\right)=f_{2}\left(h_{k+1}(\gamma, \varepsilon)\right),
$$

нам надо доказать

$$
h_{k+1}(\gamma, \gamma)=f_{2}\left(h_{k+1}(\gamma, \varepsilon)\right) .
$$

При $\varepsilon \preceq \beta$ рассуждаем убывающей индукцией по $|\varepsilon|$. Если $\varepsilon=\beta$, то (5) верно по определению. Шаг индукции сразу получается из $(3)$ и $(\operatorname{Sg} 7)^{\circ}$.

Затем для $\varepsilon \preceq \alpha$ в случае $\alpha_{0}=\beta_{0}$ аналогично рассуждаем возрастающей индукцией по $|\varepsilon|$, начиная с $\varepsilon=\eta$.

При $\varepsilon \preceq \alpha$ в случае $\alpha_{0} \neq \beta_{0}$ сначала рассмотрим $\varepsilon=\alpha$. Согласно (4), имеем $h_{k+1}(\alpha, \gamma) X h_{k+1}(\gamma, \gamma)$, откуда $h_{k+1}(\gamma, \alpha) Y h_{k+1}(\gamma, \gamma)$ по $(\operatorname{Sg} 5)$, и затем применяем $(\operatorname{Sg} 7)^{\circ}$. Далее опять действуем убывающей индукцией по $|\varepsilon|$. 
Наконец, докажем монотонность $h_{k+1}$ по горизонтальным отношениям, т. е. (поскольку $h_{k}$ уже монотонно) - что $h_{k+1}$ сохраняет появившиеся горизонтальные стрелки; тогда монотонность для вертикальных отношений получится по сопряженности. Итак, предположим, что $a \sqsubset_{1} b$, причем одна из точек $a, b$ - новая. Заметим, что если $a-$ новая, то $b \notin H_{k}$, ввиду устойчивости $H_{k}$. Поэтому остаются следующие 3 случая.

1. $a=(\alpha, \varepsilon), b=(\gamma, \varepsilon)$, где $\varepsilon \preceq \alpha$ или $\varepsilon \preceq \beta$. Тогда $h_{k+1}(a) X h_{k+1}(b)$, по свойству (1).

2. $a=\left(\varepsilon^{-}, \gamma\right), b=(\varepsilon, \gamma)$, где $\varepsilon \preceq \alpha$ или $\varepsilon \preceq \beta$. Тогда

$$
f_{0}\left(h_{k+1}(a)\right)=h_{k+1}\left(\sigma_{0}(a)\right) Y h_{k+1}\left(\sigma_{0}(b)\right)=f_{0}\left(h_{k+1}(b)\right)
$$

- по гомоморфности $h_{k+1}$ и свойству (3). Отсюда, по (Sg5),

$$
h_{k+1}(a) X h_{k+1}(b) \text {. }
$$

3. $a=(\alpha, \gamma), b=(\gamma, \gamma)$. Тогда нужно использовать свойство

$$
h_{k+1}(\alpha, \gamma) X h_{k+1}(\gamma, \gamma)
$$

Оно уже доказано для случая, когда $\alpha_{0} \neq \beta_{0}$. В случае $\alpha_{0}=\beta_{0}$ нам уже известно, что

$$
h_{k+1}(\alpha, \alpha) X h_{k+1}(\gamma, \alpha) .
$$

Так как $h_{k+1}(\alpha, \alpha)$ - диагональная точка, то по (Sg6) получаем:

$$
h_{k+1}(\gamma, \alpha) Y f_{2}\left(h_{k+1}(\gamma, \alpha)\right),
$$

откуда по гомоморфности

$$
h_{k+1}(\gamma, \alpha) Y h_{k+1}(\gamma, \gamma)
$$

Применив (Sg5) и гомоморфность, получаем отсюда (6).

II. ЗАПРос КОНВеРГЕНЦИи, т. е. $(a, b, c) \in H_{k}^{3}$, где $a \sqsubset_{1} b, a \sqsubset_{2} c$. В тривиальном случае нужная (и единственная) точка $d$ такая, что $c \sqsubset_{1} d, b \sqsubset_{2} d$, уже имеется в $H_{k}$, и берем $h_{k+1}:=h_{k}$.

Иначе - полагаем

$$
H_{k+1}:=H_{k} \cup\left\{d, \sigma_{0}(d)\right\} .
$$

Заметим, что $\sigma_{1}(d)=\sigma_{1}(c), \sigma_{1}\left(\sigma_{0}(d)\right)=\sigma_{2}(d)=\sigma_{2}(b)$, и потому $H_{k+1}$ симметрично (поскольку $H_{k}$ симметрично).

Устойчивость $H_{k+1}$ следует из устойчивости $H_{k}$ и того, что предшественники $d$ - это $b, c$, а предшественники $\sigma_{0}(d)$ - это $\sigma_{0}(b), \sigma_{0}(c)$.

Из монотонности $h_{k}$ имеем:

$$
h_{k}(a) X h_{k}(b), \quad h_{k}(a) Y h_{k}(c) .
$$

По свойству конвергенции для $G$ найдем тогда $z$ такое, что

$$
h_{k}(c) X z, \quad h_{k}(b) Y z,
$$

и положим

$$
h_{k+1}(d):=z, \quad h_{k+1}\left(\sigma_{0}(d)\right):=f_{0}(z) .
$$


Очевидна гомоморфность $h_{k+1}$ по $\sigma_{0}$ в новых точках $d, \sigma_{0}(d)$.

Проверим гомоморфность по $\sigma_{1}$. Так как $\sigma_{1}(d)=\sigma_{1}(c)$ и $h_{k}$ - гомоморфизм, имеем:

$$
h_{k+1}\left(\sigma_{1}(d)\right)=h_{k}\left(\sigma_{1}(c)\right)=f_{1}\left(h_{k}(c)\right) .
$$

Из $h_{k}(c) X z$ следует $f_{1}\left(h_{k}(c)\right)=f_{1}(z)$ по $(\mathrm{Sg} 7)$, и потому

$$
h_{k+1}\left(\sigma_{1}(d)\right)=f_{1}(z)=f_{1}\left(h_{k+1}(d)\right) .
$$

Аналогично получаем

$$
h_{k+1}\left(\sigma_{1}\left(\sigma_{0}(d)\right)\right)=h_{k+1}\left(\sigma_{2}(d)\right)=f_{2}(z)=f_{1}\left(f_{0}(z)\right)=f_{1}\left(h_{k+1}\left(\sigma_{0}(d)\right)\right) .
$$

Как и выше, монотонность $h_{k+1}$ надо проверить только на новых горизонтальных стрелках. Ввиду устойчивости $H_{k}$, новые стрелки ведут только в новые точки; поэтому надо рассматривать только стрелки $(c, d),\left(\sigma_{0}(b), \sigma_{0}(d)\right)$. По выбору $z=h_{k+1}(d)$ имеем $h_{k+1}(c) X h_{k+1}(d), h_{k+1}(b) Y h_{k+1}(d)$. Из последнего соотношения в силу (Sg5) и гомоморфности получаем

$$
h_{k+1}\left(\sigma_{0}(b)\right) X h_{k+1}\left(\sigma_{0}(d)\right) .
$$

Это доказывает монотонность.

III. ЗАПРОс ВыхОДА, т. е. $(a, b, c) \in H_{k}^{3}$, где $a, b-$ двойники и $a \sqsubset_{2} c$. Как и раньше, если $d$ такое, что

$$
b \sqsubset_{2} d \& \sigma_{1}(d)=\sigma_{1}(c),
$$

уже есть в $H_{k}$, полагаем $h_{k+1}:=h_{k}$. В противном случае, не ограничивая общности, можем считать, что

$$
a=(8, \beta), \quad b=(9, \beta), \quad c=(8, \gamma), \quad \beta \sqsubset \gamma .
$$

Тогда нужное нам $d$ есть $(9, \gamma) \notin H_{k}$. Полагаем тогда

$$
H_{k+1}:=H_{k} \cup\{(9, \gamma),(\gamma, 9)\} .
$$

Симметричность $H_{k+1}$ следует из симметричности $H_{k}$ и того, что $\sigma_{1}(9, \gamma)=$ $(\gamma, \gamma)=\sigma_{1}(c) \in H_{k}, \sigma_{1}(\gamma, 9)=(9,9) \in H_{0}$.

$H_{k+1}$ устойчиво, так как предшественники новых точек - это $(9, \beta),(\beta, 9)$, лежащие в $H_{k}$.

Из симметричности $H_{k}$ имеем $\sigma_{1}(c)=(\gamma, \gamma) \in H_{k}$, и поэтому, ввиду устойчивости $H_{k}, 9 \preceq \gamma$ невозможно. Следовательно, $8 \preceq \gamma$, а тогда и $8 \preceq \beta$. По устойчивости, также имеем $(\beta, \gamma),(\beta, \beta) \in H_{k}$.

Обозначим

$$
x:=h_{k}(\beta, \beta), \quad y:=h_{k}(\beta, \gamma), \quad z:=h_{k}(9, \beta) .
$$

Тогда по монотонности $h_{k}$

$$
x Y y, \quad x=f_{1}(z) .
$$

Применив (Sg8), получаем $t$ такое, что

$$
z Y t, \quad f_{1}(t)=f_{1}(y) .
$$




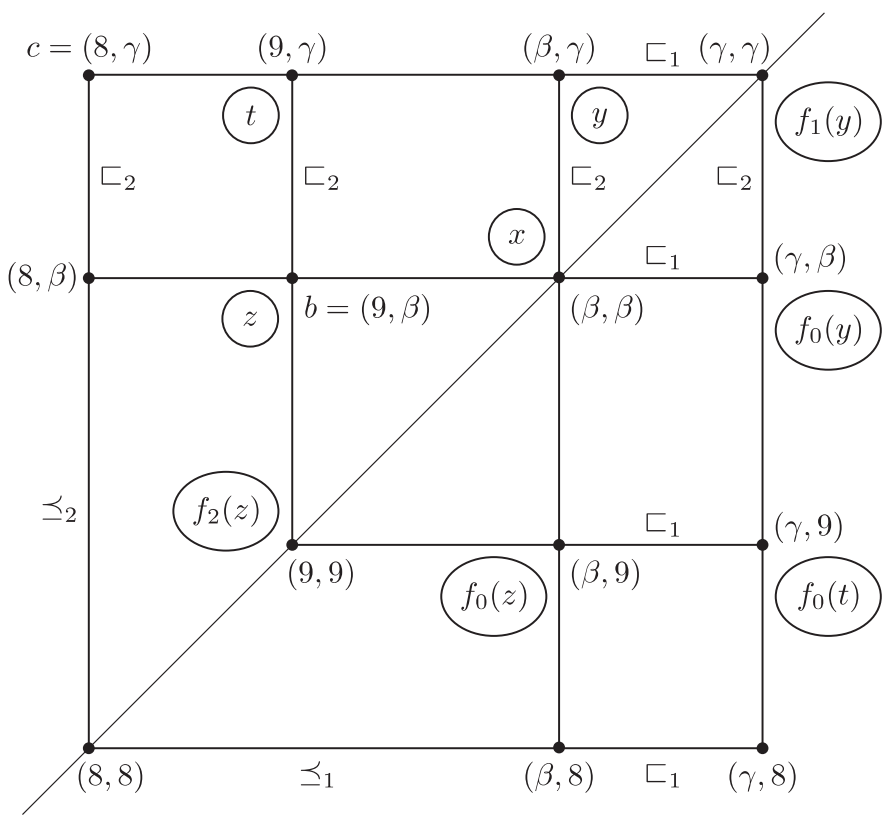

Рис. 13

Тогда доопределим $h_{k+1}$ :

$$
h_{k+1}(9, \gamma):=t, \quad h_{k+1}(\gamma, 9):=f_{0}(t)
$$

(см. рис. 13).

Очевидна гомоморфность $h_{k+1}$ по $\sigma_{0}$. Для проверки гомоморфности по $\sigma_{1}$ в точке $(9, \gamma)$ заметим, что

$$
\begin{aligned}
& h_{k+1}\left(\sigma_{1}(9, \gamma)\right)=h_{k}(\gamma, \gamma)=h_{k}\left(\sigma_{1}(\beta, \gamma)\right), \\
& f_{1}\left(h_{k+1}(9, \gamma)\right)=f_{1}(t)=f_{1}(y)=f_{1}\left(h_{k}(\beta, \gamma)\right),
\end{aligned}
$$

и используем гомоморфность $h_{k}$. А для точки $(\gamma, 9)$ имеем:

$$
h_{k+1}\left(\sigma_{1}(\gamma, 9)\right)=h_{k}(9,9)=h_{k}\left(\sigma_{2}(9, \beta)\right)=f_{2}\left(h_{k}(9, \beta)\right)=f_{2}(z) .
$$

Из $z Y t$ по $(\operatorname{Sg} 7)^{\circ}$ получаем:

$$
f_{2}(z)=f_{2}(t)=f_{1}\left(f_{0}(t)\right)=f_{1}\left(h_{k+1}(\gamma, 9)\right)
$$

откуда следует гомоморфность.

Наконец, докажем монотонность $h_{k+1}$ по горизонтальным стрелкам, где участвуют новые точки. Ввиду устойчивости $H_{k}$, из новых точек нет стрелок, ведущих в $H_{k}$. Поэтому в $H_{k+1}$ появится лишь одна новая горизонтальная стрелка: от $(\beta, 9)$ к $(\gamma, 9)$. Имеем:

$$
h_{k+1}(\beta, 9)=h_{k}(\beta, 9)=f_{0}(z), \quad h_{k+1}(\gamma, 9)=f_{0}(t) .
$$

Но, по построению, $z Y t$, откуда, по $(\mathrm{Sg} 5), f_{0}(z) X f_{0}(t)$, что и требовалось. Это завершает доказательство леммы 4.8. 
Основная лемма для $\boldsymbol{\Lambda}=\mathbf{K}$ сразу следует из лемм $4.7,4.8$.

В случае $\boldsymbol{\Lambda}=\mathbf{K}_{n}$ доказательство сохраняется с некоторыми изменениями, которые мы сейчас опишем.

Деревья теперь рассматриваются как $n$-шкалы. Дерево $T_{\omega}$ заменяется на дерево $T_{\omega, n}$, в котором стрелки раскрашены в $n$ цветов.

ОПРЕДЕЛЕНИЕ 4.9. $T_{\omega, n}:=\left(\omega^{*}, \sqsubset_{1}, \ldots, \sqsubset_{n}\right)$, где

$$
\alpha \sqsubset_{i} \beta:=\exists m(\beta=\alpha m \& m \equiv i(\bmod n)) ;
$$

$T_{\omega, n} \sqcup T_{\omega, n}:=T_{\omega, n}\lceil U(U-$ то же, что в определении 4.5).

Обозначения $\sqsubset_{1}, \sqsubset_{2}$ ранее использовались в другом смысле, но это не должно вызвать путаницы, так как горизонтальные и вертикальные отношения в квадрате $\left(T_{\omega, n} \sqcup T_{\omega, n}\right)^{2}$ будут обозначаться соответственно $\sqsubset_{11}, \ldots, \sqsubset_{n 1}$ и $\sqsubset_{12}$, $\ldots, \sqsubset_{n 2}$.

Пусть исходная шкала есть

$$
G=\left(V, X_{1}, \ldots, X_{n}, Y_{1}, \ldots, Y_{n}, f_{0}, f_{1}, f_{2}\right) \vDash\left[\mathbf{K}_{n}, \mathbf{K}_{n}\right]^{\circledast} .
$$

Определение сети по существу не меняется; условие устойчивости записывается теперь в виде

$$
u \in H \& \exists i\left(v \sqsubset_{i 1} u \vee v \sqsubset_{i 2} u\right) \Longrightarrow v \in H .
$$

В игре $\operatorname{RG}(G)$ имеются те же типы ходов, что и раньше, но с учетом нескольких отношений достижимости.

1. Запрос поднятия горизонтальной стрелки. Это четверка вида $(a, x, y, i) \in$ $H_{k} \times V^{2} \times\{1, \ldots, n\}$, где $x=h_{k}(a)$ и $(x, y) \in X_{i}$. Ответом должна быть сеть $h_{k+1}: H_{k+1} \longrightarrow V$, где $h_{k} \subseteq h_{k+1}$ и

$$
\exists b \in H_{k+1}\left(a \sqsubset_{i 1} b \& h_{k+1}(b)=y\right) .
$$

2. Запрос конвергениии. Пятерка $(a, b, c, i, j) \in H_{k}^{3} \times\{1, \ldots, n\}^{2}$ такая, что $a \sqsubset_{i 1} b, a \sqsubset_{j 2} c$. Ответом должна быть сеть $h_{k+1}: H_{k+1} \longrightarrow V$, где $h_{k} \subseteq h_{k+1}$ и

$$
\exists d \in H_{k+1}\left(b \sqsubset_{j 2} d \& c \sqsubset_{i 1} d\right) .
$$

3. Запрос выхода на горизонталь из двойника. Тройка $(a, b, c, i) \in H_{k}^{3}$ такая, что $a, b$ - двойники и $a \sqsubset_{i 2} c$. Ответом должна быть сеть $h_{k+1}: H_{k+1} \longrightarrow V$, где $h_{k} \subseteq h_{k+1}$ и

$$
\exists d \in H_{k+1}\left(b \sqsubset_{i 2} d \& \sigma_{1}(d)=\sigma_{1}(c)\right) .
$$

Основная лемма справедлива в тривиальной ситуации, когда все отношения $X_{i}, Y_{i}$ - пустые. В остальных случаях возможен запрос поднятия горизонтальной стрелки; это доказывается, как и раньше, с использованием (Sg1)-(Sg5).

В формулировке леммы $4.6 T_{\omega}$ заменяется на $T_{\omega, n}$, а условие конвергенции (iv) становится таким:

$$
\left(T_{\omega, n} \sqcup T_{\omega, n}\right)_{\mathrm{Sg}}^{2}\left\lceil H \vDash \mathrm{CR}_{i, j} \quad \text { для } \quad i, j \in\{1, \ldots, n\} .\right.
$$

Доказательство этой леммы сохраняется.

В формулировке леммы 4.7 также $T_{\omega}$ заменяется на $T_{\omega, n}$. В доказательство вносятся следующие изменения. 
Последовательность $\mu_{n}$ теперь составлена из элементов множества $\left(H_{k} \times\right.$ $\left.V^{2} \times\{1, \ldots, n\}\right) \cup\left(H_{k}^{3} \times\{1, \ldots, n\}^{2}\right) \cup H_{k}^{3}$.

Свойство поднятия нужно проверять для всех горизонтальных отношений. Если $a \in H_{n}, h(a)=h_{n}(a)=u X_{i} v$, то запрос $(a, u, v, i)-$ допустимый ход $\mathbf{A}$ в любой позиции с номером $\geqslant n$ и он делается на шаге $l \leqslant n+k$. Ответ $\mathbf{E}$ дает нужное поднятие.

Свойство поднятия для вертикальных отношений следует по-прежнему из (Sg5).

Для проверки свойства конвергенции

$$
\sqsupset_{i 1} \circ \sqsubset_{j 2} \subseteq \sqsubset_{j 2} \circ \sqsupset_{i 1}
$$

предположим, что $(a, b, c) \in H^{3}, a \sqsubset_{i 1} b, a \sqsubset_{j 2} c$. Тогда $(a, b, c) \in H_{n}^{3}$ для некоторого $n$, а запрос конвергенции $(a, b, c, i, j)$ допустим во всех позициях с номерами $\geqslant n$. После ответа $\mathbf{E}$ получаем сеть $h_{l+1}: H_{l+1} \longrightarrow V$, для которой

$$
\exists d \in H_{l+1}\left(b \sqsubset_{j 2} d \& c \sqsubset_{i 1} d\right) .
$$

При проверке единства двойников на шаге индукции имеем $a=\left(8, \beta^{-}\right), b=$ $\left(9, \beta^{-}\right), c=(8, \beta)$. Если при этом $\beta^{-} \sqsubset_{i} \beta$, то нужно использовать запрос выхода $(a, b, c, i)$.

Наконец, отметим отличия в доказательстве леммы 4.8.

I. Запрос поднятия горизонтальной стрелки имеет вид $(a, x, y, i)$, где $x=$ $h_{k}(a) X_{i} y$. В нетривиальном случае для $a=(\alpha, \beta)$ выбираем $\gamma \sqsupset_{i} \alpha$ такое, что $\gamma \notin \operatorname{pr}_{1}\left[H_{k}\right]$, и полагаем, как и раньше,

$$
\begin{gathered}
H_{k+1}:=H_{k} \cup\{(\varepsilon, \gamma) \mid \varepsilon \preceq \beta \vee \varepsilon \preceq \gamma\} \cup\{(\gamma, \varepsilon) \mid \varepsilon \preceq \beta \vee \varepsilon \preceq \gamma\}, \\
h_{k+1}(\gamma, \beta):=y, \quad h_{k+1}(\beta, \gamma):=f_{0}(y), \quad h_{k+1}(\gamma, \gamma):=f_{2}(y) .
\end{gathered}
$$

Далее по убывающей индукции определяем $h_{k+1}(\gamma, \varepsilon)$ для $\varepsilon \preceq \beta$ с соблюдением свойства

$$
h_{k}(\alpha, \varepsilon) X_{i} h_{k+1}(\gamma, \varepsilon) .
$$

А именно, если $h_{k+1}(\gamma, \varepsilon)$ определено, $|\varepsilon|>1$ и $\varepsilon^{-} \sqsubset j \varepsilon$, то, по монотонности $h_{k}$,

$$
h_{k}\left(\alpha, \varepsilon^{-}\right) Y_{j} h_{k}(\alpha, \varepsilon) .
$$

По коммутативности $G$, находим $z$ такое, что

$$
h_{k}\left(\alpha, \varepsilon^{-}\right) X_{i} z Y_{j} h_{k+1}(\gamma, \varepsilon),
$$

и полагаем $h_{k+1}\left(\gamma, \varepsilon^{-}\right):=z$. Тогда (1) верно для $\varepsilon^{-}$и, кроме того,

$$
h_{k+1}\left(\gamma, \varepsilon^{-}\right) Y_{j} h_{k+1}(\gamma, \varepsilon) \text {. }
$$

Затем в случае $1\left(\alpha_{0}=\beta_{0}\right)$ определяем $h_{k+1}(\gamma, \varepsilon)$ для $\eta \preceq \varepsilon \preceq \alpha$ возрастающей индукцией по $|\varepsilon|$, с соблюдением (1). А именно, если $|\varepsilon|>1, h_{k+1}\left(\gamma, \varepsilon^{-}\right)$ определено и $\varepsilon^{-} \sqsubset_{j} \varepsilon$, то из $(1)$ для $\varepsilon^{-}$и монотонности $h_{k}$ имеем

$$
h_{k}\left(\alpha, \varepsilon^{-}\right) X_{i} h_{k+1}\left(\gamma, \varepsilon^{-}\right), \quad h_{k}\left(\alpha, \varepsilon^{-}\right) Y_{j} h_{k}(\alpha, \varepsilon) .
$$

Тогда, по аксиоме $\left(\mathrm{CR}_{i j}\right)$, найдем $z \in V$ такое, что

$$
h_{k}(\alpha, \varepsilon) X_{i} z, \quad h_{k}\left(\gamma, \varepsilon^{-}\right) Y_{j} z,
$$

и положим $h_{k+1}(\gamma, \varepsilon):=z$. 
В случае $2\left(\alpha_{0} \neq \beta_{0}\right)$ заметим, что

$$
a=(\alpha, \beta) \sqsubset_{i} b=(\gamma, \beta), \quad x=h_{k}(a) X_{i} y=h_{k+1}(b),
$$

откуда, по гомоморфности $h_{k}$ и $(\mathrm{Sg} 5)$,

$$
h_{k}\left(\sigma_{0}(a)\right)=f_{0}(x) Y_{i} f_{0}(y) .
$$

По построению и (Sg3)

$$
h_{k+1}(\gamma, \gamma)=f_{2}(y)=f_{1}\left(f_{0}(y)\right) .
$$

Отсюда по (Sg8) найдем $z \in V$ такое, что

$$
f_{2}(x)=f_{1}\left(f_{0}(x)\right) Y_{i} z, \quad f_{1}(z)=f_{2}(y),
$$

и положим $h_{k+1}(\alpha, \gamma):=z$. Тогда

$$
h_{k}(\alpha, \alpha) Y_{i} h_{k+1}(\alpha, \gamma) \text {. }
$$

Далее, из $f_{2}(x) Y_{i} z$ и $(\mathrm{Sg} 5)^{\circ}$ получаем $z X_{i} f_{1}(z)=f_{2}(y)$; следовательно,

$$
h_{k+1}(\alpha, \gamma) X_{i} h_{k+1}(\gamma, \gamma)
$$

Затем определяем

$$
h_{k+1}(\gamma, \alpha):=f_{0}\left(h_{k+1}(\alpha, \gamma)\right)
$$

а из (Sg5) получаем

$$
h_{k}(\alpha, \alpha) X_{i} h_{k+1}(\gamma, \alpha) .
$$

После этого индукцией спуска определяется $h_{k+1}(\gamma, \varepsilon)$ для $\varepsilon \preceq \alpha$ с соблюдением условий

$$
h_{k}(\alpha, \varepsilon) X_{i} h_{k+1}(\gamma, \varepsilon)
$$

и

$$
h_{k+1}\left(\alpha, \varepsilon^{-}\right) Y_{j} h_{k+1}(\gamma, \varepsilon)
$$

при $\varepsilon^{-} \sqsubset_{j} \varepsilon$. И затем для $\varepsilon \prec \alpha$ определяется

$$
h_{k+1}(\varepsilon, \gamma):=f_{0}\left(h_{k+1}(\gamma, \varepsilon)\right) .
$$

Гомоморфность $h_{k+1}$ по $\sigma_{0}, \sigma_{1}$ проверяется аналогично случаю К. Заметим, что из

$$
h_{k}(\alpha, \varepsilon) X_{i} h_{k+1}(\gamma, \varepsilon)
$$

по (Sg7) по-прежнему следует

$$
f_{1}\left(h_{k+1}(\gamma, \varepsilon)\right)=f_{1}\left(h_{k}(\alpha, \varepsilon)\right) .
$$

При проверке монотонности по горизонтальным отношениям разбираются все те же 3 случая.

1. $a=(\alpha, \varepsilon), b=(\gamma, \varepsilon)$, где $\varepsilon \preceq \alpha$ или $\varepsilon \preceq \beta$. Тогда $h_{k+1}(a) X_{i} h_{k+1}(b)$, по (1).

2. $a=\left(\varepsilon^{-}, \gamma\right), b=(\varepsilon, \gamma)$, где $\varepsilon \preceq \alpha$ или $\varepsilon \preceq \beta, \varepsilon^{-} \sqsubset_{j} \varepsilon$. Тогда

$$
f_{0}\left(h_{k+1}(a)\right)=h_{k+1}\left(\sigma_{0}(a)\right) Y_{j} h_{k+1}\left(\sigma_{0}(b)\right)=f_{0}\left(h_{k+1}(b)\right)
$$

- по гомоморфности $h_{k+1}$ и $(3)$, откуда, по $(\mathrm{Sg} 5)$,

$$
h_{k+1}(a) X_{j} h_{k+1}(b) \text {. }
$$


3. $a=(\alpha, \gamma), b=(\gamma, \gamma)$. Здесь нужно доказывать

$$
h_{k+1}(\alpha, \gamma) X_{i} h_{k+1}(\gamma, \gamma)
$$

В случае $\alpha_{0}=\beta_{0}$ уже известно, что

$$
h_{k+1}(\alpha, \alpha) X_{i} h_{k+1}(\gamma, \alpha),
$$

откуда по (Sg6) и гомоморфности

$$
h_{k+1}(\gamma, \alpha) Y_{i} f_{2}\left(h_{k+1}(\gamma, \alpha)\right)=h_{k+1}(\gamma, \gamma) .
$$

Из (Sg5) и гомоморфности теперь следует (4).

II. Рассмотрим запрос конвергенции $(a, b, c, i, j) \in H_{k}^{3}$, где $a \sqsubset_{i 1} b, a \sqsubset_{j 2} c$. Если нужной точки $d$ нет в $H_{k}$, полагаем

$$
H_{k+1}:=H_{k} \cup\left\{d, \sigma_{0}(d)\right\} .
$$

Тогда, по монотонности $h_{k}$,

$$
h_{k}(a) X_{i} h_{k}(b), \quad h_{k}(a) Y_{j} h_{k}(c),
$$

а по свойству конвергенции для $G$ найдем $z$ такое, что

$$
h_{k}(c) X_{i} z, \quad h_{k}(b) Y_{j} z,
$$

и положим

$$
h_{k+1}(d):=z, \quad h_{k+1}\left(\sigma_{0}(d)\right):=f_{0}(z) .
$$

Для доказательства монотонности используем $h_{k}(c) X_{i} h_{k+1}(d), h_{k}(b) Y_{j} h_{k+1}(d)$ и

$$
h_{k}\left(\sigma_{0}(b)\right) X_{j} h_{k+1}\left(\sigma_{0}(d)\right)
$$

(что получается по $(\mathrm{Sg} 5))$.

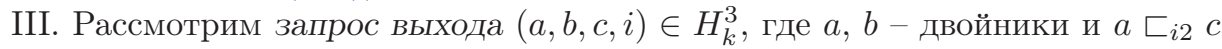
и искомой точки нет в $H_{k}$. Предположим, что

$$
a=(8, \beta), \quad b=(9, \beta), \quad c=(8, \gamma), \quad \beta \sqsubset_{i} \gamma .
$$

Полагаем

$$
H_{k+1}:=H_{k} \cup\{(9, \gamma),(\gamma, 9)\}
$$

Пусть

$$
x:=h_{k}(\beta, \beta), \quad y:=h_{k}(\beta, \gamma), \quad z:=h_{k}(9, \beta) .
$$

Тогда по монотонности $h_{k}$

$$
x Y_{i} y, \quad x=f_{1}(z) .
$$

Используя (Sg8), найдем $t$ такое, что

$$
z Y_{i} t, \quad f_{1}(t)=f_{1}(y),
$$

и определим

$$
h_{k+1}(9, \gamma):=t, \quad h_{k+1}(\gamma, 9):=f_{0}(t) .
$$

6 УМН, т. 67, вып. 4 
Монотонность $h_{k+1}$ проверяется так же, как раньше; заметим только, что из $z Y_{i} t$ следует $f_{2}(z)=f_{2}(t)\left(\right.$ по $\left.(\operatorname{Sg} 7)^{\circ}\right)$ и $f_{0}(z) X_{i} f_{0}(t)($ по $(\operatorname{Sg} 5))$.

Наконец, рассмотрим общий случай, когда $\boldsymbol{\Lambda}$ - произвольная $n$-модальная логика хорновского типа. Пусть

$$
G=\left(V, X_{1}, \ldots, X_{n}, Y_{1}, \ldots, Y_{n}, f_{0}, f_{1}, f_{2}\right) \vDash[\boldsymbol{\Lambda}, \boldsymbol{\Lambda}]^{\circledast}
$$

тогда и $G \vDash\left[\mathbf{K}_{n}, \mathbf{K}_{n}\right]^{\circledast}$, а потому, как уже доказано, существует $h: F_{\mathrm{Sg}}^{2} \rightarrow$ $G$ для некоторой $n$-шкалы $F$. Представим нашу логику $\boldsymbol{\Lambda}$ как $L_{1}+L_{2}$, где аксиомы $L_{1}$ хорновские, а аксиомы $L_{2}$ замкнуты. Тогда $\mathbf{V}\left(L_{1}\right)=\operatorname{Mod}(\Gamma)$, где $\Gamma$ - хорновская теория. Построим хорновское замыкание $F_{\Gamma}^{+}=\left(W, \sqsubset_{1}^{+}, \ldots, \sqsubset_{n}^{+}\right)$ и докажем, что $h:\left(F_{\Gamma}^{+}\right)_{\mathrm{Sg}}^{2} \rightarrow G$.

Гомоморфность $h$ по $\sigma_{0}, \sigma_{1}, \sigma_{2}$ уже известна. Проверим монотонность по новым горизонтальным стрелкам. Заметим, что, в силу монотонности $h$ по ${ }_{i 1}$, для каждого $\beta$ сквозное отображение $h_{\beta}: \alpha \mapsto(\alpha, \beta) \mapsto h(\alpha, \beta)$ монотонно по всем $\sqsubset{ }_{i}$. Заметим также, что $G \vDash \boldsymbol{\Lambda} * \boldsymbol{\Lambda} * \mathbf{K}_{3}$, и потому $\left(V, X_{1}, \ldots, X_{n}\right) \vDash \boldsymbol{\Lambda}$, а значит, и $\left(V, X_{1}, \ldots, X_{n}\right) \vDash \Gamma$. Следовательно, по лемме $2.26, h_{\beta}$ монотонно и как отображение $F_{\Gamma}^{+} \rightarrow\left(V, X_{1}, \ldots, X_{n}\right)$. Таким образом, $\alpha \sqsubset_{i}^{+} \gamma \Rightarrow h(\alpha, \beta) X_{i} h(\gamma, \beta)$, т. е. $h$ монотонно по всем горизонтальным отношениям $\sqsubset_{i 1}^{+}$.

Монотонность по вертикальным отношениям доказывается аналогично.

Из свойства поднятия $h$ по исходным отношениям $\sqsubset_{i 1}, \sqsubset_{i 1}$ следует свойство поднятия по содержащим их новым отношениям $\sqsubset_{i 1}^{+}, \sqsubset_{i 2}^{+}$. Итак, получаем $h:\left(F_{\Gamma}^{+}\right)_{\mathrm{Sg}}^{2} \rightarrow G$.

Далее, $h:\left(W^{2}, \sqsubset_{11}^{+}, \ldots, \sqsubset_{n 1}^{+}\right) \rightarrow\left(V, X_{1}, \ldots, X_{n}\right)$, причем $\left(V, X_{1}, \ldots, X_{n}\right) \vDash L_{2}$ и аксиомы $L_{2}$ замкнуты, поэтому, по лемме $2.16,\left(W^{2}, \sqsubset_{11}^{+}, \ldots, \sqsubset_{n 1}^{+}\right) \vDash L_{2}$. Очевидно, что проекция на первый сомножитель $(\alpha, \beta) \mapsto \alpha-$ это р-морфизм $\left(W^{2}, \sqsubset_{11}^{+}, \ldots, \sqsubset_{n 1}^{+}\right) \rightarrow F_{\Gamma}^{+}$, откуда $F_{\Gamma}^{+} \vDash L_{2}$, и в итоге $F_{\Gamma}^{+} \vDash \boldsymbol{\Lambda}$.

ДокАЗАТЕЛЬСтво теоремы 4.2. Так как любая шкала вида $F^{2}$ удовлетворяет аксиомам коммутирования и конвергенции, то $[\boldsymbol{\Lambda}, \boldsymbol{\Lambda}]^{2} \subseteq \boldsymbol{\Lambda}^{2}$, включение же $[\boldsymbol{\Lambda}, \boldsymbol{\Lambda}] \circledast \boldsymbol{\Lambda}_{\mathrm{Sg}}^{2}$ следует из леммы 3.3.

Для доказательства обратного включения предположим, что $[\boldsymbol{\Lambda}, \boldsymbol{\Lambda}]^{\circledast} \nvdash A$. По лемме 4.3 и лемме о конусах существует не более чем счетный конус $G$ такой, что $G \vDash[\boldsymbol{\Lambda}, \boldsymbol{\Lambda}]^{\circledast}$ и $G \not \models A$. По леммам 4.4 и 2.16 тогда $F_{\mathrm{Sg}}^{2} \not \models A$ и $F \vDash \boldsymbol{\Lambda}$ для некоторой шкалы $F$. Но это и означает, что $\boldsymbol{\Lambda}_{\mathrm{Sg}}^{2} \nvdash A$. Теорема доказана.

Сделаем здесь еще одно замечание, полезное для дальнейшего.

Лемма 4.10. Пусть $\boldsymbol{\Lambda}$ - логика из теоремы 4.2, Г- соответствующая хорновская теория, $A$ - бормула в языке $\boldsymbol{\Lambda}$. Если $\boldsymbol{\Lambda}_{\mathrm{Sg}}^{2} \nvdash A$, то существует шкала $F \subseteq T_{\omega, n}$ такая, ито $\left(F_{\Gamma}^{+}\right)_{\mathrm{Sg}}^{2},(8,9) \not \models A$.

ДокАЗАТЕЛЬство. В доказательстве основной леммы построен р-морфизм $h:\left(F_{\Gamma}^{+}\right)_{\mathrm{Sg}}^{2} \rightarrow G$, где $G, u_{0} \not \models A$ и $h(8,9)=u_{0}$. Отсюда $\left(F_{\Gamma}^{+}\right)_{\mathrm{Sg}}^{2},(8,9) \not \models A$ по лемме о р-морфизме. 


\section{5. О квадратах деревьев}

В этом разделе мы усилим теорему полноты 4.2 для некоторых логик. Напомним, что в доказательстве леммы 4.4 строится р-морфизм $F_{\mathrm{Sg}}^{2} \rightarrow G$ для шкалы $F$, представляющей собой хорновское замыкание леса из двух деревьев. Поэтому $\boldsymbol{\Lambda}_{\mathrm{Sg}}^{2}$ есть логика квадратов Сегерберга таких несвязных шкал. Покажем, что в ряде случаев можно ограничиться лишь деревьями.

ОПРЕДЕЛЕНИЕ 5.1. Стандартным $n$-деревом называется устойчивая подшкала $T_{\omega, n}$, т. е. ограничение $T_{\omega, n}$ на устойчивое подмножество. $n$-деревом называется шкала, изоморфная стандартному $n$-дереву.

Теорема 5.2. Пусть $\boldsymbol{\Lambda}=\mathbf{K}_{n}+\Sigma_{1}+\Sigma_{2}$, где формуль из $\Sigma_{1}$ - псевдотранзитивные вида $\square_{k} p \supset \square_{\alpha} p$, а формулы из $\Sigma_{2}-$ замкнутые вида $\diamond_{i} \top$. Пусть

$$
\mathscr{C}_{\boldsymbol{\Lambda}}:=\left\{F_{\Gamma}^{+} \mid F-n \text {-дерево, } F \vDash \Sigma_{2}\right\},
$$

где Г - хорновская теория, соответствующая $\Sigma_{1}$. Тогда

$$
\mathbf{\Lambda}_{\mathrm{Sg}}^{2}=\mathbf{L}\left(\left\{G_{\mathrm{Sg}}^{2} \mid G \in \mathscr{C}_{\boldsymbol{\Lambda}}\right\}\right) .
$$

ДоказАтельство. Сначала покажем, что $\boldsymbol{\Lambda}_{\mathrm{Sg}}^{2} \subseteq \mathbf{L}\left(\left\{G_{\mathrm{Sg}}^{2} \mid G \in \mathscr{C}_{\mathbf{\Lambda}}\right\}\right)$. Действительно, если $F \vDash \Sigma_{2}$, то и $F_{\Gamma}^{+} \vDash \Sigma_{2}$, так как хорновское замыкание получено добавлением новых стрелок, а потому сохраняет сериальность отношения $R_{i}$. По определению хорновского замыкания, $F_{\Gamma}^{+} \vDash \Sigma_{1}$. Значит, $G \vDash \Lambda$ для всех $G \in \mathscr{C}_{\boldsymbol{\Lambda}}$, откуда и следует нужное включение.

Доказательство обратного включения начнем с простейшего случая, когда $\boldsymbol{\Lambda}=\mathbf{K}_{n}$. Пусть $A \notin \boldsymbol{\Lambda}_{\mathrm{Sg}}^{2}$. Как показывает доказательство теоремы 4.2 , найдутся 1 -деревья $F_{1}$ и $F_{2}$ (с корнями 8 и 9 соответственно), для которых $\left(F_{1} \sqcup F_{2}\right)_{\mathrm{Sg}}^{2} \not \models A$. Пусть дерево $E \subseteq T_{\omega, n}$ получено добавлением корня $\lambda$ (пустого кортежа) к лесу $F:=F_{1} \sqcup F_{2}$; очевидно, что $E \in \mathscr{C}_{\boldsymbol{\Lambda}}$. Тогда $F$ - коническая подшкала в $E$, следовательно, $F_{\mathrm{Sg}}^{2}$ - коническая подшкала в $E_{\mathrm{Sg}}^{2}$. Отсюда, по лемме о конусах, $E_{\mathrm{Sg}}^{2} \not \models A$, что и требовалось.

Рассмотрим теперь случай, когда $\Sigma_{1}=\varnothing, \Sigma_{2} \neq \varnothing$. Пусть дерево $E \subseteq$ $T_{\omega, n}$ получено добавлением $n-2$ изоморфных копий $F_{2}^{(1)}, \ldots, F_{2}^{(n-2)}$ дерева $F_{2}$ с корнями $10, \ldots, n+7$, а также корня $\lambda$ к лесу $F$ (см. рис. 14$)$.

Более формально:

$$
F_{2}^{(i)}=\left\{\left(9+i, j_{1}, \ldots, j_{k}\right) \mid\left(9, j_{1}, \ldots, j_{k}\right) \in F_{2}\right\} .
$$

Тогда сериальность $\sqsubset j$ распространится из $F$ на $E$, так как $\lambda \sqsubset_{j} m$ для какого-то $m \equiv j(\bmod n)$ на отрезке $[8, n+7]$, а сериальность в $F_{2}^{(i)}$ эквивалентна сериальности в $F_{2}$. Таким образом, $E \in \mathscr{C}_{\boldsymbol{\Lambda}}$. Так как $F-$ коническая подшкала в $E$, далее можно рассуждать как в случае $\boldsymbol{\Lambda}=\mathbf{K}_{n}$.

В общем случае, согласно доказательству теоремы 4.2 , найдутся деревья $F_{1}$ и $F_{2}$ такие, что $\left(F_{\Gamma}^{+}\right)_{\mathrm{Sg}}^{2} \not \models A$, где $F:=F_{1} \sqcup F_{2}$ (ради удобства далее мы опускаем индекс $\Gamma$ ). Тогда мы сначала построим дерево $E$, как только что было описано, а затем - его хорновское замыкание $E^{+}$. Ясно, что $E^{+} \in \mathscr{C}_{\boldsymbol{\Lambda}}$, и можно повторить предыдущие рассуждения, если проверить, что $F^{+}-$коническая 


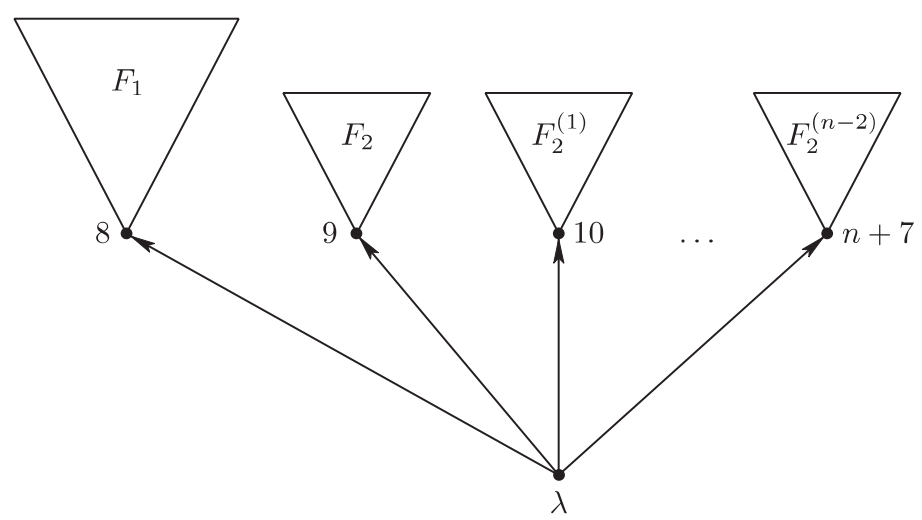

Рис. 14

подшкала $E^{+}$. Иными словами, нужно доказать, что в $E^{+}$нет стрелок, ведущих из $F^{+}$в его дополнение.

В самом деле, допустим противное. Удалив все эти стрелки из $E^{+}$, получим шкалу $E^{\prime}$. Тогда $E^{\prime} \vDash \Gamma$. Действительно, если какое-то условие из $\Gamma$ вида $R_{i_{1}} \circ \cdots \circ R_{i_{s}} \subseteq R_{k}$ нарушается в $E^{\prime}$, то в $E^{\prime}$ для некоторых $x, y$ имеем $x\left(R_{i_{1}} \circ\right.$ $\left.\cdots \circ R_{i_{s}}\right) y$, но при этом неверно $x R_{k} y$. Но так как $E^{+} \vDash \Gamma$, то $x R_{k} y$ в $E^{+}$, и, следовательно, стрелка $(x, y, k)$ была удалена в $E^{\prime}$, т. е. $x \in F^{+}, y \notin F^{+}$. Однако, по построению, в $E^{\prime}$ не может быть цепочки стрелок из $x$ вовне $F^{+}$, а потому неверно $x\left(R_{i_{1}} \circ \cdots \circ R_{i_{s}}\right) y$ - противоречие.

Так как $E^{\prime} \vDash \Gamma$ и $E^{\prime} \subseteq E^{+}$, то из минимальности хорновского замыкания получаем $E^{+}=E^{\prime}$. Поэтому $F^{+}-$коническая подшкала $E^{+}$. Теорема доказана.

Доказанная теорема, по-видимому, распространяется и на другие логики $\boldsymbol{\Lambda}$ из теоремы 4.2, но это требует отдельного исследования. Очевидный пример логики $\boldsymbol{\Lambda}$, для которой утверждение теоремы 5.2 неверно $-\mathbf{K}+\square \perp$. В этом случае единственное $\boldsymbol{\Lambda}$-дерево - одноэлементное, но в его квадрате Сегерберга общезначима формула $p \supset \bigcirc p$, которая не лежит в $\boldsymbol{\Lambda}$ (она опровергается на квадрате шкалы из 2 точек с пустым отношением).

Другой пример - логика S5. В данном случае замыкание дерева - это шкала с универсальным отношением. Логика квадратов Сегерберга таких шкал была аксиоматизирована в [13]. Она содержит формулу $\boxminus p \supset \bigcirc_{1} p$, невыводимую в $\mathbf{S} 5_{\mathrm{Sg}}^{2}$.

Сделаем еще одно дополнение к теореме 5.2.

Лемма 5.3. Пусть $\Lambda$ - логика из теоремы 5.2, $A$ - формула в ее языке, $\boldsymbol{\Lambda}_{\mathrm{Sg}}^{2} \nvdash A$. Тогда $G_{\mathrm{Sg}}^{2},(8,9) \not \models A$ для некоторой $G \in \mathscr{C}_{\boldsymbol{\Lambda}}$.

ДокАЗАТЕЛьство. (Как и в доказательстве теоремы 5.2, мы будем опускать индекс Г.) По лемме $4.10,\left(F^{+}\right)_{\mathrm{Sg}}^{2},(8,9) \not \models A$, где $F$ - коническая подшкала в $T_{\omega, n}$. В доказательстве теоремы 5.2 построена шкала $E^{+} \in \mathscr{C}_{\boldsymbol{\Lambda}}$ такая, что $F^{+}$ коническая подшкала $E^{+}$, и, следовательно, $\left(F^{+}\right)_{\mathrm{Sg}}^{2}-$ коническая подшкала $\left(E^{+}\right)_{\mathrm{Sg}}^{2}$. По лемме о конусах, тогда $\left(E^{+}\right)_{\mathrm{Sg}}^{2},(8,9) \not \models A$. Лемма доказана. 


\section{6. Финитная аппроксимируемость}

В этом разделе мы докажем финитную аппроксимируемость (и, как следствие, разрешимость) логики $\left(\mathbf{K}_{n}\right)_{\mathrm{Sg}}^{2}$. Этот результат, однако, не распространяется на все квадраты логик хорновского типа: даже для самих этих логик финитная аппроксимируемость далеко не всегда известна; существуют также примеры финитно аппроксимируемых логик с неразрешимыми квадратами например, K4, S4 [10].

ОПРЕДЕЛЕНИЕ 6.1. Пусть $F=\left(W, R_{1}, \ldots, R_{n}\right)$ есть $n$-шкала. Путь в $F$ длины $(m-1)$ из $x$ в $y$ - это последовательность точек $x=x_{1}, \ldots, x_{m}=y$ такая, что $x_{i}\left(R_{1} \cup \cdots \cup R_{n}\right) x_{i+1}$ для всех $i$. Глубина $d(x)$ точки $x$ в $F$ - это наибольшая длина пути в $F$ из $x$, если она существует, и $\infty$ в противном случае. Глубина шкаль $F$ (обозначение: $d_{F}$ ) - это наибольшая из глубин ее точек, если она существует, и $\infty$ в противном случае.

Если $F=F \uparrow u-$ конус, то ясно, что $d_{F}=d(u)$, поскольку любой путь из $x \neq u$ можно удлинить, добавив в его начало путь из $u$ в $x$.

Лемма 6.2. Пусть $F=\left(W, R_{1}, \ldots, R_{n}\right)$ есть $n$-икала, $m \geqslant 1$. Рассмотрим n-модальную формулу

$$
\square^{(m)} \perp:=\bigwedge\left\{\square_{\alpha} \perp \mid \alpha \in\{1, \ldots, n\}^{m}\right\} .
$$

Тогда (в любой модели Крипке на $F$ ) для всех $x \in W$

$$
x \vDash \square^{(m)} \perp \Longleftrightarrow d(x) \leqslant m-1 .
$$

ДокАЗАТЕЛЬСТво достаточно очевидно. Применяем индукцию по $m$, используя следующую эквивалентность:

$$
d(x) \leqslant m \Longleftrightarrow \forall y \in\left(R_{1} \cup \cdots \cup R_{n}\right)(x) d(y) \leqslant m-1 .
$$

Лемма доказана.

Зафиксируем теперь логику $\boldsymbol{\Lambda}_{0}=\left(\mathbf{K}_{n}\right)_{\mathrm{Sg}}^{2}$.

ОПРЕДЕЛЕНИЕ 6.3 . Пусть $F=\left(W, X_{1}, \ldots, X_{n}, Y_{1}, \ldots, Y_{n}, f_{0}, f_{1}, f_{2}\right)$ есть $\boldsymbol{\Lambda}_{0}$-шкала. Горизонталъной глубиной $d_{1}(x)$ точки $x$ в $F$ называется глубина $x$ в шкале $F_{1}:=\left(W, X_{1}, \ldots, X_{n}\right)$. Аналогично, вертикальной глубиной $d_{2}(x)$ точки $x$ в $F$ называется глубина $x$ в $F_{2}:=\left(W, Y_{1}, \ldots, Y_{n}\right)$. Горизонтальной (соответственно вертикальной) глубиной $F$ называется глубина $F_{1}$ (соответственно $F_{2}$ ).

Из леммы 6.2 сразу же получаем следующую лемму.

Лемма 6.4. Справедливы следующие эквивалентности:

$$
\begin{aligned}
& x \vDash \Xi^{(m)} \perp \Longleftrightarrow d_{1}(x) \leqslant m-1, \\
& x \vDash \square^{(m)} \perp \Longleftrightarrow d_{2}(x) \leqslant m-1,
\end{aligned}
$$

где

$$
\begin{aligned}
& \boxminus^{(m)} \perp:=\bigwedge\left\{\boxminus_{\alpha} \perp \mid \alpha \in\{1, \ldots, n\}^{m}\right\}, \\
& \square^{(m)} \perp:=\bigwedge\left\{\varpi_{\alpha} \perp \mid \alpha \in\{1, \ldots, n\}^{m}\right\} .
\end{aligned}
$$


Лемма 6.5. В условиях определения 6.3:

(1) $d_{2}\left(f_{0}(x)\right)=d_{1}(x)$,

(2) $d_{1}\left(f_{0}(x)\right)=d_{2}(x)$,

(3) $d_{1}\left(f_{1}(x)\right)=d_{2}\left(f_{1}(x)\right)=d_{2}(x)$,

(4) $d_{1}\left(f_{2}(x)\right)=d_{2}\left(f_{2}(x)\right)=d_{1}(x)$,

(5) $x X_{i} y \Longrightarrow d_{2}(x)=d_{2}(y) \& d_{1}(y) \leqslant d_{1}(x)-1$,

(6) $x Y_{i} y \Longrightarrow d_{1}(x)=d_{1}(y) \& d_{2}(y) \leqslant d_{2}(x)-1$.

Здесь предполагается, что $\infty-1=\infty$ (хотя этот случай нам и не понадобится).

ДоказАтельство. 1) Пусть $d_{1}(x) \geqslant m$. Тогда существует путь $x, x_{1}, \ldots, x_{m}$ в шкале $F_{1}$, и из $(\mathrm{Sg} 5)$ следует, что $f_{0}(x), f_{0}\left(x_{1}\right), \ldots, f_{0}\left(x_{m}\right)$ - путь в $F_{2}$. Отсюда $d_{2}\left(f_{0}(x)\right) \geqslant m$.

Обратно, если $d_{2}\left(f_{0}(x)\right) \geqslant m$, то существует путь $f_{0}(x), y_{1}, \ldots, y_{m}$ в $F_{2}$. Тогда по $(\mathrm{Sg} 5)$ и $(\mathrm{Sg} 1)$ получаем, что $x, f_{0}\left(y_{1}\right), \ldots, f_{0}\left(y_{m}\right)-$ путь в $F_{1}$, и, значит, $d_{1}(x) \geqslant m$.

Таким образом, для любого $m$ имеем $d_{1}(x) \geqslant m \Leftrightarrow d_{2}\left(f_{0}(x)\right) \geqslant m$. Следовательно, $d_{1}(x)=d_{2}\left(f_{0}(x)\right)$.

2) Получается из 1$)$ заменой $x$ на $f_{0}(x)$.

3) Первое равенство следует из 1$)$, так как $f_{0}\left(f_{1}(x)\right)=f_{1}(x)$ по $(\mathrm{Sg} 4)$.

Для проверки второго равенства заметим, что

$$
\boldsymbol{\Lambda}_{0} \vdash \square^{(m)} \perp \equiv \bigcirc_{1} \square^{(m)} \perp .
$$

Это можно доказать и синтаксически, но легче использовать теорему полноты 5.2: достаточно убедиться, что данная формула истинна в $G=F_{\mathrm{Sg}}^{2}$, где $F-$ стандартное $n$-дерево, т. е. что в $F_{\mathrm{Sg}}^{2}$

$$
d_{2}(x)<m \Longleftrightarrow d_{2}\left(\sigma_{1}(x)\right)<m,
$$

или

$$
d_{2}(x) \geqslant m \Longleftrightarrow d_{2}\left(\sigma_{1}(x)\right) \geqslant m .
$$

В самом деле, пусть $d_{2}(\alpha, \beta) \geqslant m$. Тогда в $G$ найдется путь длины $m$ по вертикальным отношениям: $(\alpha, \beta) \sqsubset_{i_{1} 2}\left(\alpha, \beta_{1}\right) \sqsubset_{i_{2} 2} \cdots \sqsubset_{i_{m} 2}\left(\alpha, \beta_{m}\right)$, т. е. $\beta \sqsubset_{i_{1}}$ $\beta_{1} \sqsubset_{i_{2}} \cdots \sqsubset_{i_{m}} \beta_{m}$, и, следовательно, $(\beta, \beta) \sqsubset_{i_{1} 2}\left(\beta, \beta_{1}\right) \sqsubset_{i_{2} 2} \ldots \sqsubset_{i_{m} 2}\left(\beta, \beta_{m}\right)$, откуда $d_{2}(\beta, \beta) \geqslant m$. Аналогично доказывается, что

$$
d_{2}(\beta, \beta) \geqslant m \Longrightarrow d_{2}(\alpha, \beta) \geqslant m \text {. }
$$

4) По $(\mathrm{Sg} 3), f_{2}(x)=f_{1}\left(f_{0}(x)\right)$. Теперь подставим $f_{0}(x)$ вместо $x$ в 3$)$ и используем 2).

5) Достаточно доказать, что

$$
\begin{aligned}
& x X_{i} y \& d_{1}(y) \geqslant m \Longrightarrow d_{1}(x) \geqslant m+1, \\
& x X_{i} y \Longrightarrow\left(d_{2}(x) \geqslant m \Leftrightarrow d_{2}(y) \geqslant m\right) .
\end{aligned}
$$

Первое утверждение очевидно: если $x X_{i} y$ и в $F_{1}$ из $y$ выходит путь длины $m$, то, добавив в его начало $x$, получим путь из $x$ длины $m+1$. 
Второе утверждение следует из

$$
\boldsymbol{\Lambda}_{0} \vdash \square^{(m)} \perp \supset \boxminus_{i} \square^{(m)} \perp, \diamond_{i} \square^{(m)} \perp \supset \square^{(m)} \perp .
$$

Обе эти формулы легко проверяются на квадратах деревьев, но их можно вывести непосредственно: первую - используя аксиому коммутативности и $\perp \supset \boxminus_{i} \perp$, вторую - с помощью аксиомы Чёрча-Россера и $\diamond_{i} \perp \supset \perp$.

$6)$ следует из 5$)$, если заменить $x$ на $f_{0}(x)$ и использовать 1$)$ и 2 ).

Лемма доказана.

ОПРЕДЕЛЕНиЕ 6.6. Определим длину пары кортежей натуральных чисел:

$$
|(\alpha, \beta)|:=\max (|\alpha|,|\beta|) .
$$

ОПРЕДЕлЕниЕ 6.7. Степенъ $d(A)$ модальной формулы $A$ в языке $\left(\mathbf{K}_{n}\right)_{\mathrm{Sg}}^{2}$ определяется по индукции:

$$
\begin{aligned}
d\left(p_{k}\right) & =d(\perp)=0 ; & d(A \supset B) & =\max (d(A), d(B)) ; \\
d(\bigcirc A) & =d\left(\bigcirc_{i} A\right)=d(A) ; & d\left(\square_{j} A\right) & =d\left(\boxminus_{j} A\right)=d(A)+1 .
\end{aligned}
$$

ОПРЕДЕЛЕНИЕ 6.8. Пусть $F \subseteq T_{\omega, n}-$ стандартное дерево. Из него получается усеченное дерево высоты $m: F^{(m)}:=F\lceil\{\alpha|| \alpha \mid \leqslant m\}$.

При фиксированном $m$ мы будем обозначать $F^{(m)}$ через $F^{-}$.

Лемма 6.9 (лемма об усечении). Пусть $F \subseteq T_{\omega, n}-$ стандартное дерево, $F^{-}=F^{(m)}, M-$ модель Крипке на $F_{\mathrm{Sg}}^{2}, M^{-}-$ее ограничение на $\left(F^{-}\right)_{\mathrm{Sg}}^{2}$. Тогда для любой формулы $A$ в языке $\left(\mathbf{K}_{n}\right)_{\mathrm{Sg}}^{2}$, для любого $x \in\left(F^{-}\right)^{2}$, если $d(A)+|x| \leqslant m$, mo

$$
M, x \vDash A \Longleftrightarrow M^{-}, x \vDash A .
$$

ДокАзАТЕльство. Индукцией по $|A|$ доказываем утверждение для всех $x$. Если $A \in \mathrm{PV}$, утверждение верно по определению $M^{-}$.

Случаи $A=\perp$ и $A=B \supset C$ очевидны.

Пусть $A=\bigcirc B$ и $d(A)+|x| \leqslant m$. Тогда

$$
M, x \vDash A \Longleftrightarrow M, \sigma(x) \vDash B, \quad M^{-}, x \vDash A \Longleftrightarrow M^{-}, \sigma(x) \vDash B .
$$

Но $|x|=|\sigma(x)|, d(A)=d(B)$, поэтому $d(B)+|\sigma(x)| \leqslant m$ и можно применить предположение индукции для $B$ :

$$
M, \sigma(x) \vDash B \Longleftrightarrow M^{-}, \sigma(x) \vDash B .
$$

Отсюда следует утверждение для $A$.

Аналогично, пусть $A=\bigcirc_{j} B(j=1,2)$ и $d(A)+|x| \leqslant m$. Тогда

$$
M, x \vDash A \Longleftrightarrow M, \sigma_{j}(x) \vDash B, \quad M^{-}, x \vDash A \Longleftrightarrow M^{-}, \sigma_{j}(x) \vDash B .
$$

Заметим, что $|(\alpha, \alpha)|,|(\beta, \beta)| \leqslant|(\alpha, \beta)|$, т. е. $\left|\sigma_{j}(x)\right| \leqslant|x|$. Кроме того, $d(A)=$ $d(B)$, поэтому $d(B)+\left|\sigma_{j}(x)\right| \leqslant d(A)+|x| \leqslant m$ и опять можно применить предположение индукции для $B$.

Пусть $A=\boxminus_{i} B$ и $d(A)+|x| \leqslant m$. Тогда

$$
\begin{gathered}
M, x \vDash A \Longleftrightarrow \forall y \in M\left(x \sqsubset_{i 1} y \Rightarrow M, y \vDash B\right), \\
M^{-}, x \vDash A \Longleftrightarrow \forall y \in M\left(x \sqsubset_{i 1} y \Rightarrow M^{-}, y \vDash B\right),
\end{gathered}
$$


так как в $M$ и $M^{-}$из $x$ достижимы одни и те же точки (если $|x|<m$, как в данном случае).

Заметим, что $x \sqsubset_{i 1} y \Rightarrow|y| \leqslant|x|+1$. Поэтому $d(B)+|y| \leqslant d(B)+1+|x|=$ $d(A)+|x| \leqslant m$ и снова можно применить предположение индукции для $B$.

Случай $A=\varpi_{i} B$ разбирается аналогично.

Лемма доказана.

ЛЕмма 6.10. $\boldsymbol{\Lambda}_{0}=\bigcap_{m}\left(\boldsymbol{\Lambda}_{0}+\boxminus^{(m)} \perp\right)$.

ДоказАтельство. Очевидно, достаточно показать, что если $\boldsymbol{\Lambda}_{0} \nvdash A$, то $\boldsymbol{\Lambda}_{0}+\boxminus^{(m)} \perp \nvdash A$ для некоторого $m$.

Итак, пусть $\boldsymbol{\Lambda}_{0} \nvdash A$. Тогда, по лемме $5.3, G_{\mathrm{Sg}}^{2},(8,9) \not \models A$ для некоторого стандартного дерева $G$.

Пусть $m=d(A)+1, G^{-}=G^{(m)}$ (см. определение 6.8). Так как $d(A)+|(8,9)|=$ $m$, то, по лемме $6.9,\left(G^{-}\right)_{\mathrm{Sg}}^{2},(8,9) \not \models A$. Теперь заметим, что $\left(G^{-}\right)_{\mathrm{Sg}}^{2} \vDash \boxminus^{(m+1)} \perp$.

Действительно, $(\alpha, \beta) \sqsubset_{i 1}(\gamma, \beta) \Rightarrow|\gamma|=|\alpha|+1$. Поэтому, если в шкале $\left(G^{-}\right)^{2}$ с отношениями $\sqsubset_{11}, \ldots, \sqsubset_{n 1}$ есть путь $\left(\alpha_{1}, \beta\right), \ldots,\left(\alpha_{k}, \beta\right)$, то его длина равна $k-1=\left|\alpha_{k}\right|-\left|\alpha_{1}\right| \leqslant m$. Таким образом, глубина точек в этой шкале не превосходит $m$, откуда, по лемме 6.2 , и следует наше утверждение.

Следовательно, $\boldsymbol{\Lambda}_{0}+\boxminus^{(m+1)} \perp \nvdash A$.

Лемма доказана.

Наша следующая цель - доказательство локальной табличности логик $\boldsymbol{\Lambda}_{0}+$ $\boxminus^{(m)} \perp$. Для этого мы исследуем ограниченные канонические модели.

ОПРедЕЛЕНИЕ 6.11. Точки $x, y$ модели Крипке $M$ называются подобными (обозначение: $x \asymp_{M} y$ ), если существует бисимуляция $E: M, x \asymp M, y$. Точки $x, y$ называются локально изоморфными (обозначение: $x \cong_{M} y$ ), если существует изоморфизм конусов $f: M \uparrow x \longrightarrow M \uparrow y$, переводящий $x$ в $y$.

Очевидно, что локально изоморфные точки подобны, так как изоморфизм конусов является бисимуляцией.

ЛЕмма 6.12 (лемма о жесткости). В ограниченной канонической модели произвольной модальной логики подобные точки совпадают.

ДокАЗАТЕЛьство. Пусть $L-n$-модальная логика, $M-$ ее ограниченная каноническая модель, $x \asymp_{M} y$. По лемме о бисимуляции 2.15 , в $x$ и $y$ истинны одни и те же $n$-модальные формулы. Следовательно, $x=y$ - по теореме 2.6 (i). Лемма доказана.

Будем теперь рассматривать ограниченную каноническую модель с $m$ переменными произвольной $(2 n+3)$-модальной логики $\boldsymbol{\Lambda}_{1} \supseteq \boldsymbol{\Lambda}_{0}$. Как отмечалось в доказательстве леммы 4.3 , логика $\boldsymbol{\Lambda}_{0}=\left[\mathbf{K}_{n}, \mathbf{K}_{n}\right]^{\circledast}$ является d-упорной. Из дескриптивности $\Phi_{\boldsymbol{\Lambda}_{1}\lceil m}$ тогда следует, что $F_{\boldsymbol{\Lambda}_{1}\lceil m} \vDash \boldsymbol{\Lambda}_{0}$. Этим мы будем пользоваться. Обозначим

$$
M:=M_{\Lambda_{1}\lceil m}, \quad F:=F_{\Lambda_{1}\lceil m} .
$$

Отношения и функции в $F$ будем обозначать так же, как в лемме 3.3 , а именно $X_{1}, \ldots, X_{n}, Y_{1}, \ldots, Y_{n}, f_{0}, f_{1}, f_{2}$. 
Точки $x, y$ из $M$ называются 0-эквивалентными (обозначение: $x \equiv_{0} y$ ), если

$$
\forall j \leqslant m\left(M, x \vDash p_{j} \Leftrightarrow M, x \vDash p_{j}\right) .
$$

Класс 0 -эквивалентности $\equiv_{0}(x)$ будем обозначать через $[x]_{0}$.

Лемма 6.13. Пусть $x, y-$ точки модели $M$ такие, что

$$
\begin{gathered}
x \equiv_{0} y \& f_{0}(x) \equiv_{0} f_{0}(y) \& f_{1}(x)=f_{1}(y) \& f_{2}(x)=f_{2}(y) \\
\& \forall i \leqslant n\left(X_{i}(x)=X_{i}(y) \& Y_{i}(x)=Y_{i}(y)\right) .
\end{gathered}
$$

Тогда $x=y$.

ДокАзАтельство. Рассмотрим следующее отношение на $M$ :

$$
E:=\left\{(x, y),\left(f_{0}(x), f_{0}(y)\right)\right\} \cup\{(z, z) \mid z \in M \uparrow x\} .
$$

Докажем, что $E: M, x \asymp M, y$.

(1) $u E v \Rightarrow f_{0}(u) E f_{0}(v)$.

Если $u=v$, то доказывать нечего. Если $u=x, v=y$, то $f_{0}(u) E f_{0}(v)$ по определению. Если же $u=f_{0}(x), v=f_{0}(y)$, то $f_{0}(u)=x, f_{0}(v)=y($ по (Sg1)) и снова $f_{0}(u) E f_{0}(v)$.

(2) $u E v \Rightarrow f_{1}(u) E f_{1}(v)$.

При $u=v$ все тривиально. Если $u=x, v=y$, то $f_{1}(u)=f_{1}(v)$ по условию леммы и $f_{1}(u) E f_{1}(v)$ - по определению $E$.

Если $u=f_{0}(x), v=f_{0}(y)$, то $f_{1}(u)=f_{1} f_{0}(x)=f_{2}(x)$; аналогично, $f_{1}(v)=$ $f_{2}(y)$. По условию леммы и определению $E$, опять получаем $f_{1}(u) E f_{1}(v)$.

(3) $u E v \Rightarrow f_{2}(u) E f_{2}(v)$.

Сразу следует из (1) и (2).

(4) $u E v X_{i} w \Rightarrow \exists t\left(u X_{i} t E w\right)$. Действительно, можно взять $t=w$. При $u=v$ это очевидно. Если $u=x, v=y$, то используем условие $X_{i}(x)=X_{i}(y)$. Если же $u=f_{0}(x), v=f_{0}(y)$, то используем условие $Y_{i}(x)=Y_{i}(y)$, которое можно переписать как $X_{i}\left(f_{0}(x)\right)=X_{i}\left(f_{0}(y)\right)$, заметив, что из $(\mathrm{Sg} 5)$, (Sg1) следует равенство $X_{i}\left(f_{0}(z)\right)=f_{0}\left(Y_{i}(z)\right)$.

(5) $u E v \& u X_{i} w \Rightarrow \exists t\left(v X_{i} t \& w E t\right)$.

И здесь можно взять $t=w$. Доказательство аналогично (4), с использованием условий $X_{i}(x)=X_{i}(y), Y_{i}(x)=Y_{i}(y)$.

(6) $u E v Y_{i} w \Rightarrow \exists t\left(u Y_{i} t E w\right)$.

Следует из (Sg5), (Sg1), (5) и (1): применив $f_{0}$, превращаем (6) в (5).

(7) $u E v \& u Y_{i} w \Rightarrow \exists t\left(v Y_{i} t \& w E t\right)$.

Аналогично - следует из (4).

Свойства (1)-(7) вместе с условием $x \equiv_{0} y \& f_{0}(x) \equiv_{0} f_{0}(y)$ показывают, что $E: M, x \asymp M, y$. Теперь можно применить лемму о жесткости 6.12. Лемма доказана.

ЛЕмма 6.14. Для любого конечного $r$ множество

$$
S_{r}:=\left\{x \in M \mid \max \left(d_{1}(x), d_{2}(x)\right)=r\right\}
$$

конечно. 
ДокАзАТЕльство. Для удобства сначала введем некоторые термины и обозначения. Множество $S_{r}$ называется $r$-м ярусом в $M$. Оно разбивается на секторы - горизонтальные

$$
S_{k r}:=\left\{x \in M \mid d_{1}(x)=k, d_{2}(x)=r\right\}
$$

и вертикальные

$$
S_{r k}:=\left\{x \in M \mid d_{1}(x)=r, d_{2}(x)=k\right\},
$$

где $0 \leqslant k \leqslant r$. В свою очередь, секторы разбиваются на ячейки. Каждой диагональной точке $a$ яруса $r$ (т. е. точке $a \in S_{r r}$ такой, что $f_{1}(a)=a$ ) отвечают горизонтальные ячейки

$$
S_{k r}(a):=\left\{x \in S_{k r} \mid f_{1}(x)=a\right\}
$$

и вертикальные ячейки

$$
S_{r k}(a):=\left\{x \in S_{r k} \mid f_{2}(x)=a\right\}
$$

$($ где $0 \leqslant k \leqslant r)$. Ячейка $S_{r r}(a)$ - горизонтальная и вертикальная одновременно.

Перепишем лемму 6.5 в этих обозначениях:

(0.1) $f_{0}\left[S_{k l}\right]=S_{l k}$

(0.2) $f_{1}\left[S_{k l}\right] \subseteq S_{l l}$,

(0.3) $f_{2}\left[S_{k l}\right] \subseteq S_{k k}$,

(0.4) $X_{i}\left[S_{k l}\right] \subseteq \bigcup_{j<k} S_{j l}$,

(0.5) $Y_{i}\left[S_{k l}\right] \subseteq \bigcup_{j<l} S_{k j}$.

Отсюда получается, что $S_{0} \cup \cdots \cup S_{r}$ замкнуто относительно $f_{0}, f_{1}, f_{2}, X_{i}, Y_{i}$, и значит,

(1) $S_{0} \cup \cdots \cup S_{r}-$ коническое подмножество $M$.

(2) $S_{0}$ содержит конечное число диагональных точек.

Действительно, для любой диагональной точки $x$ имеем $x=f_{0}(x)=f_{1}(x)$, $X_{i}(x)=Y_{i}(x)=\varnothing$. Значит, конус $M \uparrow x$ состоит только из $x$, и поэтому (для диагональных точек $\left.S_{0}\right)$

$$
M \uparrow x \cong M \uparrow y \Longleftrightarrow x \equiv_{0} y
$$

откуда

$$
x \equiv_{0} y \Longleftrightarrow x=y
$$

- по лемме 6.12. Но в $M$ имеется конечное число классов по $\equiv_{0}$ (не более $2^{m}$ ); следовательно, $S_{0}$ содержит не более $2^{m}$ диагональных точек (на самом деле, можно показать, что их в точности $\left.2^{m}\right)$.

(3) $S_{0}$ конечно.

Из $(2)$ следует, что функции $f_{1}$ и $f_{2}$ принимают конечное число значений на $S_{0}$ (напомним, что точка $f_{2}(x)=f_{1}\left(f_{0}(x)\right)$ - диагональная). Для $x \in S_{0}$ всегда $X_{i}(x)=Y_{i}(x)=\varnothing$. Тогда по лемме 6.13 точка $x$ однозначно определяется четверкой $\left([x]_{0},\left[f_{0}(x)\right]_{0}, f_{1}(x), f_{2}(x)\right)$. Число таких четверок конечно - не более $2^{4 m}$.

(4) Предположим, что $r>0$ и все ярусы $S_{k}$ при $k<r$ конечны. Покажем, что ярус $S_{r}$ конечен. Доказательство разбивается на несколько утверждений. 
(4.1) При $0 \leqslant k \leqslant r$

$$
f_{0}\left[S_{k r}(a)\right]=S_{r k}(a), \quad f_{0}\left[S_{r k}(a)\right]=S_{k r}(a) .
$$

Действительно, если $x \in S_{k r}$ и $f_{1}(x)=a$, то $f_{0}(x) \in S_{r k}, f_{2} f_{0}(x)=f_{1}(x)=a-$ по (0.1), (Sg3), (Sg1). Таким образом,

$$
f_{0}\left[S_{k r}(a)\right] \subseteq S_{r k}(a) .
$$

Отсюда, по (Sg1),

$$
S_{k r}(a)=f_{0} f_{0}\left[S_{k r}(a)\right] \subseteq f_{0}\left[S_{r k}(a)\right] .
$$

Аналогично получаем

$$
f_{0}\left[S_{r k}(a)\right] \subseteq S_{k r}(a), \quad S_{r k}(a) \subseteq f_{0}\left[S_{k r}(a)\right] .
$$

(4.2) Все ячейки сектора $S_{0 r}$ конечны и в совокупности ограничены по мощности.

В самом деле, $X_{i}(x)=\varnothing$ для $x \in S_{0 r}$ и, по лемме 6.5 ,

$$
Y_{i}(x) \subseteq \bigcup_{j<r} S_{0 j} \subseteq S_{0} \cup \cdots \cup S_{r-1}, \quad f_{2}(x) \in S_{0} .
$$

По лемме 6.13 , внутри одной ячейки (т. е. при фиксированном $f_{1}(x)$ ) точка $x$ однозначно определяется четверкой $\left([x]_{0},\left[f_{0}(x)\right]_{0}, f_{2}(x), Y_{i}(x)\right)$.

Число таких четверок конечно - не более $2^{2 m}\left|S_{0}\right| \cdot 2^{\left|S_{0} \cup \cdots \cup S_{r-1}\right|}$, что и ограничивает мощность ячейки.

(4.3) При $k \leqslant r$ все ячейки сектора $S_{k r}$ конечны и в совокупности ограничены по мощности.

Доказательство - индукцией по $k$. Базис доказан в (4.2).

Пусть $0<k \leqslant r$ и утверждение верно для $k-1$. Доказываем его для $k$. По свойствам (0.4), (0.5), (0.3), для $x \in S_{k r}$ имеем:

$$
X_{i}(x) \subseteq \bigcup_{j<k} S_{j r}, \quad Y_{i}(x) \subseteq \bigcup_{j<r} S_{k j} \subseteq S_{0} \cup \cdots \cup S_{r-1}, \quad f_{2}(x) \in S_{k k} \subseteq S_{k} .
$$

По лемме 6.13 , внутри одной ячейки точка $x$ однозначно определяется пятер-

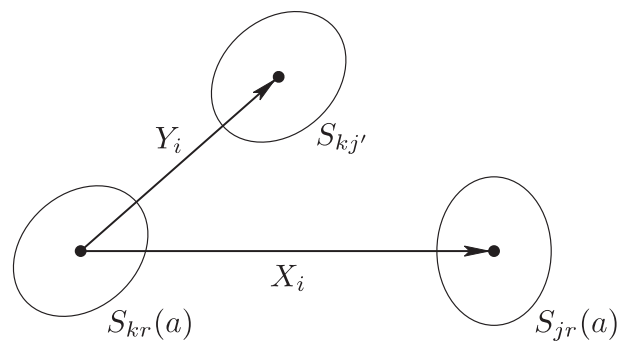

Рис. 15

кой $\left([x]_{0},\left[f_{0}(x)\right]_{0}, f_{2}(x), X_{i}(x), Y_{i}(x)\right)$. Число таких пятерок конечно - не более $2^{2 m}\left|S_{k}\right| \cdot 2^{\left|S_{0 r} \cup \cdots \cup S_{(k-1) r}\right|} \cdot 2^{\left|S_{0} \cup \cdots \cup S_{r-1}\right|}$. Отсюда следует утверждение для $k$. 
(4.4) Все вертикальные ячейки яруса $S_{r}$ конечны и в совокупности ограничены по мощности.

Это следует из (4.3) и (4.1).

(4.5) Для диагональных точек $a \in S_{r}$ конусы $M \uparrow a$ конечны и в совокупности ограничены по мощности.

Сначала заметим, что (как множество) $M \uparrow a$ содержится в

$$
P:=\{a\} \cup S_{0} \cup \cdots \cup S_{r-1} \cup \bigcup_{k=0}^{r-1} S_{k r}(a) \cup \bigcup_{k=0}^{r-1} S_{r k}(a) .
$$

Для этого покажем, что множество $P$ - коническое. Достаточно проверить его замкнутость относительно $f_{0}, f_{1}, X_{i}$.

Очевидно, что $f_{0}(a)=a$, так как $a$ диагональна; из (1) получаем:

$$
f_{0}\left[S_{0} \cup \cdots \cup S_{r-1}\right] \subseteq S_{0} \cup \cdots \cup S_{r-1} .
$$

Кроме того, по (4.1), $f_{0}\left[S_{k r}(a)\right]=S_{r k}(a), f_{0}\left[S_{r k}(a)\right]=S_{k r}(a)$. Таким образом, $f_{0}[P] \subseteq P$.

Далее, $f_{1}(a)=a$, так как $a$ диагональна;

$$
f_{1}\left[S_{0} \cup \cdots \cup S_{r-1}\right] \subseteq S_{0} \cup \cdots \cup S_{r-1}
$$

- по (1). Если $x \in S_{r k}, k<r$, и $f_{2}(x)=a$, то $f_{1}(x) \in S_{k k} \subseteq S_{k} \subseteq P$ - по (0.2) и определению $P$. Если же $f_{1}(x)=a$, то очевидно, что $f_{1}(x) \in P$. Итак, $f_{1}[P] \subseteq P$.

Пусть теперь $x \in P, x X_{i} y$. Тогда $y \in P$. Действительно, при $x \in S_{0} \cup \cdots \cup S_{r-1}$ имеем $y \in S_{0} \cup \cdots \cup S_{r-1}-$ по (1). Если $x=a$, то $x \in S_{r r}$, поэтому $y \in S_{(r-1) r} \cup$ $\cdots \cup S_{0 r}-$ по (0.4); кроме того, $f_{1}(y)=f_{1}(x)=a-$ по $(\mathrm{Sg} 7)$. Аналогично, если $x \in S_{k r}, k<r$, то $y \in S_{(k-1) r} \cup \cdots \cup S_{0 r}-$ по $(0.4)$; кроме того, $f_{1}(y)=f_{1}(x)=a-$ по (Sg7). Если $x \in S_{r k}, k<r$, то, по (0.4), $y \in S_{(r-1) k} \cup \cdots \cup S_{0 k} \subseteq S_{r-1} \cup \cdots \cup S_{k}$. Значит, $X_{i}(P) \subseteq P$.

Таким образом, $P$ - коническое множество, содержащее $a$; тогда оно содержит и весь конус $M \uparrow a$. Но $P$ получается из конечного множества $\{a\} \cup S_{0} \cup$ $\cdots \cup S_{r-1}$ добавлением $2 r$ ячеек ограниченной мощности - ввиду (4.3), (4.4). Следовательно, $|M \uparrow a| \leqslant N$ для некоторого $N$, не зависящего от $a$.

(4.6) $S_{r}$ содержит конечное число диагональных точек.

Ввиду аксиом (Sg1), (Sg3), (Sg5), $\boldsymbol{\Lambda}_{1}$-шкала с данным множеством миров однозначно определяется функциями $f_{0}, f_{1}$ и отношениями $X_{1}, \ldots, X_{n}$. Поэтому для задания $\boldsymbol{\Lambda}_{1}$-шкалы мощности $s$ с точностью до изоморфизма достаточно определить эти отношения и функции на фиксированном множестве из $s$ элементов. Следовательно, число классов изоморфизма $\boldsymbol{\Lambda}_{1}$-шкал мощности $s$ не превосходит $\left(s^{s}\right)^{2}\left(2^{s^{2}}\right)^{n}$.

Оценку каждой пропозициональной переменной на такой шкале можно выбрать $2^{s}$ способами, и потому число неизоморфных моделей Крипке мощности $s$ с фиксированными значениями переменных $p_{i}$ при $i>m$ (как в канонической модели $\left.M_{L_{1}\lceil m}\right)$ не превосходит $s^{2 s} \cdot 2^{n s^{2}} \cdot 2^{m s}$. А число таких моделей мощности $\leqslant N$ не превосходит

$$
q:=\sum_{s=1}^{N}\left(s^{2 s} \cdot 2^{n s^{2}+m s}\right)
$$


Очевидно, что точку внутри данной модели мощности $s$ можно выбрать $s$ способами. С учетом (4.5), получаем отсюда, что число локально неизоморфных диагональных точек в $S_{r}$ не превосходит $q N$. Тогда и число этих точек не превосходит $q N$ - благодаря жесткости.

(4.7) Все секторы яруса $S_{r}$ конечны.

Действительно, из (4.6), (4.3), (4.4) следует, что каждый сектор разбивается на конечное число конечных ячеек. Лемма 6.14 доказана.

ЛЕмма 6.15. Каждая логика $\boldsymbol{\Lambda}_{0}+\boxminus^{(r)} \perp$ локалъно таблична.

ДокАЗАтЕльство. Пусть $\boldsymbol{\Lambda}_{1}:=\boldsymbol{\Lambda}_{0}+\boxminus^{(r)} \perp$. Формула $\boxminus^{(r)} \perp$ замкнута, и потому d-упорна. Тогда $F_{\boldsymbol{\Lambda}_{1}\lceil m} \vDash \boxminus^{(r)} \perp$, и, значит, по лемме $6.4, d_{1}(x)<r$ для всех $x \in F_{\Lambda_{1}\lceil m}$.

Заметим, что $\left(\boxminus^{(r)} \perp\right)^{\circ}=\square^{(r)} \perp$, и потому, по лемме $3.5(2)$, имеем $\boldsymbol{\Lambda}_{1} \vdash \square^{(r)} \perp$. Тогда $F_{\Lambda_{1}\lceil m} \vDash \square^{(r)} \perp$ и, по лемме 6.4 , получаем, что $d_{2}(x)<r$ для всех $x \in$ $F_{\boldsymbol{\Lambda}_{1}\lceil m}$. Таким образом, $F_{\boldsymbol{\Lambda}_{1}\lceil m}$ содержится в объединении ярусов $S_{0} \cup \cdots \cup S_{r-1}$, и потому она конечна - по лемме 6.14 .

ТеОрема 6.16. Логика $\left(\mathbf{K}_{n}\right)_{\mathrm{Sg}}^{2}$ финитно аппроксимируема.

ДокАЗАТЕЛЬство. Согласно леммам 6.10 и $6.15,\left(\mathbf{K}_{n}\right)_{\mathrm{Sg}}^{2}-$ пересечение локально табличных логик. Теперь можно применить леммы 2.18 и 2.3 .

Из конечной аксиоматизируемости $\left(\mathbf{K}_{n}\right)_{\mathrm{Sg}}^{2}$ и теоремы 6.16 получаем следующее утверждение.

СЛЕДСТВИЕ 6.17. Логика $\left(\mathbf{K}_{n}\right)_{\mathrm{Sg}}^{2}$ разрешима.

\section{7. Финитная аппроксимируемость квадратами}

В этом разделе мы усилим результат о финитной аппроксимируемости, доказав, что логика $\left(\mathbf{K}_{n}\right)_{\mathrm{Sg}}^{2}$ полна относительно конечных шкал, которые сами являются квадратами Сегерберга. Аналог этого свойства для обычных произведений - финитная аппроксимируемость произведениями (product fmp) - был доказан для ряда логик в [6].

ОПРЕДЕЛЕНИЕ 7.1. Пусть $\boldsymbol{\Lambda}-n$-модальная логика. Мы говорим, что ее квадрат Сегерберга $\boldsymbol{\Lambda}_{\mathrm{Sg}}^{2}$ финитно аппроксимируется квадратами, если

$$
\boldsymbol{\Lambda}_{\mathrm{Sg}}^{2}=\mathbf{L}\left(\left\{F_{\mathrm{Sg}}^{2} \mid F \vDash \boldsymbol{\Lambda}, F \text { конечна }\right\}\right) .
$$

Основным результатом этого раздела является следующая теорема.

ТЕОРема 7.2. $\left(\mathbf{K}_{n}\right)_{\text {Sg }}^{2}$ финитно аппроксимируется квадратами.

Перед началом доказательства сделаем небольшое уточнение к теореме 6.16. Будем снова обозначать $\left(\mathbf{K}_{n}\right)_{\mathrm{Sg}}^{2}$ через $\boldsymbol{\Lambda}_{0}$.

ОПРЕДЕЛЕНИЕ 7.3. $\boldsymbol{\Lambda}_{0}$-шкала $G$ называется главным конусом, если $G=$ $G \uparrow u$ для некоторой диагональной точки $u$. 
ПРЕДЛОЖЕНИЕ 7.4. Справедливо равенство

$$
\begin{aligned}
\mathbf{\Lambda}_{0}=\mathbf{L}\left(\left\{G \mid G \vDash \boldsymbol{\Lambda}_{0}, G-\right.\right. & \text { конечный главный конус } \\
& \text { конечной горизонтальной глубины }\}) .
\end{aligned}
$$

Доказательство. Пусть $\boldsymbol{\Lambda}_{0} \nvdash A, m=d(A)+1, A \in \mathscr{L}_{2 n+3}\lceil k$. Из доказательства леммы 6.10 видим, что $\left(G^{-}\right)_{\mathrm{Sg}}^{2},(8,9) \not \models A$ для некоторого стандартного дерева $G^{-}$глубины $\leqslant m$. При этом $\left(G^{-}\right)_{\mathrm{Sg}}^{2} \vDash \boxminus^{(m+1)} \perp$ и $\lambda \sqsubset_{i} 8, \lambda \sqsubset_{j} 9$ для некоторых $i, j$.

Рассмотрим теперь множество формул

$$
\Theta:=\left\{\diamond_{i} \diamond_{j} \neg A\right\} \cup\left\{\bigcirc_{1} B \equiv B \mid B \in \mathscr{L}_{2 n+3}\lceil k\} .\right.
$$

По построению, для некоторой модели $M$ на $\left(G^{-}\right)_{\operatorname{Sg}}^{2}$ имеем $M,(\lambda, \lambda) \vDash \Theta$. Следовательно, $\Theta$ непротиворечиво в логике $\boldsymbol{\Lambda}_{1}:=\boldsymbol{\Lambda}_{0}+\boxminus^{(m+1)} \perp$. Кроме того, $\Theta \subseteq \mathscr{L}_{2 n+3}\lceil k$, поэтому, по свойству ограниченной канонической модели, для некоторого $u$ имеем $M_{\boldsymbol{\Lambda}_{1}\lceil k}, u \vDash \Theta$. По лемме о конусах, тогда $M_{\boldsymbol{\Lambda}_{1}\lceil k} \uparrow u$, $u \vDash \diamond_{i} \diamond_{j} \neg A$, и значит, $F_{\boldsymbol{\Lambda}_{1}\lceil k} \uparrow u \not \models A$. При этом $F_{\boldsymbol{\Lambda}_{1}\lceil k}-$ конечная шкала конечной горизонтальной глубины (см. доказательство леммы 6.15).

Из определения канонической модели и аксиом SL для $\bigcirc_{1}$ в модели $M_{\boldsymbol{\Lambda}_{1}\lceil k}$ имеем:

$$
f_{1}(u)=\left\{B \mid \bigcirc_{1} B \in u\right\} .
$$

Отсюда по построению $\Theta$ получаем $f_{1}(u)=u$, т. е. $u$ - диагональная точка, и конус $F_{\Lambda_{1}\lceil k} \uparrow u-$ главный. Предложение доказано.

Благодаря доказанному предложению и лемме о р-морфизме, для доказательства теоремы 7.2 достаточно представить любой конечный главный $\boldsymbol{\Lambda}_{0}$-конус конечной горизонтальной глубины как р-морфный образ конечного квадрата Сегерберга. Для этого мы снова применим игру - с другими правилами, чем для игры $\operatorname{RG}(G)$ из раздела 4.

Начальная позиция строится на подготовительном этапе игры. Его можно организовать как отдельную игру, но мы воспользуемся конструкцией развертки, которую сейчас напомним.

ОПРЕДЕЛЕНИЕ 7.5. Пусть $k>0$ - натуральное число. р-морфизм шкал $g:\left(W, R_{1}, \ldots, R_{m}\right) \rightarrow\left(W^{\prime}, R_{1}^{\prime}, \ldots, R_{m}^{\prime}\right)$ называется $k$-кратным, если выполняется $k$-кратное свойство поднятия в любой точке $x \in W$ :

$$
g(x) R_{i}^{\prime} y \Longrightarrow\left|\left\{z \mid x R_{i} z \& g(z)=y\right\}\right| \geqslant k .
$$

Лемма 7.6. Пусть $F=\left(W, R_{1}, \ldots, R_{n}\right)$ - конечный конус конечной глубины $r$, и пусть $k>0$. Тогда $F-k$-кратный р-морбный образ конечного дерева глубины $r$.

ДокАЗАТЕЛЬство. Построение можно провести в два шага, как описано в [5]. Пусть $F=F \uparrow u$. Построим сначала утолщение шкалы $F$ :

$$
F_{k}:=\left(V, R_{1}^{\prime}, \ldots, R_{n}^{\prime}\right)
$$

где

$$
V:=\{u\} \times 1 \cup(W-\{u\}) \times k,
$$


при этом $1=\{0\}, k=\{0,1, \ldots, k-1\}$ - как обычно в теории множеств;

$$
(a, i) R_{j}^{\prime}(b, l) \Leftrightarrow a R_{j} b .
$$

Таким образом, в $F_{k}$ имеется $k$ копий каждого некорневого мира из $F$ и одна копия корня; копии связаны теми же отношениями, что их оригиналы. Легко проверить, что проекция $\gamma:(a, i) \mapsto a$ задает $k$-кратный р-морфизм $F_{k} \rightarrow F$. При этом важно, что в $F$ нет стрелок, ведущих в корень $u$ - иначе бы в $F$ имелся цикл, что невозможно в шкале конечной глубины.

Шкала $F_{k}$ - конечный конус с корнем $(u, 1)$; глубина его равна $r$, так как $d(u, 1)=d(u)$.

На следующем шаге строится развертка $F_{k}^{\sharp}$ шкалы $F_{k}$. Напомним ее определение.

Обозначим $v_{0}:=(u, 1)$. Тогда $F_{k}^{\#}:=\left(V^{\#}, R_{1}^{\#}, \ldots, R_{n}^{\#}\right)$, где

$$
\begin{gathered}
V^{\#}:=\left\{\left(v_{0}, j_{0}, v_{1}, \ldots, j_{m-1}, v_{m}\right) \mid m>0 \& \forall i<m v_{i} R_{j_{i}}^{\prime} v_{i+1}\right\} \cup\left\{\left(v_{0}\right)\right\}, \\
\left(v_{0}, \ldots, v_{m}\right) R_{j}^{\#} x \Longleftrightarrow \exists w x=\left(v_{0}, \ldots, v_{m}, j, w\right) .
\end{gathered}
$$

Таким образом, развертка состоит из размеченных путей в $F_{k}$ с началом в корне $v_{0}$. Нетривиальный размеченный путь можно представлять себе как последовательность стрелок. Отношение достижимости $R_{j}^{\#}$ связывает размеченный путь с путями, полученными из него добавлением одной $j$-стрелки в конце.

$F_{k}^{\sharp}$ - конечное дерево с корнем $v_{0}$ (как конус, в котором у каждой некорневой точки есть единственный предшественник). Глубина его, очевидно, равна $r$.

Отображение $\pi:\left(v_{0}, \ldots, v_{m}\right) \mapsto v_{m}$ является р-морфизмом $F_{k}^{\sharp} \rightarrow F_{k}$; поэтому композиция $\gamma \pi: F_{k}^{\sharp} \rightarrow F$ есть искомый $k$-кратный р-морфизм. Лемма доказана.

Лемма 7.7 (основная лемма). Пусть $G$ - конечный главный $\boldsymbol{\Lambda}_{0}$-конус конечной горизонтальной глубины $r$. Тогда для некоторого конечного $n$-дерева $F$ глубины $r$ существует р-морфизм $F_{\mathrm{Sg}}^{2} \rightarrow G$.

ДокАЗАТЕЛЬСТво. Зафиксируем конечный главный $\boldsymbol{\Lambda}_{0}$-конус

$$
G=\left(V, X_{1}, \ldots, X_{n}, Y_{1}, \ldots, Y_{n}, f_{0}, f_{1}, f_{2}\right)
$$

глубины $r$ с диагональным корнем $v_{0}$. Положим $N:=|V|, m:=3(2 N)^{r}-2$. Пусть $G_{1}:=\left(V, X_{1}, \ldots, X_{n}\right) \uparrow u_{0}$. По лемме 7.6 , построим $m$-кратный р-морфизм $g_{0}: F \rightarrow G_{1}$, где $F=\left(U, \sqsubset_{1}, \ldots, \sqsubset_{n}\right)$ - конечное стандартное $n$-дерево глубины $r$ (см. определения $4.9,5.1$ ).

Определим конечную выпрямляющую игру $\operatorname{FRG}(G)$ с двумя игроками А и Е. Позиции в игре $\operatorname{FRG}(G)$ перед очередным ходом игрока А называются по-прежнему сетями. Каждая сеть - это отображение $h: H \longrightarrow V$, где $H \subseteq U^{2}$, удовлетворяющее следующим условиям:

(i) (устойчивость) $H$ замкнуто относительно предшествования:

$$
u \in H \& \exists i\left(v \sqsubset_{i 1} u \vee v \sqsubset_{i 2} u\right) \Longrightarrow v \in H,
$$

(ii) (слабая симметричность и гомоморфность по $\sigma_{0}$ )

$$
u \in H \Longrightarrow \sigma_{0}(u) \in H \& h\left(\sigma_{0}(u)\right)=f_{0}(h(u)),
$$


(iii) (сохранение диагонали)

$$
u \in H \& \sigma_{1}(u)=u \Longrightarrow h(u)=f_{1}(h(u)),
$$

(iv) (монотонность по горизонтальным стрелкам)

$$
u \in H \& v \in H \& u \sqsubset_{i 1} v \Longrightarrow h(u) X_{i} h(v) .
$$

Началъная позиция в $\operatorname{FRG}(G)$ - это сеть $h_{0}: H_{0} \longrightarrow V$, в которой

$$
\begin{aligned}
H_{0} & :=(U \times\{\lambda\}) \cup(\{\lambda\} \times U), \\
h_{0}(x, \lambda) & :=g_{0}(x), \quad h_{0}(\lambda, x):=f_{0}\left(g_{0}(x)\right) .
\end{aligned}
$$

Это определение корректно, так как $g_{0}(\lambda)=v_{0}$ - диагональная точка, и потому $f_{0}\left(v_{0}\right)=v_{0}$.

Каждая партия в $\operatorname{FRG}(G)$ состоит из конечного числа ходов и разбивается на этаnы, которые индексируются парами $(i, j)$, где $1 \leqslant j \leqslant i \leqslant r$. Пары (и этапы) упорядочены лексикографически:

$$
(i, j)<\left(i^{\prime}, j^{\prime}\right) \Longleftrightarrow j<j^{\prime} \vee j=j^{\prime} \& i<i^{\prime} .
$$

Множество $U^{2}$ разбивается на секторь $\Delta_{i j}$, где $0 \leqslant i, j \leqslant r$ :

$$
\Delta_{i j}:=\left\{(\alpha, \beta) \in U^{2}|| \alpha|=i,| \beta \mid=j\right\} .
$$

(В отличие от доказательства леммы 6.14, секторы нумеруются не "вглубь", а "ввысь", поэтому и обозначаются иначе.) Сектор $\Delta_{00}$ состоит из одной точки $(\lambda, \lambda)$, так как $F$ - стандартное дерево.

Опишем теперь правила игры. Игрок $\mathbf{A}$ начинает игру из позиции $h_{0}$. Если $h_{k}: H_{k} \rightarrow V$ - позиция перед очередным ходом $\mathbf{A}$ и партия находится на этапе $(i, j)$, то $\mathbf{A}$ имеет право своим ходом выбрать точку $u_{k} \in \Delta_{i j}-H_{k}$ одновременно с симметричной точкой $\sigma_{0}\left(u_{k}\right)$. Игрок $\mathbf{E}$ должен ответным ходом продолжить сеть $h_{k}$ на множество

$$
H_{k+1}:=H_{k} \cup\left\{u_{k}, \sigma_{0}\left(u_{k}\right)\right\}
$$

с соблюдением условий (i)-(iv). Если при этом оказывается, что $\Delta_{i j} \subseteq H_{k+1}$, то этап $(i, j)$ заканчивается, и начинается следующий этап - но только при условии $(i, j) \neq(r, r)$. Когда заканчивается этап $(r, r)$, партия завершается.

Точки $u_{k}, \sigma_{0}\left(u_{k}\right)$, выбранные $(k+1)$-м ходом игрока $\mathbf{A}$, будем называть запросами. Если они совпадают, то $u_{k}$ называется диагональным запросом.

Теоретически допустима ситуация, когда какой-то из игроков не может сделать очередного хода - в этом случае он считается проигравшим. Но как мы сейчас увидим, в данной игре это невозможно.

В нормально завершенной партии после заключительного хода игрока $\mathbf{E}$ возникает сеть $h: H \longrightarrow V$. Если она является р-морфизмом $h: F_{\mathrm{Sg}}^{2} \rightarrow G$, то выигрывает $\mathbf{E}$, в противном случае выигрывает $\mathbf{A}$.

Лемма 7.8. Всякая партия в игре $\operatorname{FRG}(G)$ нормалъно завериается.

ДокАзАтельство. Сначала заметим, что все секторы $\Delta_{i j}$ непусты. Действительно, $d_{F}=d_{G_{1}}=r$, поэтому $U$ содержит кортежи любой длины $\leqslant r$. Любая пара $(\alpha, \beta)$, где $|\alpha|=i,|\beta|=j$, попадает в сектор $\Delta_{i j}$. 
Из правил игры тогда следует, что А всегда может сделать свой ход: если этап $(i, j)$ не закончен, то остается неиспользованная точка в секторе $\Delta_{i j}$ и $\mathbf{A}$ может ее выбрать (в самом начале этапа годится любая точка этого сектора).

Теперь покажем, что $\mathbf{E}$ всегда может сделать ответный ход.

В начале партии сеть определена на всех секторах вида $\Delta_{0 i}$ и $\Delta_{i 0}$. Согласно правилам игры, после окончания этапа $(i, j)$ сеть определена на сек$\operatorname{topax} \Delta_{i j}$ и $\Delta_{j i}=\sigma_{0}\left[\Delta_{i j}\right]$. Поэтому на этапе $(i, j)$ сеть определена на всех секторах $\Delta_{i^{\prime} j^{\prime}}, \Delta_{j^{\prime} i^{\prime}}$, где $\left(i^{\prime}, j^{\prime}\right)<(i, j)$.

Предположим, что $\mathbf{A}$ на каком-то этапе $(i, j)$ делает свой $(k+1)$-й ход из позиции $h_{k}: H_{k} \longrightarrow V$, выбирая $(\alpha, \beta) \in \Delta_{i j},(\beta, \alpha) \in \Delta_{j i}$. Тогда предшественники $(\alpha, \beta)$ лежат в более ранних секторах. Действительно, $\left(\alpha^{-}, \beta\right) \in \Delta_{(i-1) j}$, $\left(\alpha, \beta^{-}\right) \in \Delta_{i(j-1)}$, причем при $j<i$ имеем $(i-1, j)<(i, j),(i, j-1)<(i, j)$ и $j-1<i, j \leqslant i-1$. При $i=j$ также $j-1<i$, a $\Delta_{(i-1) j}=\Delta_{(i-1) i}=\sigma_{0}\left[\Delta_{i(i-1)}\right]$. Поэтому в любом случае $\left(\alpha^{-}, \beta\right),\left(\alpha, \beta^{-}\right) \in H_{k}$. По той же причине (или в силу устойчивости) $\left(\alpha^{-}, \beta^{-}\right) \in H_{k}$. Если $\alpha^{-} \sqsubset_{s} \alpha, \beta^{-} \sqsubset_{t} \beta$, то, по монотонности сети $h_{k}$, получаем $v X_{s} v_{1}, v Y_{t} v_{2}$ для $v=h_{k}\left(\alpha^{-}, \beta^{-}\right), v_{1}=h_{k}\left(\alpha, \beta^{-}\right)$, $v_{2}=h_{k}\left(\alpha^{-}, \beta\right)$. Тогда, по свойству конвергенции для $G$, найдется $w \in V$, для которого $v_{1} Y_{t} w, v_{2} X_{s} w$. Выбрав произвольное такое $w$, определим

$$
h_{k+1}(\alpha, \beta):=w, \quad h_{k+1}(\beta, \alpha):=f_{0}(w),
$$

см. рис. 16. Этим обеспечено свойство (ii) для $h_{k+1}$.

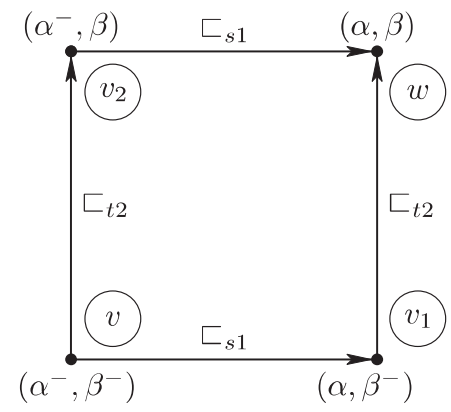

Рис. 16

Так мы действуем, если $\alpha \neq \beta$. В случае же $\alpha=\beta$ полагаем $h_{k+1}(\alpha, \alpha):=$ $w:=f_{1}\left(v_{2}\right)$, обеспечивая свойство (iii). При этом $s=t$ и по-прежнему $v_{1} Y_{t} w$, $v_{2} X_{s} w$. Действительно, тогда $v=f_{1}(v)=f_{1}\left(v_{1}\right)-$ в силу свойств (iii), (iv) для $h_{k}$ и $(\operatorname{Sg} 7)$ для $G, v_{2}=f_{0}\left(v_{1}\right)$ - в силу свойства (ii) для $h_{k}$. Так как $v X_{s} v_{1}$, по $(\mathrm{Sg} 6)$ для $G$ получаем $v_{1} Y_{s} w$, откуда, по $(\mathrm{Sg} 5)$, получаем $v_{2} X_{s} w$.

Устойчивость $H_{k+1}$ очевидна, так как предшественники всех новых точек лежат в $H_{k}$.

Монотонность следует из построения и свойства $(\mathrm{Sg} 5)$ для $G$. Действительно, новые стрелки в $H_{k+1}-$ это

$$
\left(\alpha^{-}, \beta\right) \sqsubset_{s 1}(\alpha, \beta), \quad\left(\alpha, \beta^{-}\right) \sqsubset_{t 2}(\alpha, \beta), \quad\left(\beta, \alpha^{-}\right) \sqsubset_{s 2}(\beta, \alpha), \quad\left(\beta^{-}, \alpha\right) \sqsubset_{t 1}(\beta, \alpha) .
$$

Отображение $h_{k+1}$ переводит их соответственно в

$$
v_{2} X_{s} w, \quad v_{1} Y_{t} w, \quad f_{0}\left(v_{2}\right) Y_{s} f_{0}(w), \quad f_{0}\left(v_{1}\right) X_{t} f_{0}(w) .
$$

Лемма доказана. 
Лемма 7.9. В игре $\operatorname{FRG}(G)$ имеется выигрышная стратегия для $\mathbf{E}$.

ДокАзАтЕльство. Согласно правилам игры, в любой завершенной партии заключительная сеть определена во всех секторах, т. е. является отображением $h: F_{\mathrm{Sg}}^{2} \longrightarrow G$. Оно монотонно по горизонтальным стрелкам и гомоморфно по $\sigma_{0}$.

Легко также видеть, что $h$ гомоморфно по $\sigma_{1}$, т. е.

$$
h\left(\sigma_{1}(\alpha, \beta)\right)=h(\beta, \beta)=f_{1}(h(\alpha, \beta)) .
$$

Действительно, в дереве $F$ имеем $\lambda \preceq \alpha$, поэтому в $F_{\mathrm{Sg}}^{2}$ есть последовательность горизонтальных стрелок из $(\lambda, \beta)$ в $(\alpha, \beta)$. По монотонности $h$, в $G$ тогда найдется последовательность $X$-стрелок (с соответствующими индексами) из $h(\lambda, \beta)$ в $h(\alpha, \beta)$. Благодаря $(\operatorname{Sg} 7)$, имеем

$$
f_{1}(h(\lambda, \beta))=f_{1}(h(\alpha, \beta)) .
$$

Аналогично, из $\lambda \preceq \beta$ следует, что

$$
f_{1}(h(\lambda, \beta))=f_{1}(h(\beta, \beta)) .
$$

Но по свойству сети (iii), $f_{1}(h(\beta, \beta))=h(\beta, \beta)$, поэтому из $(2),(3)$ получаем (1).

Таким образом, необходимо найти стратегию игрока $\mathbf{E}$, которая обеспечит свойство поднятия горизонтальных стрелок для заключительной сети $h$. Дадим описание такой стратегии.

ОПРедЕлЕниЕ 7.10. Рассмотрим этап $(i, j)$ с начальной позицией $g$. Запросы $(\alpha, \beta),(\gamma, \delta)$ этого этапа называются родственными, если

1) $\left(\alpha^{-}, \beta^{-}\right)=\left(\gamma^{-}, \delta^{-}\right)$,

2) $g\left(\alpha^{-}, \beta\right)=g\left(\gamma^{-}, \delta\right) \& g\left(\alpha, \beta^{-}\right)=g\left(\gamma, \delta^{-}\right)$,

3) $\forall t\left(\alpha^{-} \sqsubset_{t} \alpha \Rightarrow \gamma^{-} \sqsubset_{t} \gamma\right)$,

4) $\forall t\left(\beta^{-} \sqsubset_{t} \beta \Rightarrow \delta^{-} \sqsubset_{t} \delta\right)$,

см. рис. 17 .

Это определение корректно, поскольку, как уже отмечалось, $g$ определено на предшественниках запросов. Очевидно, что каждое из перечисленных в нем условий задает отношение эквивалентности, поэтому "родство" является отношением эквивалентности. Также очевидно, что родственные запросы попадают в один сектор.

Из гомоморфности $g$ по $\sigma_{0}$ следует, что если $(\alpha, \beta),(\gamma, \delta)$ родственны, то и их симметричные запросы $(\beta, \alpha),(\delta, \gamma)$ родственны. Более того, симметрия $\sigma_{0}$ переводит класс родства в секторе $\Delta_{i j}$ в класс родства в секторе $\Delta_{j i}$.

Каждый запрос $(\alpha, \beta)$ (как пара в $F^{2}$ с проекциями, отличными от $\lambda$ ) однозначно задается своими горизонтальным и вертикальным предшественниками $\left(\alpha^{-}, \beta\right),\left(\alpha, \beta^{-}\right)$. (В терминологии игры $\mathrm{RG}(G)$ из раздела $4,(\alpha, \beta)$ можно представить как запрос конвергенции $\left(\left(\alpha^{-}, \beta^{-}\right),\left(\alpha^{-}, \beta\right),\left(\alpha, \beta^{-}\right)\right)$.)

Рассмотрим запрос $(\alpha, \beta) \in \Delta_{i j}$. Обозначим

$$
\begin{array}{lll}
a:=\left(\alpha^{-}, \beta^{-}\right), & c:=\left(\alpha^{-}, \beta\right), & b:=\left(\alpha, \beta^{-}\right), \\
x:=g(a), & y:=g(b), & z:=g(c) .
\end{array}
$$




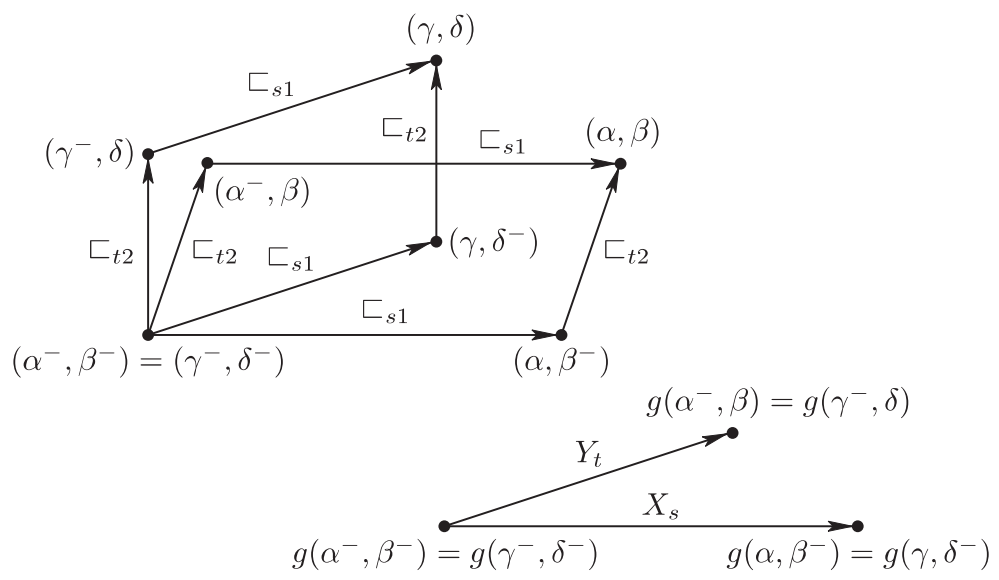

Рис. 17

Пусть

$$
\alpha^{-} \sqsubset_{s} \alpha, \quad \beta^{-} \sqsubset_{t} \beta,
$$

тогда

$$
x X_{s} y, \quad x Y_{t} z .
$$

Из определения ясно, что запросы $(\alpha, \beta),(\gamma, \delta)$ родственны, если и только если стрелка $a \sqsubset_{s 1}\left(\gamma, \delta^{-}\right)$- поднятие стрелки $x X_{s} y$ и $a \sqsubset_{t 2}\left(\gamma^{-}, \delta\right)$ - поднятие $x Y_{t} z$. Соответствующий класс родства обозначим через $C_{\text {ayzst }}$.

Таким образом, если $a \sqsubset_{s 1} b_{1}, \ldots, a \sqsubset_{s 1} b_{\mu_{1}}-$ все поднятия $x X_{s} y$, а $a \sqsubset_{t 2}$ $c_{1}, \ldots, a \sqsubset_{t 2} c_{\mu_{2}}$ - все поднятия $x Y_{t} z$, то запросы, родственные $(\alpha, \beta)$, соответствуют парам предшественников $\left(b_{p}, c_{q}\right)$, где $1 \leqslant p \leqslant \mu_{1}, 1 \leqslant q \leqslant \mu_{2}$. Запрос с предшественниками $b_{p}, c_{q}$ обозначим через $d_{p q}$. (Эта нумерация зависит от класса родства, но мы не вводим дополнительных индексов, чтобы не усложнять обозначения.)

Как говорилось выше, множество $X_{s}(z) \cap Y_{t}(y)$ непусто и любой его элемент годится в качестве ответа на любой запрос из данного класса. Симметричное к нему множество $Y_{s}\left(f_{0}(z)\right) \cap X_{t}\left(f_{0}(y)\right)$ состоит из возможных ответов на симметричные запросы. Пусть

$$
X_{s}(z) \cap Y_{t}(y)=\left\{w_{0}, \ldots, w_{l-1}\right\},
$$

см. рис. 18 .

Возможны три случая.

Случай I: $j<i$.

Тогда стратегия для $\mathbf{E}$ выбирает в качестве ответа на запрос $d_{p q}$ из данного класса точку $w_{[p+q]}$, где $[p+q]$ обозначает вычет $p+q$ по модулю $l$. Симметричный запрос $\sigma_{0}\left(d_{p q}\right)$ попадает в другой сектор, и ответом на него служит $f_{0}\left(w_{[p+q]}\right)$.

Случай II: $i=j$, но при этом запросы $(\alpha, \beta),(\beta, \alpha)$ не родственны. Иначе говоря, класс родства $(\alpha, \beta)$ не совпадает со своим симметричным классом.

Тогда стратегия почти не меняется. Пара запросов $(\alpha, \beta),(\beta, \alpha)$ составляет некоторый ход игрока А. Из них выбираем основной запрос по следующему 

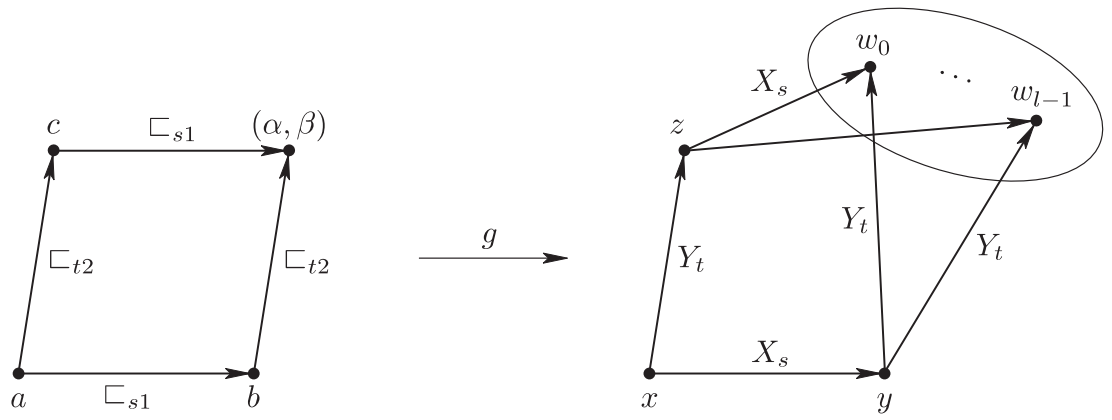

Рис. 18

правилу. Если на предыдущих ходах уже появлялись родственные им запросы, то основной запрос выбирается из того же класса родства, что и ранее. Если же родственных запросов раньше не было, то основным будем считать $(\alpha, \beta)$, если $\alpha<\beta$ (в некотором фиксированном линейном порядке на $F$ - например, лексикографическом) и $(\beta, \alpha)$ - в противном случае. Таким образом, класс родства основного запроса всегда состоит из основных запросов. Если $(\alpha, \beta)-$ первый по ходу игры основной запрос в своем классе, то для него составляем нумерации множества $X_{s}(z) \cap Y_{t}(y)$, множеств поднятий в точке $\left(\alpha^{-}, \beta^{-}\right)$стрелок $x X_{s} y$ и $x Y_{t} z$, а затем отвечаем на основные запросы из этого класса, как в случае I (и соответственно - на симметричные запросы).

Случай III: запросы $(\alpha, \beta),(\beta, \alpha)$ родственны. Согласно определению 7.10, это означает:

$$
i=j \& \alpha^{-}=\beta^{-} \& f_{0}(y)=z \& s=t .
$$

Действительно, $g\left(\alpha^{-}, \beta\right)=z, g\left(\beta^{-}, \alpha\right)=f_{0}\left(g\left(\alpha, \beta^{-}\right)\right)=f_{0}(y)$.

Такие запросы назовем почти диагональными.

В этом случае приходится изменить стратегию. Особняком стоят диагональные запросы, в которых $\alpha=\beta$. Как объяснялось раньше, ответы на них находятся однозначно (с помощью $f_{1}$ ). Для остальных почти диагональных запросов, как и в первых двух случаях, мы составляем список всевозможных ответов $w_{0}, \ldots, w_{l-1}$. Ответ на основной запрос $d_{p q}$ по-прежнему выбирается KaK $w_{[p+q]}$.

Основные запросы выбираются следующим образом.

Заметим, что если $a \sqsubset_{s 1} b_{1}, \ldots, a \sqsubset_{s 1} b_{\mu}-$ все поднятия $x X_{s} y$, то $a \sqsubset_{s 2}$ $c_{1}, \ldots, a \sqsubset_{s 2} c_{\mu}$ - все поднятия $x Y_{s} z$, где $c_{p}=\sigma_{0}\left(b_{p}\right)$ (в силу $(\operatorname{Sg} 5)$ и так как в данном случае $\left.a=\sigma_{0}(a), x=f_{0}(x), z=f_{0}(y)\right)$. Пусть $[p-q]$ - вычет $(p-q)$ по модулю $\mu$. Основным запросом будем считать $d_{p q}$, если $0<[p-q]<\mu / 2$, и $d_{q p}-$ если $0<[q-p]<\mu / 2$ (т. е. $\mu / 2<[p-q]<\mu$ ). В силу $(\operatorname{Sg} 5)$ для $F^{2}$, из $b_{p} Y_{s} d_{p q}$ и $c_{q} X_{s} d_{p q}$ получаем $c_{p} X_{s} \sigma_{0}\left(d_{p q}\right), b_{q} Y_{s} \sigma_{0}\left(d_{p q}\right)$. Таким образом, $\sigma_{0}\left(d_{p q}\right)=$ $d_{q p}$. Поэтому в паре симметричных запросов только один может оказаться основным. Кроме того, заметим, что $\sigma_{0}\left(d_{p p}\right)=d_{p p}$, т. е. при $p=q$ запрос $d_{p q}-$ диагональный.

Имеются еще пары запросов $d_{p q}, d_{q p}$, в которых $[p-q]=\mu / 2$ (при четном $\left.\mu\right)$; тогда и $[q-p]=\mu / 2$. Для них основной запрос не задается, и ответ игрока $\mathbf{E}$ 
выбирается произвольно (как уже говорилось, $\mathbf{E}$ может ответить на любой ход $\mathbf{A})$.

Остается проверить, что предложенная стратегия - выигрышная, т. е. обеспечивает поднятие горизонтальных стрелок. В действительности мы докажем более сильное утверждение.

Рассмотрим последовательность

$$
N_{j}:=3(2 N)^{r-j} \quad \text { при } 0 \leqslant j \leqslant r .
$$

Очевидно, что $N_{j} \geqslant 3$.

Будем говорить, что точка $a \in \Delta_{i j}-$ подходящая для сети $g: H \longrightarrow V$, если $g$ имеет $\left(N_{j}-2\right)$-кратное свойство поднятия горизонтальных стрелок в $a$.

В начальной (а следовательно, и в любой) позиции точки секторов вида $\Delta_{i 0}-$ подходящие. Это верно по построению $F$ и так как $m=N_{0}-2$.

Далее рассуждаем по индукции. Для сети $g: H \longrightarrow V$ точку из $H$ будем называть глубокой, если все горизонтально достижимые из нее точки $F^{2}$ попадают в $H$. Теперь индукцией по номеру этапа в лексикографической нумерации докажем, что в позиции в конце любого этапа все глубокие точки - подходящие.

Так как доказательство ведется по индукции, то надо рассматривать только новые глубокие точки, возникшие на данном этапе. Иными словами, для позиции $g$ в конце этапа $(i, j)$ надо доказать $\left(N_{j}-2\right)$-кратное свойство поднятия горизонтальных стрелок в точках сектора $\Delta_{(i-1) j}$ и $\left(N_{i}-2\right)$-кратное свойство поднятия горизонтальных стрелок в точках сектора $\Delta_{(j-1) i}$; ввиду симметрии, последнее равносильно $\left(N_{i}-2\right)$-кратному свойству поднятия вертикальных стрелок в точках сектора $\Delta_{i(j-1)}$.

Случай 1: $j<i$. Пусть

$$
c \in \Delta_{(i-1) j}, \quad a \sqsubset_{t 2} c, \quad g(a)=x, \quad g(c)=z, \quad z X_{s} w .
$$

Тогда $x Y_{t} z$, и, в силу коммутативности $G$, найдется $y$ такой, что $x X_{s} y Y_{t} w$. При этом $a \in \Delta_{(i-1)(j-1)}$ - глубокая точка, и, по предположению индукции, $g$ имеет в ней $\left(N_{j-1}-2\right)$-кратное свойство поднятия горизонтальных стрелок и $\left(N_{i-1}-2\right)$-кратное свойство поднятия вертикальных стрелок.

Пусть

$$
a \sqsubset_{s 1} b_{1}, \ldots, a \sqsubset_{s 1} b_{\mu_{1}}
$$

- все поднятия $x X_{s} y$ в $a$,

$$
a \sqsubset_{t 2} c_{1}, \ldots, a \sqsubset_{t 2} c_{\mu_{2}}
$$

- все поднятия $x Y_{t} z$, причем $c=c_{q}$. Тогда $\mu_{1} \geqslant N_{j-1}-2$.

Пусть $X_{s}(z) \cap Y_{t}(y)=\left\{w_{0}, \ldots, w_{l-1}\right\}$, причем $w=w_{\nu}$. Заметим, что $l \leqslant$ $N-2$. Действительно, $G$ - конечной глубины, поэтому $X_{s}, Y_{t}$ иррефлексивны и $z, y \notin X_{s}(z) \cap Y_{t}(y)$. Если $y \neq z$, то отсюда уже следует, что $l \leqslant N-2$. Если же $y=z$, то из иррефлексивности получаем $x \neq y$; кроме того, $x \notin$ $X_{s}(z) \cap Y_{t}(y)$, так как иначе бы мы имели $x X_{s} y=z X_{s} x$, что невозможно в шкале конечной глубины. Таким образом, в любом случае по крайней мере две точки не попадают в $\left\{w_{0}, \ldots, w_{l-1}\right\}$.

Рассмотрим сравнение относительно $p$

$$
[p+q]=\nu,
$$


или

$$
p \equiv \nu-q \quad(\bmod l) .
$$

Очевидно, что оно имеет решение на любом целочисленном отрезке длины $l$. Поэтому оно имеет по крайней мере

$$
\left[\frac{\mu_{1}}{l}\right] \geqslant\left[\frac{N_{j-1}-2}{N-2}\right]=\left[\frac{2 N N_{j}-2}{N-2}\right] \geqslant 2 N_{j} \geqslant N_{j}-2
$$

решений на множестве $\left\{1, \ldots, \mu_{1}\right\}$. По построению стратегии, для всех таких $p$ имеем $g\left(d_{p q}\right)=w$, и, следовательно, $c \sqsubset_{s 1} d_{p q}-$ поднятие $z X_{s} w$.

$\left(N_{i}-2\right)$-кратное свойство поднятия для вертикальных стрелок в секторе $\Delta_{(j-1) i}$ доказывается аналогично.

Случай 2: $i=j$. Доказываем свойство поднятия для горизонтальных стрелок. Как и в случае 1 , для данных $a, c, z, w$ находим $y$. Имеются два подслучая.

2.1. Класс родства $C_{a y z s t}$ (в предположении, что $\left.a \sqsubset_{t 2} c \& z X_{s} w\right)$ не содержит почти диагональных запросов. Это происходит, если $a \neq \sigma_{0}(a)$ или $f_{0}(y) \neq z$ или $s \neq t$. При выборе стратегии это соответствует случаю II. Здесь, в свою очередь, возникают два подслучая.

2.1.1. $C_{\text {ayzst }}$ состоит из основных запросов. Тогда доказательство дословно повторяет случай 1.

2.1.2. $C_{a y z s t}$ не содержит основных запросов. Тогда симметричный ему класс $C_{\sigma_{0}(a) f_{0}(z) f_{0}(y) t s}$ состоит из основных запросов и доказательство, аналогичное 2.1.1 (и случаю 1 ), устанавливает $N_{i}$-кратное свойство поднятия вертикальных стрелок в $\sigma_{0}(c)$. Отсюда, по симметрии, следует то же свойство для горизонтальных стрелок в $c$.

2.2. $C_{\text {ayzst }}$ состоит из почти диагональных запросов. Это означает, что

$$
a=\sigma_{0}(a) \& f_{0}(y)=z \& s=t,
$$

и при выборе стратегии возникает случай III.

Пусть

$$
a \sqsubset_{s 1} b_{1}, \ldots, a \sqsubset_{s 1} b_{\mu}
$$

- все поднятия $x X_{s} y$. Тогда

$$
a \sqsubset_{s 2} c_{1}, \ldots, a \sqsubset_{s 2} c_{\mu}
$$

- все поднятия $x Y_{t} z$, где $c_{p}=\sigma_{0}\left(b_{p}\right)$ для всех $p$. По предположению индукции, $\mu \geqslant N_{i-1}-2$.

Пусть $c=c_{p}, w=w_{\nu}$. Нам надо найти достаточное количество основных запросов этапа $(i, i)$, для которых стратегия выбирает ответ $w$. Это заведомо происходит, если выполнены условия

$$
0<[p-q]<\frac{\mu}{2} \&[p+q]=\nu,
$$

так как в этом случае запрос $d_{p q}-$ не диагональный и основной, а потому $g\left(d_{p q}\right)=w_{[p+q]}=w_{\nu}=w$. Таким образом, надо оценить число решений (относительно $p$ ) сравнения

$$
p \equiv \nu-q \quad(\bmod l)
$$

при условии $0<[p-q]<\mu / 2$. Для этого рассмотрим два подслучая. 
2.2.1. $1 \leqslant q<1+\mu / 2$. Тогда

$$
0<[p-q]<\frac{\mu}{2} \Longleftrightarrow q+1 \leqslant p<q+\frac{\mu}{2} .
$$

Это задает целочисленный отрезок длины не менее $[\mu / 2]-1$.

Число решений сравнения (\#) на отрезке длины $[\mu / 2]-$ не менее $[\mu /(2 l)]$. Поэтому на нашем отрезке - их не менее

$$
\left[\frac{\mu}{2 l}\right]-1 \geqslant\left[\frac{N_{i-1}-2}{2(N-2)}\right]-1=\left[\frac{2 N N_{i}-2}{2(N-2)}\right]-1=\left[\frac{N N_{i}-1}{N-2}\right]-1 \geqslant N_{i}-1 .
$$

2.2.2. $1+\mu / 2 \leqslant q \leqslant \mu$. Тогда

$$
0<[p-q]<\frac{\mu}{2} \Longleftrightarrow q+1 \leqslant p \leqslant \mu \vee 1 \leqslant p<q-\frac{\mu}{2},
$$

т. е. получаем дизъюнктное объединение отрезка и интервала. Число решений для $(\#)$ на отрезке - не менее $[(\mu-q-1) / l]$, а на интервале - не менее $[(q-$ $\mu / 2-1) / l]-1$. Напомним теперь свойство действительных чисел:

$$
\left[\varepsilon_{1}\right]+\left[\varepsilon_{2}\right] \geqslant\left[\varepsilon_{1}+\varepsilon_{2}\right]-1
$$

- оно сразу следует из

$$
\varepsilon_{1}<m_{1}+1 \& \varepsilon_{2}<m_{2}+1 \Longrightarrow \varepsilon_{1}+\varepsilon_{2}<m_{1}+m_{2}+2 .
$$

Поэтому общее число решений сравнения (\#) - не менее

$$
\left[\frac{(\mu-q-1)+(q-\mu / 2-1)}{l}\right]-2=\left[\frac{\mu / 2-2}{l}\right]-2 \geqslant N_{i}-2,
$$

так как

$$
\frac{\mu / 2-2}{l}=\frac{\mu-4}{2 l} \geqslant \frac{N_{i-1}-6}{2(N-2)}=\frac{N N_{i}-3}{N-2} \geqslant N_{i} .
$$

Итак, в случае 2.2 сравнение (\#) имеет по крайней мере $\left(N_{i}-2\right)$ решения, откуда следует нужное свойство поднятия горизонтальных стрелок.

Для поднятия вертикальных стрелок решается то же сравнение относительно $q$ при фиксированном $p$; все рассуждения аналогичны.

Это завершает доказательство свойства поднятия для $h$.

Лемма 7.9 доказана.

Основная лемма 7.7 сразу следует из лемм 7.8 и 7.9 .

\section{8. Погружение в классическую логику предикатов}

В работе [6] был определен "квадратный перевод”, погружающий квадраты модальных логик в классическую логику предикатов. Этот перевод естественно продолжается на квадраты Сегерберга.

Рассмотрим языки 1-го порядка (с равенством) $\mathscr{L} 1_{n}$ с 2-местными предикатными символами $R_{1}, \ldots, R_{n}$ и $\mathscr{L} 1_{n}^{2}$ - его обогащение счетным множеством других 2-местных предикатных символов $P_{1}, P_{2}, \ldots$ 
ОПредЕЛЕНИЕ 8.1. Пусть $A-(2 n+3)$-модальная формула, $x_{1}, x_{2}$ - различные индивидные переменные. Определим по индукции квадратный перевод $A^{2}\left(x_{1}, x_{2}\right) \in \mathscr{L} 1_{n}^{2}$

$$
\begin{aligned}
& \left(p_{i}\right)^{2}\left(x_{1}, x_{2}\right):=P_{i}\left(x_{1}, x_{2}\right), \\
& (\perp)^{2}\left(x_{1}, x_{2}\right):=\perp, \\
& (A \supset B)^{2}\left(x_{1}, x_{2}\right):=A^{2}\left(x_{1}, x_{2}\right) \supset B^{2}\left(x_{1}, x_{2}\right), \\
& \left(\boxminus_{i} A\right)^{2}\left(x_{1}, x_{2}\right):=\forall y\left(R_{i}\left(x_{1}, y\right) \supset A^{2}\left(y, x_{2}\right)\right), \\
& \left(\square_{i} A\right)^{2}\left(x_{1}, x_{2}\right):=\forall y\left(R_{i}\left(x_{2}, y\right) \supset A^{2}\left(x_{1}, y\right)\right), \\
& (\bigcirc A)^{2}\left(x_{1}, x_{2}\right):=A^{2}\left(x_{2}, x_{1}\right), \\
& \left(\bigcirc_{1} A\right)^{2}\left(x_{1}, x_{2}\right):=A^{2}\left(x_{2}, x_{2}\right), \\
& \left(\bigcirc_{2} A\right)^{2}\left(x_{1}, x_{2}\right):=A^{2}\left(x_{1}, x_{1}\right),
\end{aligned}
$$

где $y$ - новая переменная, не входящая в $A^{2}\left(x_{1}, x_{2}\right)$.

Для класса $n$-шкал $\mathscr{C}$ рассмотрим классическую теорию 1 -го порядка $\mathscr{L} 1_{n}^{2}$ структур вида $\mu=\left(W, \rho_{1}, \ldots, \rho_{n},\left(\Pi_{l}\right)_{l \geqslant 1}\right)$, где $\left(W, \rho_{1}, \ldots, \rho_{n}\right) \in \mathscr{C}, \Pi_{l}$ - бинарные отношения на $W$. Обозначим эту теорию через $\mathrm{Th}^{2}(\mathscr{C})$. Через $\mathscr{C}_{\mathrm{Sg}}^{2}$ обозначим класс всех квадратов Сегерберга шкал из $\mathscr{C}$, и пусть $\mathrm{PC}^{2}$ обозначает классическое исчисление предикатов в сигнатуре $\mathscr{L} 1_{n}^{2}$, a $\mathrm{PC}_{\text {fin }}^{2}$ - его финитный вариант, т. е. множество всех формул $\mathscr{L} 1_{n}^{2}$, общезначимых в конечных областях.

ПРЕДЛОЖЕНИЕ 8.2. 1) Для любой $(2 n+3)$-модалъной формуль $A$

$$
A \in \mathbf{L}\left(\mathscr{C}_{\mathrm{Sg}}^{2}\right) \Longleftrightarrow \forall x_{1} \forall x_{2} A^{2}\left(x_{1}, x_{2}\right) \in \operatorname{Th}^{2}(\mathscr{C}) .
$$

2) В частности, если $\boldsymbol{\Lambda}$ - $n$-модальная логика, то для любой $(2 n+3)$-модальной формуль $A$

$$
\mathbf{\Lambda}_{\mathrm{Sg}}^{2} \vdash A \Longleftrightarrow \forall x_{1} \forall x_{2} A^{2}\left(x_{1}, x_{2}\right) \in \operatorname{Th}^{2}(\mathbf{V}(\boldsymbol{\Lambda})) .
$$

3) Если же $\boldsymbol{\Lambda}$ является $\Delta$-элементарной и $\mathbf{V}(\boldsymbol{\Lambda})=\operatorname{Mod}(\Theta)$, то

$$
\Lambda_{\mathrm{Sg}}^{2} \vdash A \Longleftrightarrow \Theta \vdash_{\mathrm{PC}^{2}} A^{2}
$$

ДокАЗАТЕЛЬСтво. Стандартно, по аналогии со следствием 4.4 работы [6]. Для доказательства 1) достаточно заметить, что для шкалы $F=\left(W, \rho_{1}, \ldots, \rho_{n}\right)$, имеется биекция между моделями Крипке вида $M=\left(F_{\mathrm{Sg}}^{2}, \theta\right)$ и $\mathscr{L} 1_{n}^{2}$-структурами вида $\mu=\left(F, \Pi_{1}, \Pi_{2}, \ldots\right)$. При этом

$$
M,(a, b) \vDash A \Longleftrightarrow \mu \vDash A^{2}(a, b)
$$

- это легко доказывается по индукции.

2) сразу следует из 1) и определения 3.2. Утверждение 3) получается из 2) по теореме Гёделя о полноте.

Предложение доказано.

TEOPEMA 8.3. 1) $\left(\mathbf{K}_{n}\right)_{\mathrm{Sg}}^{2} \vdash A \Longleftrightarrow \vdash_{\mathrm{PC}^{2}} A^{2}$.

2) $\vdash_{\mathrm{PC}^{2}} A^{2} \Longleftrightarrow A^{2} \in \mathrm{PC}_{\text {fin }}^{2}$. 
ДокАзАтЕльство. 1) следует из утверждения 3) предложения 8.2.

2) $(\Rightarrow)$ очевидно.

$(\Leftarrow)$. Пусть $\nvdash_{\mathrm{PC}^{2}} A^{2}$, и пусть $\mathscr{C}-$ класс всех конечных $n$-шкал. Тогда $\left(\mathbf{K}_{n}\right)_{\mathrm{Sg}}^{2} \nvdash A-$ по 1). Но $\left(\mathbf{K}_{n}\right)_{\mathrm{Sg}}^{2}=\mathbf{L}\left(\mathscr{C}_{\mathrm{Sg}}^{2}\right)$ - по теореме 7.2. Поэтому $A \notin \mathbf{L}\left(\mathscr{C}_{\mathrm{Sg}}^{2}\right)$, откуда $\forall x_{1} \forall x_{2} A^{2}\left(x_{1}, x_{2}\right) \notin \mathrm{Th}^{2}(\mathscr{C})$ - по утверждению 1) предложения 8.2. Следовательно, $A^{2} \notin \mathrm{PC}_{\text {fin }}^{2}$.

Теорема доказана.

\section{9. Заключение}

Таким образом, в работе построен новый разрешимый и финитно аппроксимируемый фрагмент исчисления 2-местных предикатов с релятивизованными кванторами. Обратим внимание на то, что этот фрагмент - без равенства. При добавлении равенства картина меняется: как показано в [16], соответствующая 2 -мерная модальная логика $\mathbf{K}_{\delta}^{2}\left(\mathbf{K}^{2}\right.$ с выделенной диагональю) не является конечно аксиоматизируемой, а недавний результат С. П. Кикотя и А. Куруш устанавливает ее неразрешимость.

Вероятно, наш фрагмент можно еще расширить, разрешив использовать предикаты $R_{i}$ во всех атомарных формулах; в модальной логике это соответствует добавлению пропозициональных констант, которые интерпретируются как $R_{i}$. Подобная логика (с одной константой для $R$ ) предлагалась еще в работе Оквиста [14], но с тех пор продвижений в этом направлении не было, и вопросы о ее свойствах остаются открытыми.

Результаты настоящей работы, по-видимому, можно усилить и в другом направлении: как мы предполагаем, теоремы о финитной аппроксимируемости 6.16 и 7.2 распространяются на квадраты Сегерберга полимодальных логик с аксиомами рефлексивности и сериальности, а также временных логик.

Упомянем еще о связи между квадратами Сегерберга и алгебрами отношений. Как было по существу замечено в [15], модальные связки в этих логиках соответствуют операциям в алгебрах отношений, а общезначимые формулы превращаются в тождества в представимых алгебрах. В [6] показано, что в случае обычного квадрата минимальной логики эти тождества справедливы для всех алгебр отношений (благодаря теореме полноты), а для класса всех конечных алгебр не добавляется новых тождеств (благодаря финитной аппроксимируемости). Аналогичные следствия, скорее всего, можно получить и из результатов нашей работы.

Автор благодарит И. Б. Шапировского за проверку первоначального варианта рукописи и полезные замечания.

\section{Список литературы}

[1] K. Fine, "Logics containing K4. Part I", J. Symbolic Logic, 39 (1974), 31-42.

[2] В. Б. Шехтман, “Двумерные модальные логики”, Матем. заметки, 23:5 (1978), 759-772; англ. пер.: V. B. Shekhtman, "Two-dimensional modal logic", Math. Notes, 23:5 (1978), 417-424.

[3] V.B. Shehtman, "On some two-dimensional modal logics", Logic, methodology, and philosophy of science, v. 1, Abstracts of the 8th International Congress, Moscow, 1987, 326-330. 
[4] D. M. Gabbay, A. Kurucz, F. Wolter, M. Zakharyaschev, Many-dimensional modal logics: theory and applications, Stud. Logic Found. Math., 148, North-Holland Publishing Co., Amsterdam, 2003, xviii+747 pp.

[5] D. M. Gabbay, V. B. Shehtman, "Products of modal logics. Part 1", Log. J. IGPL, 6:1 (1998), 73-146.

[6] D. M. Gabbay, V. B. Shehtman, "Products of modal logics. Part 2: Relativised quantifiers in classical logic", Log. J. IGPL, 8:2 (2000), 165-210.

[7] D. Gabbay, V. Shehtman, "Products of modal logics. Part 3: Products of modal and temporal logics", Studia Logica, 72:2 (2002), 157-183.

[8] V. Shehtman, "Filtration via bisimulation", Advances in Modal Logic, 5, eds. R. Schmidt et al., King's Coll. Publ., London, 2005, 289-308.

[9] A. Kurucz, "Combining modal logics", Handbook of modal logic, Stud. Log. Pract. Reason., 3, Elsevier, Amsterdam, 2007, 869-927.

[10] D. Gabelaia, A. Kurucz, F. Wolter, M. Zakharyaschev, "Products of "transitive" modal logics", J. Symbolic Logic, 70:3 (2005), 993-1021.

[11] В. Б. Шехтман, "Неразрешимые исчисления высказываний", Вопросы кибернетики. Неклассические логики и их приложения, Наука, М., 1982, 74-116.

[12] A.V. Chagrov, V.B. Shehtman, "Algorithmic aspects of propositional tense logics", Computer science logic (Kazimierz, 1994), Lecture Notes in Comput. Sci., 933, Springer, Berlin, 1995, 442-455.

[13] K. Segerberg, "Two-dimensional modal logic", J. Philos. Logic, 2:1 (1973), 77-96.

[14] L. Aqvist, "A conjectured axiomatization of two-dimensional Reichenbachian tense logic", J. Philos. Logic, 8:1 (1979), 1-45.

[15] M. Marx, Y. Venema, Multi-dimensional modal logic, Appl. Log. Ser., 4, Kluwer Academic Publishers, Dordrecht, 1997, xiv+239 pp.

[16] С. П. Кикоть, "О квадратах модальных логик с выделенной диагональю”, Матем. заметки, 88:2 (2010), 261-274; англ. пер.: S. P. Kikot', "Axiomatization of modal logic squares with distinguished diagonal", Math. Notes, 88:2 (2010), 238-250.

[17] A. V. Chagrov, M.V. Zakharyaschev, Modal logic, Oxford Logic Guides, 35, The Clarendon Press, Oxford Univ. Press, New York, 1997, xvi+605 pp.

[18] J. Van Benthem, Modal logic and classical logic, Indices Monogr. Philos. Logic Formal Linguistics, III, Bibliopolis, Naples, 1985, 235 pp.

[19] P. Blackburn, M. de Rijke, Y. Venema, Modal logic, Cambridge Tracts Theoret. Comput. Sci., 53, Cambridge Univ. Press, Cambridge, 2001, xxii+554 pp.

[20] K. Segerberg, "On the logic of "to-morrow"”, Theoria, 33 (1967), 45-52.

[21] S. Kikot', "On modal definability of Horn formulas", Topology, algebra and categories in logic (TACL 2011), Abstracts of the 5th International Conference, University of Marseilles, Marseilles, 2011, 175-178.

B. Б. Шехтман (V.B. Shehtman)

Институт проблем передачи информации

им. А. А. Харкевича РАН;

Московский государственный университет

им. М. В. Ломоносова;

Национальный исследовательский университет

"Высшая школа экономики"

E-mail: shehtman@netscape.net
Поступила в редакцию 10.08.2011 